\title{
H-shaped miktobrush copolymer nanoassembly facilitates ultrafast nucleus delivery for multi- stimuli-cooperative tumour suppression
}

Huabing Chen ( $\nabla$ chenhb@suda.edu.cn )

Soochow University

Miya Zhang

Soochow University

Wenxue Dai

Soochow University

Tao Yang

Soochow University

Ting Li

Second Affiliated Hospital of Soochow University

\section{Tao Xu}

Second Affiliated Hospital of Soochow University

\section{Yibing Deng}

Jiangsu Key Laboratory of Translational Research and Therapy for Neuro-Psycho-Diseases

\section{Hong Yang}

Soochow University

Hengte Ke

Soochow University

Youliang Zhao

Soochow University

\section{Article}

Keywords: polymer nanoassembly, multi-stimuli responsiveness, nucleus delivery, triple negative breast cancer, photo-chemotherapy

Posted Date: February 4th, 2021

DOI: https://doi.org/10.21203/rs.3.rs-155902/v1

License: (a) (i) This work is licensed under a Creative Commons Attribution 4.0 International License.

Read Full License 



\section{H-shaped miktobrush copolymer nanoassembly facilitates}

2 ultrafast nucleus delivery for multi-stimuli-cooperative

3 tumour suppression

4 Miya Zhang ${ }^{1,2, \#}$, Wenxue Dai ${ }^{3, \#}$, Tao Yang ${ }^{1,2}$, Ting $\mathrm{Li}^{1,2}$, Tao $\mathrm{Xu}^{1,2}$, Yibin Deng ${ }^{1,2}$, Hong Yang ${ }^{1}$, 5 Hengte $\mathrm{Ke}^{1,2, *}$, Youliang Zhao ${ }^{3, *}$, and Huabing Chen ${ }^{1,2, *}$

$6{ }^{1}$ State Key Laboratory of Radiation Medicine and Protection, Soochow University, Suzhou 215123, 7 China

8 2Jiangsu Key Laboratory of Neuropsychiatric Diseases, and College of Pharmaceutical Sciences, 9 Soochow University, Suzhou 215123, China

$10{ }^{3}$ Suzhou Key Laboratory of Macromolecular Design and Precision Synthesis, Jiangsu Key 11 Laboratory of Advanced Functional Polymer Design and Application, State and Local Joint 12 Engineering Laboratory for Novel Functional Polymeric Materials, College of Chemistry, 13 Chemical Engineering and Materials Science, Soochow University, Suzhou 215123, China

16 Keywords: polymer nanoassembly, multi-stimuli responsiveness, nucleus delivery, triple-

17 negative breast cancer, photo-chemotherapy

18 \#These authors contributed equally to this work.

$19{ }^{*}$ Correspondence and requests for materials should be addressed to H.C. (chenhb@suda.edu.cn),

20 Y.Z. (ylzhao@suda.edu.cn), H.K. (htke@suda.edu.cn). 
22 Abstract: The potencies of antitumour compounds are often compromised by their restricted 23 subcellular delivery to nucleus, although a variety of smart vehicles have been extensively 24 designed to release drug in endocytic organelles. A major challenge remains in exploring the 25 nanocarriers with robust and spatiotemporal responsiveness for yielding efficient subcellular 26 targeting due to tumour heterogeneity. Herein, we show an H-shaped miktobrush copolymer 27 nanoassembly (NAs) with ultrafast nucleus delivery for multi-stimuli-cooperative suppression 28 against primary and metastatic triple-negative breast cancer (TNBC) models. The micellar NAs 29 display acidity- and glutathione-responsive drug releases through the protonation and disulfide30 bridge cleavage, which are further amplified by irreversible photo-controlled oxidation and phase 31 transition in a ratiometric manner, leading to rapid morphological destruction and ultrafast 32 cytoplasmic translocation into the nucleus from the lysosomes in a few minutes. These micellar 33 NAs thus show a distinctly enhanced cooperativity of photochemotherapeutic efficacy through 34 considerable apoptotic behavior, potently suppressing subcutaneous and orthotopic TNBC models, 35 together with enhanced survival rates. Moreover, these NAs yield preferable anti-metastatic 36 efficacy through the inhibition of metastasis-relevant proteins as compared to chemotherapy and 37 surgical resection. These results provide the insight into multi-stimuli-responsive polymers for 38 ultrafast nucleus delivery against aggressive tumours. 
40 Triple-negative breast cancers (TNBC) have been widely considered as a highly aggressive

41 subtype with distinct risk of metastasis and recurrence that accounts for the leading mortality

42 among females. Versatile therapeutic approaches have been extensively explored to yield efficient

43 treatments against TNBC including chemotherapy, phototherapy, and immunotherapy, thereby

44 arousing great interests in developing high-performance vehicles such as micelles and vesicles that

45 combine two or more modalities to achieve considerable antitumour treatments against $\mathrm{TNBC}^{1,2}$.

46 Reasonably, the precise delivery of vehicles incorporating antitumour cargoes to subcellular

47 targets is highly demanded to maximize antitumour efficacy with minimized adverse side effect

48 via enhanced tumour accumulation, deep penetration, intracellular drug release and accessibility

49 to subcellular target, reasonably leading to the widespread explorations of stimuli-responsive

50 nanoparticles for yielding potent antitumour efficacies ${ }^{3-7}$. For instance, various nanoparticles have

51 been rationally established to sense different endogenous stimuli such as mild acidity, enzyme,

52 glutathione and reactive oxygen species (ROS) that might promote intracellular drug release upon

53 endocytosis $^{8-13}$. However, these stimuli-responsive nanoparticles frequently suffer from multiple

54 intracellular barriers including endocytic recycling routes that induces drug efflux, enzyme- or

55 acidity-mediated degradation, and restricted drug/nanoparticle diffusion into cytoplasma, thereby

56 compromising their ability to deliver cargoes to access ultimate subcellular targets such as nucleus

57 and mitochondria. Therefore, it is highly urgent to explore an efficient approach to deliver

58 therapeutic cargoes to precisely reach subcellular target for yielding potent therapeutics against

59 TNBC models, together with reduced tumour metastasis and recurrence.

60 Various smart designs of nanoparticle vehicles have been explored to maximize their drug

61 delivery efficiencies including multiple-stimuli combination, hierarchically multistage-stimuli

62 response, and spatiotemporal control via exogenous stimuli such as light, temperature and 
63 ultrasound ${ }^{13-18}$, thus causing the interplay of a few responsive mechanisms such as hydrophobicity-

64 to-hydrophilicity conversion, charge conversion, chemical cleavage, and de-stabilization of weak

65 interactions (e.g. $\pi-\pi$ stacking and hydrogen bonding) in the interior of vehicles for preferable drug

66 release and enhanced accessibility to cytoplasma. In particular, exogenous stimuli might offer

67 selective and on-demanded release via precise manipulation on tumour site, while endogenous

68 stimuli are able to yield continuous drug release from the vehicles upon activation. However, the

69 expressions of endogenous stimuli molecules greatly vary in tumour cells including enzymes,

70 proteins, acidity and glutathione due to tumour heterogeneity, thus resulting in unsatisfied

71 exploration of robust and susceptive vehicles, and simultaneously the rational combination of

72 endogenous and exogenous stimuli also remains to be clarified for hurdling spatial barriers from

73 endocytic compartments to subcellular target ${ }^{14-22}$. Previously, we have explored light-responsive

74 nanoparticles that induce preferable drug release and efficient lysosomal rupture, thus promoting

75 considerable drug translocation into nucleus in $\sim 30$ min for enhanced antitumour efficacies against

$76 \mathrm{TNBC}^{14,15,23-30}$. We hypothesize that the rational combination of multi-stimuli-responsive

77 interplay and light-induced lysosome-cytoplasma-nucleus pathway might promote rapid nucleus

78 delivery for yielding potent therapy against TNBC with favorable prognosis. Here, we report a

79 multi-stimuli-responsive H-shaped miktobrush copolymer nanoassembly incorporating

80 photochemotherapeutic cargoes with ultrafast nucleus delivery for potent suppression against

81 primary and metastatic TNBC (Fig. 1). The micellar nanoassemblies (NAs) that were constructed

82 using brush-like copolymer as the scaffold displayed the acidity- and glutathione-responsive drug

83 releases that were further amplified by both photo-oxidation and photo-hyperthermia in a

84 ratiometric manner, subsequently leading to ultrafast lysosome-to-cytoplasm-nucleus

85 translocation in 5 min (Fig. 1a and 1b). Then, the micellar NAs showed a distinctly enhanced 
86 photochemotherapeutic efficacy through considerable apoptotic behavior, potently suppressing

87 subcutaneous and orthotopic TNBC models with enhanced survival rates, and simultaneously 88 generating considerable anti-metastatic efficacy as compared to chemotherapy and surgical 89 ablation through the inhibition of metastasis-relevant proteins.

91 Results

92 Synthesis, preparation and characterization.

93 To fabricate the multi-stimuli-responsive nanoassemblies, densely grafted brush-like copolymers

94 with two types of V-shaped heterografts were initially synthesized through a multi-step synthetic

95 route (Fig. 2a and Supplementary Fig. 1). The reductive-responsive disulfide group was introduced

96 from the initial chain transfer agent bis(2-hydroxyethyl)disulfide di(4-(benzodithioyl)-4-

97 cyanopentanoate) (S-CPDB), oxidative-responsive thioether groups were formed during thiol-

98 Michael addition reaction and thio-bromo click reaction, thermo-responsive $\operatorname{poly}(N-$

99 isopropylacrylamide) (PNIPAM) was grown via thiolactone-based aminolysis and telomerization,

100 and pH-sensitive poly(acrylic acid) (PAA) was obtained by consecutive controlled radical

101 polymerization and hydrolysis. In addition, biodegradable poly( $\varepsilon$-caprolactone) (PCL) was grown

102 from the hydroxyl-bearing Y junctions connecting with PNIPAM or poly(tert-butyl acrylate)

103 (PtBA) to form V-shaped heterografts.

104 Firstly, the reversible addition-fragmentation chain transfer (RAFT) copolymerization of 4105 vinylbenzyl-3-(2-bromo-2-methylpropanoyloxy)-2-hydroxymethyl-2-methylpropionate (VBHP)

106 and 2-maleimidyl-4-thiobutyrolactone (MTL) afforded disulfide-linked poly(VBHP-co-MTL) (S1)

107 containing hydroxyl, alkyl bromide and thiolactone functionalities. The number of monomer units

108 given by ${ }^{1} \mathrm{H}$ NMR analysis was similar $\left(\mathrm{DP}_{\mathrm{VBHP}} \approx \mathrm{DP}\right.$ MTL $\approx 14$, Supplementary Fig. 2 ), which was 
109 in good accordance with that expected from the alternating copolymerization system based on 110 styrene and maleimide. Secondly, disulfide-linked poly((St-PtBA)-co-MTL) (S2) was synthesized 111 by combination of grafting-from strategy and end-capping reaction. The atom transfer radical 112 polymerization (ATRP) of $t \mathrm{BA}$ using S1 macroinitiator gave the desired graft copolymer with $113 \mathrm{DP}_{\mathrm{P} t \mathrm{BA}}$ of 13.5 , followed by the thio-bromo click reaction to de-activate terminal bromide 114 functionality (Supplementary Fig. 3). Thirdly, the tandem amine-thiol-telomerization reactions 115 using S2, ethanolamine, 2,2'-azobis(isobutyronitrile) and NIPAM as the raw materials were 116 adopted to generate disulfide-linked poly((St-PtBA)-co-(MI-PNIPAM)) with DP PNIPAM of 25 (S3,

117 Supplementary Fig. 4). Finally, the ring-opening polymerization of CL using S3 macroinitiator 118 afforded three examples of disulfide-linked poly((St-PtBA/PCL)-co-(MI-PNIPAM/PCL)) 119 copolymers (S4-S6), followed by selective hydrolysis using trifluoroacetic acid catalyst to 120 generate more hydrophilic disulfide-linked poly((St-PAA/PCL)-co-(MI-PNIPAM/PCL)) 121 copolymers (H4-H6). In ${ }^{1} \mathrm{H}$ NMR spectroscopy (Fig. 2b), the characteristic signals of various 122 segments were noted at 4.06 ( $\mathrm{CH}_{2} \mathrm{O}$ of PCL), 3.99 ( $\mathrm{CHNH}$ of PNIPAM), 2.31 ( $\mathrm{CH}_{2} \mathrm{CO}$ of PCL), $1231.65\left(\mathrm{CH}_{2} \mathrm{CH}_{2} \mathrm{CH}_{2} \mathrm{O}\right.$ of PCL $), 1.44\left(\mathrm{CH}_{3}\right.$ of $\left.\mathrm{P} t \mathrm{BA}\right), 1.38\left(\mathrm{CH}_{2} \mathrm{CH}_{2} \mathrm{CH}_{2} \mathrm{O}\right.$ of $\left.\mathrm{PCL}\right)$ and $1.14 \mathrm{ppm}$ $124\left(\mathrm{CH}_{3}\right.$ of PNIPAM), and typical signals of $\mathrm{CH}_{2}$ connecting with disulfide or thioether $\left(\mathrm{CH}_{2} \mathrm{~S}\right)$ 125 appeared at 2.5-3.1 ppm. After hydrolysis, the typical signal of $\mathrm{P} t \mathrm{BA}$ at $1.44 \mathrm{ppm}$ disappeared 126 (Figure not shown). Based on ${ }^{1} \mathrm{H}$ NMR analysis, the polymerization degree of PCL grafts was 127 about 11 (S4, H4), 18 (S5, H5) and 35 (S6, H6), respectively. The successful synthesis of the 128 desired heterografted copolymers and their precursors were confirmed by ${ }^{1} \mathrm{H}$ NMR, GPC and FT129 IR spectra (Fig. 2b, Supplementary Fig. 2-6 and Table 1). The GPC traces of various copolymers 130 exhibited monomodal distribution (Supplementary Fig. 5). The apparent molar mass of S4-S6 $131 \quad\left(M_{\mathrm{n}, \mathrm{GPC}}=35.6-59.4 \mathrm{kDa}\right)$ was significantly lower than that given by ${ }^{1} \mathrm{H}$ NMR analysis $\left(M_{\mathrm{n}, \mathrm{NMR}}=\right.$ 
$132108-185 \mathrm{kDa}$ ), which could be ascribed to the reduced hydrodynamic volume of densely grafted

133 copolymers as compared to linear analogues originating from more compact structure.

134 The brush-like copolymer with reasonable lower critical transition temperature (LCST) of

$13539.4{ }^{\circ} \mathrm{C}$ (polymer H5, Fig. 2c) was mixed with indocyanine green (ICG) and doxorubicin (DOX)

136 in DMSO, and then the mixture was dispersed in water under ultrasonication, followed by the

137 formation of ICG/DOX-loaded micellar nanoassemblies (I/D-NAs) after the purification through

138 dialysis. The transmission electron microscopy (TEM) imaging shows that I/D-NAs with the

139 loading levels of $20 \%$ ICG and $10 \%$ DOX displayed the spherical morphology with the diameter

140 of $69.3 \pm 5.6 \mathrm{~nm}$ (Fig. 3a). Dynamic light scattering (DLS) shows that the nanoassembly had an

141 average hydrodynamic diameter of $84.6 \mathrm{~nm}$ with polydispersity index of 0.173 (Supplementary

142 Fig. 7), and also exhibited a good size stability during 7 days (Supplementary Fig. 8).

\section{Photothermal and photodynamic properties.}

145 The UV-vis absorption of I/D-NAs was observed and the result displayed a broadened peak at 818

$146 \mathrm{~nm}$, which was red-shifted from the original peak of ICG at $778 \mathrm{~nm}$ (Fig. 3b), implying the

147 formation of the $J$-type and $H$-type ICG aggregates with intermolecular $\pi-\pi$ stacking within the

148 nanoassemblies ${ }^{31-33}$. I/D-NAs showed an enhanced photostability in aqueous solution, reasonably

149 due to the encapsulation and interaction of the encapsulated cargoes within the nanocarriers

150 (Supplementary Fig. 9). To characterize the photoconversion properties of the I/D-NAs, its

151 photothermal conversion behavior was evaluated under $785 \mathrm{~nm}$ light exposure. It showed their 152 photothermal conversion efficiency $(\eta)$ of $24.6 \%$, which was higher than that $(\eta=20.3 \%)$ of free

153 ICG (Fig. 3c, Supplementary Fig. 10 and Table 2). Obviously, the nanoassembly displayed much 154 more temperature increase of $26.9^{\circ} \mathrm{C}$ within $5 \mathrm{~min}$ at the dose of $10.0 \mu \mathrm{g} \mathrm{mL} \mathrm{L}^{-1} \mathrm{ICG}$ as compared 
155 to free ICG $\left(\sim 15.4^{\circ} \mathrm{C}\right)$ and simultaneously exhibited a concentration-dependent improvement of 156 temperature (Supplementary Fig. 11). This result verifies that the I/D-NAs are able to maximize

157 the photothermal effect of photoactive agent that is highly favorable for hyperthermia-mediated 158 phototherapy, reasonably due to the enhanced non-radiative transition of encapsulated ICG 159 aggregates within the vehicles ${ }^{31-33}$.

160 The electron spin resonance (ESR) was further used to monitor the singlet oxygen generation 161 from I/D-NAs using 2,2,6,6-tetramethylpiperide (TEMP) as the spin-trapping agent of singlet 162 oxygen $^{34}$. The nanoassemblies exhibited the characteristic 1:1:1 multiplicity of TEMP-1-oxyl 163 under 5 min light irradiation, confirming the singlet oxygen generation (Supplementary Fig. 12).

164 Afterwards, its singlet oxygen quantum yield $\left(\Phi_{\Delta}\right)$ was further measured using 1,3-diphenyliso165 benzofuran (DPBF) as a reactive oxygen species (ROS) probe. Distinctly, I/D-NAs showed the 166 singlet oxygen quantum yield of 0.21 , while free ICG only had the quantum yield of 0.14 (Fig. 3c 167 and Supplementary Table 3). Meanwhile, these nanoassemblies effectively yielded the singlet 168 oxygen in a time-dependent manner under light exposure, while distinctly reduced singlet oxygen 169 generation was observed for free ICG (Supplementary Fig. 13). Hence, these nanoassemblies also 170 afford considerable singlet-to-triplet transition and subsequent energy transfer to molecular 171 oxygen for more singlet oxygen generation in addition to photothermal effect, potentially 172 facilitating the lysosomal rupture in tumour cells.

\section{Multi-stimuli-responsive drug release.}

175 The multi-stimuli-responsive drug release behavior of I/D-NAs was evaluated in response to acidic $176 \mathrm{pH}$, reduction and NIR-light-triggered thermal and ROS stimuli (Fig. 3d). The results showed that 177 I/D-NAs alone at physiological pH 7.4 exhibited a relatively slow and insufficient release profile 
178 of DOX compared to free ICG/DOX (Free I/D), confirming the reduced side effects from burst

179 release of chemotherapeutic drugs. Notably, I/D-NAs displayed the increased accumulative

180 releases of DOX at $\mathrm{pH} 5.0$, and was further enhanced by the addition of $10.0 \mathrm{mM}$ dithiothreitol

181 (DTT), reasonably being attributed to the deformation of PAA segments in acidic $\mathrm{pH}$ and disulfide

182 cleavage in reduction environment. Moreover, under light irradiation at $1.5 \mathrm{~W} \mathrm{~cm}^{-2}$ for $5 \mathrm{~min}$, the

183 accumulative release amount of DOX from I/D-NAs was significantly raised, reasonably due to

184 the deformation of PNIPAM and thioether segments caused by photo-hyperthermia and photo-

185 oxidation stimuli, as evidenced by the additionally increased DOX release by elevating the solution

186 temperature to $45^{\circ} \mathrm{C}$ (higher than LCST of $39.4^{\circ} \mathrm{C}$ ). Interestingly, the light-enhanced responsive

187 release profile could be simply optimized through a ratiometric manner (Supplementary Fig. 14),

188 indicating that the ratio of 2:1 between encapsulated ICG and DOX might trigger greater

189 dissociation of micellar assemblies, thus favoring rapid lysosomal escape and cytoplasmic

190 translocation of antitumour drugs. Thus, I/D-NAs loading ICG/DOX at the ratio of 2:1 were

191 selected for their following in vitro and in vivo assessments. The multi-stimuli responsiveness of

192 I/D-NAs was further confirmed by the morphological changes upon various stimuli

193 (Supplementary Fig. 15). The TEM images displayed the obvious deformation of I/D-NAs upon

194 exposure to acidic or reduction conditions, and the morphological destruction was also observed

195 for I/D-NAs under light irradiation. It confirms the superiority of I/D-NAs as a smart vehicle for 196 cancer therapeutics.

197

198 Cellular uptake and photo-induced lysosomal disruption.

199 To investigate the endocytic capacity of I/D-NAs, the cellar uptakes of DOX were assessed after

2006,12 , and $24 \mathrm{~h}$ incubation with $4 \mathrm{~T} 1$ tumour cells. The internalized amounts of DOX in tumour 
201 cells from I/D-NAs displayed the significantly improvement with time-dependency as compared

202 to Free I/D (Fig. 3e), indicating that these nanoassemblies possess a preferable capability to

203 improve the drug uptake. Subsequently, the cellular uptake pathway of I/D-NAs was demonstrated

204 in the presence of different endocytic inhibitors. I/D-NAs displayed a $28 \%$ decrease of DOX in

205 the cellular uptake upon treatment with chlorpromazine, indicating a clathrin-mediated energy-

206 dependent pathway of NAs (Fig. 3f). Afterward, the acridine orange (AO) staining was applied to

207 observe the destruction of lysosomes by ROS upon light irradiation. AO is an intracellular

208 indicator that emits red fluorescence in acidic lysosomes, and generates green fluorescence in

209 neutralized cytosol and nuclei. The lysosomes exhibited red fluorescence with PBS or irradiation

210 alone, indicating the intact lysosomes in the absence of I/D-NAs under irradiation or not. When

211 suffering from light irradiation, I/D-NAs at the dose of $2.0 \mu \mathrm{g} \mathrm{mL}^{-1}$ ICG resulted in obvious

212 lysosomal destruction, as evidenced by the disappearance of red fluorescence (Fig. 3g). In contrast,

213 free $\mathrm{I} / \mathrm{D}$ at the same concentration still displayed the yellow fluorescence, thus confirming

214 preferable intracellular ROS generation from I/D-NAs upon light exposure (Fig. 3g). Obviously,

215 I/D-NAs can effectively destruct lysosomal membranes under light irradiation, effectively

216 facilitating the cytoplasmic release of DOX in tumour cells.

218 Intracellular translocation from lysosomes to nucleus.

219 The confocal laser scanning microscopy (CLSM) was used to demonstrate the intracellular

220 distribution of I/D-NAs and photo-induced cytoplasmic translocation of antitumour compounds

221 (Fig. 3h). I/D-NAs exhibited high co-localization of 92.0\% in lysosomes without irradiation after

$2222 \mathrm{~h}$ incubation. However, after $5 \mathrm{~min}$ light irradiation at $1.5 \mathrm{~W} \mathrm{~cm}^{-2}$, the DOX/lysosome co-

223 localization rate significantly decreased to $23.0 \%$, and on the contrary, the red/blue co-localization 
224 rate was increased to $35.0 \%$, indicating that the light irradiation facilitates the fast lysosome-

225 cytoplasm-nucleus translocation of DOX at $5 \mathrm{~min}$ post-irradiation. Furthermore, the co-

226 localization rate of DOX with nucleus was increased to $85.0 \%$ after 15 min light exposure,

227 suggesting that the doxorubicin almost completely escaped from lysosomes to enter the nucleus

228 (Fig. 3i). The result shows that the rational combination of intrinsic acidic and reduced

229 environment in lysosomes, and NIR light-induced ROS and thermal stimuli, cooperatively

230 facilitates the deformation of I/D-NAs and lysosomal membrane rupture in a relatively short period

231 of time, resulting in considerable cytoplasmic translocation of DOX that further promotes the rapid

232 entry of DOX into the nucleus for subsequent DNA interaction to facilitate apoptosis ${ }^{23,35,36}$.

234 Synergistic cytotoxicity of photo-chemotherapy.

235 The photo-chemotherapeutic synergistic cytotoxicity was explored against 4T1 murine TNBC

236 tumour cells with I/D-NAs or Free I/D for $24 \mathrm{~h}$ under light exposure or not (Fig. 4a and

237 Supplementary Fig. 16). I/D-NAs resulted in a concentration-dependent cytotoxicity with $4.3 \mu \mathrm{g}$

$238 \mathrm{~mL}^{-1} \mathrm{IC}_{50(\mathrm{ICG})}$ without irradiation, while Free $\mathrm{I} / \mathrm{D}$ alone had relatively poor cytotoxicity $\left(\mathrm{IC}_{50}, 11.5\right.$

$239 \mu \mathrm{g} \mathrm{mL} \mathrm{L}^{-1} \mathrm{ICG}$ ), indicating the drug-loaded NAs could act as stimuli-responsive drug delivery

240 system to achieve enhanced chemotherapy via prominent cellular internalization and intrinsic

241 acidity- and reduction-responsive drug release. When exposed to 3 min light irradiation, I/D-NAs

242 showed an increased cytotoxicity with $2.2 \mu \mathrm{g} \mathrm{mL}^{-1} \mathrm{IC}_{50(\mathrm{ICG})}$, compared to Free I/D ( $\mathrm{IC}_{50} 8.7 \mu \mathrm{g}$

$243 \mathrm{~mL}^{-1} \mathrm{ICG}$ ), exhibiting a multi-stimuli-cooperative antitumour efficiency due to the synergistic

244 effect of light-induced photothermal/photodynamic cytotoxicity in addition to light-mediated

245 nucleus delivery of DOX. Moreover, the combination index $\left(\mathrm{CI}, \mathrm{CI}=\mathrm{D}_{\mathrm{A}} / \mathrm{IC}_{50(\mathrm{~A})}+\mathrm{D}_{\mathrm{B}} / \mathrm{IC}_{50(\mathrm{~B})}\right.$, in

246 which $\mathrm{D}_{\mathrm{A}}$ and $\mathrm{D}_{\mathrm{B}}$ represent the concentrations of $\mathrm{A}$ and $\mathrm{B}$ at $\mathrm{IC}_{50}$ in the presence of both $\mathrm{A}$ and $\mathrm{B}$; 
$247 \mathrm{IC}_{50(\mathrm{~A})}$ and $\mathrm{IC}_{50(\mathrm{~B})}$ represent the values of $\mathrm{IC}_{50}$ in the presence of $\mathrm{A}$ or $\mathrm{B}$ only, respectively ${ }^{37,38}$ ) was

248 calculated to assess the synergistic effect between chemotherapy and phototherapy of I/D-NAs 249 under light irradiation. The ICG-loaded nanoassemblies (ICG-NAs) with light irradiation were 250 employed to determine the phototherapy effect of ICG alone, and their $\mathrm{IC}_{50}$ with light irradiation

251 was measured to be $9.4 \mu \mathrm{g} \mathrm{mL}^{-1} \mathrm{ICG}$. The CI values of I/D-NAs at various molar ratios of 252 ICG:DOX were also calculated (Fig. 4b and Supplementary Fig. 17), indicating that I/D-NAs with 253 molar ratios of 2:1 and 3:1 exhibited distinctive synergistic cytotoxicity $(\mathrm{CI}<0.8)$ between 254 chemotherapy and phototherapy upon light irradiation, demonstrating a ratiometrically tunable 255 anticancer efficacy.

257 Cell apoptosis.

258 To investigate the cell damage mechanism of I/D-NAs, flow cytometry was utilized to detect 259 apoptotic levels using Annexin V-APC/DAPI staining. As shown in Fig. 4c and 4d, no obvious 260 apoptosis in tumour cells was observed for PBS with or without light irradiation, while Free I/D 261 exhibited $4.4 \%$ and $6.3 \%$ in early and late apoptosis under irradiation, respectively, indicating that 262 Free I/D can produce a mild apoptosis due to the combination of phototherapy and chemotherapy. 263 Whereas, the cells treated with ICG/DOX-loaded PEG-PCL micelles (I/D-Micelles) as a non264 responsive control exhibited a slight increased apoptotic effect at $15.3 \%$ and $12.7 \%$ for early and 265 late apoptosis in the absence of irradiation, respectively, and showed no significant increase of late 266 apoptosis upon light exposure. Importantly, I/D-NAs revealed a distinct improvement of apoptosis 267 in both early $(44.9 \%)$ and late $(25.4 \%)$ stage under irradiation via a prominent 268 photochemotherapeutic synergy, displaying a significant apoptotic increase as compared to I/D269 NAs without irradiation (22.7\% and $15.3 \%$ in early and late apoptosis, respectively). Besides, ICG- 
270 NAs as a control only facilitated the cell damage at $18.3 \%$ of early apoptosis and $7.6 \%$ of late

271 apoptosis. Hence, the potent apoptosis on tumour cells could be promoted through effective 272 synergistic photo-chemotherapy using multi-stimuli-responsive nanoassemblies.

273 In order to further assess the apoptotic mechanism of I/D-NAs, we examined the expression of 274 anti-apoptotic proteins and metastasis-relevant proteins in 4T1 cells with various treatments (Fig.

2754 4). The result displayed the lowest expressions of phosphorylated extracellular signal-regulated 276 kinase p-Erk1/2 (an anti-apoptotic protein that involves in tumour progression and metastasis in 277 the TNBC model $^{39}$ ) in tumour cells for I/D-NAs under light irradiation, while no obvious change 278 of p-Erk1/2 levels was found in other groups. Afterwards, the expression of Caspase-3 as a key 279 protein in execution-phase of cell apoptosis was then monitored, and the up-regulated expression 280 of Cl-Caspase-3 in 4T1 cells was observed for I/D-NAs under light irradiation, reasonably due to 281 their preferable photo-induced cytotoxicity as indicated in Fig. 4a. Furthermore, I/D-NAs also 282 resulted in the distinct decrease of $N$-Cadherin, a metastasis-relevant protein ${ }^{40}$, indicating that 283 cooperative photochemotherapy is potentially capable of regulating anti-apoptotic and metastasis284 relevant proteins for the inhibition of tumour progression and metastasis.

286 Pharmacokinetics and biodistribution.

287 The blood circulation of I/D-NAs was determined through the pharmacokinetic assessment (Fig. 288 5a). I/D-NAs exhibited a DOX elimination half-life $\left(t_{1 / 2 \beta}\right)$ of $5.2 \mathrm{~h}$, which is more than 4 times 289 longer when compared with that of Free I/D (1.2 h). Moreover, the $\mathrm{AUC}_{0 \sim \infty}$ value of I/D-NAs was 290 calculated to be 60.8, being a 3-fold increase as compared to Free I/D (Supplementary Table 3-4).

291 The targeting capability of I/D-NAs was further evaluated using ex vivo NIR fluorescent imaging 292 of the mice bearing 4T1 tumours. I/D-NAs displayed 4-fold increase of tumour accumulation of 
293 ICG at $24 \mathrm{~h}$ post-injection as compared to Free I/D, due to the effective EPR effect of the micellar

294 NAs (Fig. 5b and 5c). Then, the biodistribution behavior of I/D-NAs was evaluated, and showed

295 their highest distribution $\left(\sim 3.8 \% \mathrm{ID} \mathrm{g}^{-1}\right)$ of DOX in tumour at 24 hours after administration,

296 demonstrating their efficient targeting ability and reasonable time point for light irradiation (Fig.

297 5d and Supplementary Fig. 18). Distinctly, the preferable tumour accumulation and prolonged

298 circulation of I/D-NAs might facilitate prominent synergistic photo-chemotherapy against TNBC

299 tumours.

\section{Photo-induced hyperthermia and ROS.}

302 To investigate the in vivo photothermal effect of I/D-NAs at the tumour sites, the temperature

303 elevations in the tumour of the 4T1 tumour bearing mice under 5 min irradiation was recorded at

$30424 \mathrm{~h}$ post-injection. I/D-NAs raised the local temperature at tumour site by $\sim 15{ }^{\circ} \mathrm{C}$ under

305 irradiation ( $\left.1.5 \mathrm{~W} \mathrm{~cm}^{-2}, 7.5 \mathrm{mg} \mathrm{kg}^{-1} \mathrm{ICG}\right)$ and showed a dose-dependent temperature elevation

306 behavior, being preferable to the mild hyperthermia $\left(\Delta \mathrm{T}=8^{\circ} \mathrm{C}\right.$ ) induced by Free I/D (Fig. 5e and

307 5f, Supplementary Fig. 19-21). Hence, I/D-NAs facilitate the direct damage against the tumour

308 through photo-induced hyperthermia $\left(>45^{\circ} \mathrm{C}\right)$, which might trigger the deformation of micellar

309 NAs to promote the release and delivery of DOX. To demonstrate the in vivo photodynamic effect

310 of I/D-NAs, we also detected the generation of ROS in tumour using dihydroethidium (DHE)

311 staining, which can produce red fluorescence by transforming into 2-hydroxyehtidium. In Fig. 5f,

312 abundant ROS were generated from I/D-NAs in the tumour upon light irradiation, which were

313 more than 3-fold increase as compared to Free I/D (Supplementary Fig. 21). Thus, I/D-NAs

314 facilitate potent ROS generation for triggering oxidation-responsive release of DOX and

315 subsequent ultrafast lysosome-cytoplasma-nucleus delivery. 


\section{Antitumour efficacy on TNBC tumours.}

318 To elucidate the synergistic tumour suppressive efficacy, I/D-NAs, Free I/D, I/D-Micelles and

319 ICG-NAs were intravenously into the subcutaneous 4T1 TNBC tumour models at the dose of 7.5

$320 \mathrm{mg} \mathrm{kg}^{-1}$ ICG under 3 min irradiation or not, respectively. Subsequently, the tumour volume and

321 the survival rate were monitored during 60 days post-irradiation (Fig. $5 \mathrm{~g}$ and $5 \mathrm{~h}$ ). It shows that

322 PBS revealed the rapid tumour growth regardless of irradiation, suggesting that NIR light alone

323 have no impact on tumour growth. Free I/D and I/D-Micelles as the control exhibited a slight

324 tumour growth suppression and the survival time of mice was relatively short (less than 30 days)

325 as well, indicating that the synergistic photo-chemotherapeutic effect of ICG/DOX in a free form

326 and non-responsive micelles is not so potent to generate sufficient anticancer efficacy. ICG-NAs

327 with light exposure caused the tumour ablation for a short period of time, yet followed by the rapid

328 recurrence and regrowth of tumours. It shows that the phototherapy alone was not able to facilitate

329 sufficient and sustained tumour damage. Remarkably, I/D-NAs with light irradiation had the

330 prominent inhibition against tumour recurrence with prolonged survival time, revealing the

331 preferable antitumour efficacy achieved by potent synergistic photo-chemotherapy via the multi-

332 stimuli-responsive cooperativity of the nanoassemblies of rationally designed brush-like

333 copolymer. Taking advantage of intrinsic acidic and reduction environment in lysosomes, as well

334 as NIR light-induced ROS and thermal stimuli, the deformation of I/D-NAs and lysosomal

335 membrane rupture could be effectively utilized to undergo cytoplasmic translocation and ultrafast

336 nucleus delivery for causing prominent antitumour efficacy. Therefore, the light exposure of I/D-

337 NAs at tumour could not only trigger immediate and severe cell damage via photo-induced 
338 hyperthermia and ROS damage, but also facilitate the rapid lysosome-cytoplasm-nucleus delivery 339 of DOX to maximize photochemotherapeutic damage.

340 The hematoxylin and eosin (H\&E) staining suggests that I/D-NAs upon light irradiation resulted

341 in most severe cell injury with strong hemorrhagic inflammation at tumour due to the potent photo-

342 chemotherapy (Supplementary Fig. 22), while PBS as control showed no obvious tumour damage

343 regardless of light exposure. In addition, I/D-NAs had no remarkable damage on heart, liver,

344 spleen, lung and kidney of the tumour-bearing mice (Supplementary Fig. 23) with no significant

345 body weight loss (Supplementary Fig. 24), indicating that multi-stimuli-cooperative

346 nanoassemblies have a negligible side effect on the normal tissues, making them a promising

347 candidate for cancer therapy.

\section{Inhibition against primary TNBC tumour and metastatic nodules in lung.}

350 Inspired by the successful application of I/D-NAs against subcutaneous 4T1 models, we further 351 utilized I/D-NAs to treat highly aggressive orthotopic 4T1-Luc breast tumours that frequently 352 causes metastatic nodules in lungs. The tumour growth profile, bioluminescence images and 353 photos of harvested tumours were monitored during the experiment. PBS as a control exhibited 354 significant aggressive primary tumour growth regardless of irradiation or not (Fig. 6a-c). Free I/D

355 showed a slight inhibition of tumour growth even upon light irradiation, mainly due to rapid 356 elimination of free drugs in blood circulation. I/D-Micelles as non-responsive nanocarrier 357 exhibited the enhanced antitumour effects as compared to Free I/D, probably resulting from their 358 EPR effect. The surgery and ICG-NAs with irradiation were also found to cause temporary 359 ablation of tumours for a relative short period of time, but accompanied by obvious tumour 360 recurrence and regrowth at day 7 and day 13, respectively. Notably, total tumour ablation of the 
361 orthotopic breast tumour could be achieved by I/D-NAs under light irradiation, together without

362 any recurrence (Supplementary Fig. 25). Moreover, the observations of primary tumours using

363 H\&E staining, TUNEL, and Ki67 immunostaining showed that I/D-NAs with light exposure

364 caused the strongest destructive damage with less cell proliferation and more severe apoptotic level

365 (Supplementary Fig. 26), confirming their potent antitumour efficacy against primary tumours for

366 potentially preventing the survival and escape of tumour cells ${ }^{41}$.

367 Afterwards, the bioluminescence images of primary tumours, metastatic nodules in the lungs 368 and H\&E staining of lung section from the mice bearing orthotopic 4T1 tumours were further

369 observed. It shows that there was no significant bioluminescence from primary tumours and almost

370 no metastatic tumour nodules in the lungs for the mice treated with I/D-NAs under light irradiation

371 (Fig. 6d and 6e), indicating their significant anti-metastasis capability upon light exposure as

372 compared to surgical resection and ICG-NAs. The preferable inhibition of I/D-NAs against tumour

373 metastasis was in accordance with their capability to down-regulate the metastasis-related proteins

374 as indicated in Fig. 4e. In addition, no significant body weight loss was detected in the mice treated

375 with I/D-NAs upon light irradiation (Supplementary Fig. 27). Thus, I/D-NAs facilitate potent

376 cooperative photo-chemotherapeutic efficacy against orthotopic TNBC tumours and metastatic

377 nodules in the lungs through multi-stimuli-responsive drug release and ultrafast nucleus delivery.

379 Discussion

380 Endogenous stimuli are widely applied to trigger responsive drug release of smart vehicles for 381 improved chemotherapy ${ }^{42-45}$, and endogenously stimuli-responsive vehicles usually need to spend

382 several hours to accomplish the lysosome-cytoplasm-nucleus pathway ${ }^{46,47}$. In our design of $\mathrm{H}-$ 383 shaped miktobrush copolymer, there is only one disulfide bridge in a single polymer, which might 
384 consume less glutathione to be cleaved as compared to the conventional polymers with one

385 disulfide in each monomer, implying that the cleavage of $\mathrm{H}$-shaped structure is robust in response

386 to glutathione. In particular, this copolymer containing PAA and PNIPAM chains as hydrophilic

387 segments not only affords a prolonged circulation $\left(t_{1 / 2 \beta}, 5.2 \mathrm{~h}\right)$ as compared to that of Free $\mathrm{I} / \mathrm{D}\left(t_{1 / 2 \beta}\right.$,

$3881.2 \mathrm{~h}$ ), but also easily transits to be hydrophobic upon protonation and hyperthermia at tumour for

389 causing rapid phase transition of copolymer to release drug. However, the conventional

390 poly(ethylene glycol) (PEG) as an extensively used hydrophilic segment only provide a stealthy

391 function in blood, together without any other functionality for smart release. Moreover, the rational

392 combination of endogenous and exogenous stimuli not only amplified drug release of I/D-NAs,

393 but also effectively destructed spatial barriers in tumour cells such as the capture of endocytic

394 compartments and limited drug diffusion to subcellular target ${ }^{14-22}$. In our previous studies, light-

395 responsiveness facilitated efficient lysosomal rupture through photo-induced singlet oxygen and

396 hyperthermia, resulting in considerable drug delivery into nucleus in $\sim 30$ min for enhanced

397 antitumour efficacy ${ }^{14,15,23-30}$. These rationally designed NAs possess the multi-stimuli-responsive

398 cooperativity (I/D-NAs) that results in the ultrafast lysosome-cytoplasma-nucleus translocation in

399 about $\sim 5$ min for potent therapy against primary TNBC tumours and lung metastasis. Reasonably,

400 this design of H-shaped miktobrush copolymer nanoassembly provides a sophisticated multiple

401 functionalization approach for efficient nucleus delivery of antitumour compounds.

402 In summary, the H-shaped miktobrush copolymer nanoassemblies were successfully

403 constructed, resulting in potent suppression against primary and metastatic TNBC through multi-

404 stimuli-cooperative photo-chemotherapy caused by the combination of considerable smart drug

405 release and ultrafast lysosome-cytoplasma-nucleus delivery. The micellar nanoassemblies

406 exhibited acidity- and glutathione-responsive drug release of photochemotherapeutic cargos at a 
407 low consumption level of glutathione, which are further amplified by irreversible light-triggered 408 deformation in a ratiometric manner. These multi-stimuli-responsive design cooperatively leads to 409 the rapid phase transition from hydrophilic segments into hydrophobic chains and lysosomal 410 rupture, both of which account for the ultrafast delivery of cargo from the lysosomes into the 411 nucleus in $\sim 5 \mathrm{~min}$. The distinctly enhanced cooperativity of photochemotherapy was demonstrated

412 by considerable apoptotic behavior, potent suppression against tumour models, as well as

413 improved survival rates. Notably, these NAs also facilitated preferable anti-metastatic efficacy

414 through the inhibition of metastasis-relevant proteins as compared to chemotherapy and surgical

415 ablation. Our strategy provides a multi-stimuli-responsive nanoplatform for ultrafast nucleus 416 delivery in cancer therapy.

\section{Methods}

419 Preparation of I/D-NAs. To prepare I/D-NAs, $2.0 \mathrm{mg} \mathrm{ICG,} 1.0 \mathrm{mg}$ desalted DOX, and $10.0 \mathrm{mg}$ 420 polymer $\mathrm{H} 5$ were mixed in $200 \mu \mathrm{L}$ of DMSO, and then the mixed solution was dispersed into 2.0 $421 \mathrm{~mL}$ distilled water under ultrasonication $(15 \mathrm{~min})$. Subsequently, the solution of I/D-NAs were 422 obtained after purification via dialysis (cut-off $3.5 \mathrm{kDa}$ MW, $24 \mathrm{~h}$ ). All of the above procedures 423 were performed at $25^{\circ} \mathrm{C}$. According to similar procedures, $\mathrm{PEG}_{45}-b$ - $\mathrm{PCL}_{60}$ diblock copolymer was 424 employed to prepare the micelles without any stimuli-responsiveness as the control nanoparticles 425 (I/D-Micelles). The free ICG/DOX samples (Free I/D) were prepared by dissolving the drugs in 426 5\% DMSO aqueous solution.

428 Characterization. The morphology of I/D-NAs was observed using transmission electron 429 microscope (TEM, Tecnai-G20). The hydrodynamic diameters were measured using Zetasizer 
ZS90 (Malvern, U.K.) at $25^{\circ} \mathrm{C}$. The drug loading and entrapment efficiency of ICG and DOX

431 within micelles were measured by using ultrafiltration centrifuge (Cut-off $10 \mathrm{KDa} \mathrm{MW}$ ) at $4{ }^{\circ} \mathrm{C}$.

432 The absorption spectra of ICG were measured using a UV-vis spectrophotometer (UV2600, 433 Shimadzu) and the fluorescent spectra of DOX were measured using Fluorescence 434 Spectrophotometer (LS 55, PerkinElmer).

Drug release. The drug release behaviors of DOX from I/D-NAs and Free I/D were evaluated by 437 the dialysis method. Samples (1.0 mL each) were added in various solutions including $\mathrm{pH} 7.4$ and $438 \mathrm{pH} 5.0$ buffers in the presence of $10.0 \mathrm{mM}$ DTT or not. Then, the solutions were performed in air 439 with a contrast temperature oscillator shaker at $37^{\circ} \mathrm{C}$. The concentration of ICG and DOX were 440 measured at $0.5,1,2,4,8,12$ and $24 \mathrm{~h}$, respectively. For NIR-light-triggered drug release, the 441 amounts of DOX released from I/D-NAs were evaluated in $\mathrm{pH} 5.0$ buffers in the presence of 10 $442 \mathrm{mM}$ DTT after $785 \mathrm{~nm}$ irradiation $\left(1.5 \mathrm{~W} \mathrm{~cm}^{-2}, 3 \mathrm{~min}\right)$.

444 Photothermal effect and photothermal conversion efficiency. Free I/D and I/D-NAs (each 0.5 $445 \mathrm{~mL})$ at the concentration of $1.0,2.0,5.0,10.0,25.0$ and $50.0 \mu \mathrm{g} \mathrm{mL}^{-1}$ ICG were irradiated $(785$ $446 \mathrm{~nm}, 1.5 \mathrm{~W} \mathrm{~cm}^{-2}$ ) for $300 \mathrm{~s}$, and their temperature were monitored with a thermometer every $30 \mathrm{~s}$. 447 To calculate the photothermal conversion efficiency, $0.5 \mathrm{~mL}$ of Free I/D and I/D-NAs of $10.0 \mu \mathrm{g}$ $448 \mathrm{~mL}^{-1} \mathrm{ICG}$ were exposed to $785 \mathrm{~nm}$ laser at $1.5 \mathrm{~W} \mathrm{~cm}^{-2}$ for $5 \mathrm{~min}$, and then the laser were removed 449 to cool the solution down to room temperature, with the temperature of the solution recorded each $45030 \mathrm{~s}$. The photothermal conversion efficiency $(\eta)$ is calculated by the following equation:

$$
\eta=\frac{h A\left(T_{\max }-T_{a m b}\right)-Q_{0}}{I\left(1-10^{-A \lambda}\right)}
$$


where $h$ is the heat transfer coefficient, $A$ is the surface area of the container, $T_{\max }$ is the

453 maximum temperature during the process, $T_{a m b}$ is the surrounding ambient temperature, $Q_{0}$ is the 454 heat input rate due to light absorption of the solvent, $I$ is the laser power, and $A_{\lambda}$ is the absorbance 455 of the sample at an excitation wavelength of $785 \mathrm{~nm}$.

457 Quantum yield of singlet oxygen, ESR and fluorescence lifetime measurement. To measure 458 the singlet oxygen quantum yield $\left(\Phi_{\Delta}\right)$, I/D-NAs were evaluated with 1,3-diphenylbenzofuran 459 (DPBF) used as a probe, and indocyanine green (ICG) was used as a reference compound $\left(\Phi_{\Delta}{ }^{\text {ICG }}\right.$ $460=0.14)$. The solutions of ICG and I/D-NAs containing DPBF $(30.0 \mu \mathrm{M})$ were irradiated by 785 $461 \mathrm{~nm}$ laser at $1.5 \mathrm{~W} \mathrm{~cm}^{-2}$ for $300 \mathrm{~s}$, and the absorbance of DPBF at $417 \mathrm{~nm}$ were recorded. The 462 singlet oxygen quantum yield $\left(\Phi_{\Delta}\right)$ of I/D-NAs were calculated using the following equation $\Phi_{\Delta}=$ $463 \Phi_{\Delta}^{\mathrm{ICG}} \cdot W \cdot I^{\mathrm{ICG}} /\left(W^{\mathrm{ICG}} \cdot I\right)$, where $W$ and $W^{\mathrm{ICG}}$ are the photobleaching rates of DPBF in the presence 464 of I/D-NAs and ICG, respectively; $I$ and $I^{I C G}$ are the rates of light absorption by I/D-NAs and ICG, 465 respectively. The electron spin resonance (ESR) technique was employed to distinguish the type 466 of ROS. ROS from I/D-NAs were monitored by mixing $5.0 \mu \mathrm{L}$ 2,2,6,6-tetramethylpiperide 467 (TEMP), which was used as the spin-trapping agent of singlet oxygen $\left({ }^{1} \mathrm{O}_{2}\right)$, with $100 \mu \mathrm{L}$ I/D-NAs, 468 followed by $785 \mathrm{~nm}$ irradiation at $1.5 \mathrm{~W} \mathrm{~cm}^{-2}$ for $5 \mathrm{~min}$. The ESR spectra of I/D-NAs in the 469 presence of TEMP without irradiation was collected as a control. For fluorescence lifetime 470 measurement, the UV absorption spectra of I/D-NAs and Free I/D were measured using a UV-Vis 471 spectrophotometer (UV2600, Shimadzu), and the fluorescence spectrum were recorded by a 472 fluorescence spectrophotometer (PerkinElmer LS 55). Then, the fluorescence quantum yields were 473 calculated by the following equation: $\Phi_{F(\text { sample })}=\left(F_{\text {sample }} / F_{\text {ref }}\right)\left(A_{\text {ref }} / A_{\text {sample }}\right)\left(n_{\text {sample }}^{2} / n_{\text {ref }}^{2}\right) \Phi_{F(\text { ref })}$, in 474 which $F$ is the measured fluorescence (area under the emission peak), $A$ is the absorbance at the 
475 excitation position, $n$ is the refractive index of the solvent. ICG in water was used as the reference $476 \quad\left(\Phi_{F(r e f)}=0.005\right)$.

478 Photostability and chemical stability. To determine photostability, Free I/D and I/D-NAs (each

$4793.0 \mathrm{~mL})$ at the concentration of $5.0 \mu \mathrm{g} \mathrm{mL}^{-1} \mathrm{ICG}$ were irradiated $\left(785 \mathrm{~nm}, 1.5 \mathrm{~W} \mathrm{~cm}^{-2}\right)$ for $0.5,1$, $4802,3,5,7$ and 10 min, respectively, followed by the measurements of the absorbance of ICG using 481 a UV-vis spectrophotometer. To determine chemical stability, Free I/D and I/D-NAs (each 3.0 mL) 482 at the concentration of $10.0 \mu \mathrm{g} \mathrm{mL} \mathrm{m}^{-1}$ ICG were dispersed in various media including culture 483 medium, pH 7.4 buffer and pH 5.0 buffer, and then their absorbance and fluorescence spectra were 484 monitored at $0,4,8,12,24$ and $48 \mathrm{~h}$, respectively.

486 Cellular uptake and endocytic pathways. 4T1 tumour cells $\left(1.0 \times 10^{6} /\right.$ well $)$ were seeded on six487 well culture plates and incubated overnight in $37{ }^{\circ} \mathrm{C}$ with RPMI 1640 containing $10 \%$ FBS. Free $488 \mathrm{I} / \mathrm{D}$ and I/D-NAs were added into the wells for 6, 12 and $24 \mathrm{~h}$ incubation. ICG and DOX from the 489 cells were extracted using DMSO, and measured by UV-vis and fluorescent assay, respectively. 490 For endocytic pathway, PBS and different inhibitions $\left(10 \mu \mathrm{g} \mathrm{mL} L^{-1}\right.$ chlorpromazine, $100 \mu \mathrm{g} \mathrm{mL}^{-1}$ 491 amiloride, $50 \mu \mathrm{g} \mathrm{mL}-1$ nystatin) were added into the cells for $1 \mathrm{~h}$ incubation at $37^{\circ} \mathrm{C}$ or $4{ }^{\circ} \mathrm{C}$ in 492 RPMI-1640 medium, respectively. Next, Free I/D and I/D-NAs at the concentration of $10.0 \mu \mathrm{g}$ $493 \mathrm{~mL}^{-1}$ ICG were added into the plate for $2 \mathrm{~h}$ incubation. Then the ICG and DOX amounts extracted 494 from the cells were analyzed using UV-vis and a fluorescence spectrophotometer.

496 In vitro cytotoxicity and disruption of lysosomal membranes. For in vitro cytotoxicity 497 assessment, 4T1 cells $\left(5.0 \times 10^{3} /\right.$ well $)$ were treated with Free I/D and I/D-NAs at the various doses 
498 of $0.4,1.0,2.0,4.0,8.0,16.0$ and $20.0 \mu \mathrm{g} \mathrm{mL}-1$ ICG for $24 \mathrm{~h}$ incubation, followed by $3 \mathrm{~min}$ 499 irradiation $\left(785 \mathrm{~nm}, 1.5 \mathrm{~W} \mathrm{~cm}^{-2}\right)$ or not. Another $24 \mathrm{~h}$ later, the cell viability was evaluated using 500 MTT assay. To evaluate the disruption of lysosomal membranes, acridine orange (AO) was used 501 as an intracellular indicator of acidic lysosomes. The 4T1 cells $\left(5.0 \times 10^{4} /\right.$ well $)$ were treated with 502 PBS, Free I/D and I/D-NAs at the dose of $2.0 \mu \mathrm{g} \mathrm{mL}-1$ ICG for $6 \mathrm{~h}$ at $37^{\circ} \mathrm{C}$. Then, the cells were 503 washed with PBS and incubated in fresh medium, followed by 3 min irradiation at $785 \mathrm{~nm}(1.5 \mathrm{~W}$ $\left.504 \mathrm{~cm}^{-2}\right)$. After $1 \mathrm{~h}$, the 4T1 cells were washed and incubated with $\mathrm{AO}(6.0 \mu \mathrm{M}, 1.0 \mathrm{~mL})$ for $30 \mathrm{~min}$, 505 finally subjected to fluorescence microscopical observation.

507 Intracellular distribution. $4 \mathrm{~T} 1$ cells $\left(5.0 \times 10^{4}\right.$ cells/well $)$ in a glass bottom dish were treated with 508 I/D-NAs for $2 \mathrm{~h}$ at $37^{\circ} \mathrm{C}$, and then washed by PBS. For the sample without irradiation, $1.0 \mathrm{~mL}$ 509 Hoechst $33342\left(1.0 \mu \mathrm{g} \mathrm{mL}^{-1}\right)$ was added and incubated for another $10 \mathrm{~min}$ at $37^{\circ} \mathrm{C}$. Then, $1.0 \mathrm{~mL}$ 510 Lysotracker Green DND-26 (100 nM) was added and incubated for another $5 \mathrm{~min}$. For the sample

511 observed at 5 min post-irradiation, the cells were treated with $1.0 \mathrm{~mL}$ of Hoechst $33342(1.0 \mu \mathrm{g}$ $\left.512 \mathrm{~mL}^{-1}\right)$ for $10 \mathrm{~min}$ at $37^{\circ} \mathrm{C}$, and then illuminated for $3 \mathrm{~min}$ at $785 \mathrm{~nm}\left(1.5 \mathrm{~W} \mathrm{~cm}{ }^{-2}\right)$, followed by

513 incubation with $1.0 \mathrm{~mL}$ Lysotracker Green DND-26 (50 nM) for $5 \mathrm{~min}$. For the sample observed 514 at 15 min post-irradiation, the cells were firstly illuminated, and then incubated with Hoechst 51533342 and Lysotracker Green DND-26. Finally, the cells were observed using CLSM (Zeiss 516 LSM710).

518 Apoptosis. The mechanism of cell death was explored using Annexin V-APC Apoptosis Kit. The $5194 \mathrm{~T} 1$ cells $\left(1.0 \times 10^{5} /\right.$ well $)$ were cultured in a 12 -well plate and then treated with PBS, Free I/D, 520 I/D-Micelles, I/D-NAs, ICG-NAs at $20 \mu \mathrm{g} \mathrm{mL}{ }^{-1}$ ICG for $24 \mathrm{~h}$ incubation, followed by $3 \mathrm{~min} 785$ 
$521 \mathrm{~nm}$ irradiation at $1.5 \mathrm{~W} \mathrm{~cm}^{-2}$ or not. Another $6 \mathrm{~h}$ later, the cells were digested with trypsin without

522 EDTA and collected by centrifugation at $3000 \mathrm{rpm}$, and then washed twice using PBS. Afterwards,

523 the cells were stained with Annexin V-APC Apoptosis Kit and DAPI and further analysed by flow

524 cytometry (FC500, Beckman Coulter).

525

526 Western blotting. 4T1 cells $\left(1.0 \times 10^{6} /\right.$ well $)$ were cultured in six-well plates and then incubated 527 with PBS, Free I/D, I/D-Micelles I/D-NAs and ICG-NAs for $24 \mathrm{~h}$, followed by 3 min irradiation $528\left(785 \mathrm{~nm}, 1.5 \mathrm{~W} \mathrm{~cm}^{-2}\right)$ or not. Another $6 \mathrm{~h}$ later, cells were washed twice by PBS and then lysed on 529 ice with lysate for $1 \mathrm{~h}$. Subsequently, the proteins from the cells were extracted and the 530 concentrations of the proteins were measured by BCA protein assay (Thermo Fisher Scientific). 531 The proteins $(10.0 \mu \mathrm{g})$ for each group were separated by a 10\% SDS-polyacrylamide gel and then 532 transferred to a nitrocellulose membrane, blocked with 5\% skim milk in TBST for $1 \mathrm{~h}$. Then, the 533 membrane was incubated with anti-N-cadherin (CST), anti-Caspase-3 (CST), anti-Cl-Caspase-3 534 (CST), anti-p-Erk (CST), anti-Erk (Santa Cruz Biotech) and GAPDH (Affinity) overnight at $4{ }^{\circ} \mathrm{C}$, 535 washed with TBST five times, followed by incubation with secondary antibody (ZSGB-BIO) for $536 \quad 1.5 \mathrm{~h}$ and visualized by an ECL-plus detection system (GE healthcare).

538 Ex vivo NIRF imaging and biodistribution. The female BALB/c mice bearing 4T1 tumour were 539 constructed by subcutaneously injecting $4 \mathrm{~T} 1$ cells $\left(2.0 \times 10^{6}\right.$ cells/mouse $)$ into the flanks. Then, 540 the mice were injected intravenously with Free I/D and I/D-NAs at the doses of $7.5 \mathrm{mg} \mathrm{kg}^{-1} \mathrm{ICG}$ $541(\mathrm{n}=3)$. Then, the various tissues including heart, liver, spleen, lung, kidney and tumour were 542 extracted from the mice at $6,12,24,48 \mathrm{~h}$ post-injection. The ex vivo fluorescence imaging of 543 various tissues at $24 \mathrm{~h}$ post-injection was observed using IVIS Lumina II with the excitation 
544 wavelength of $745 \mathrm{~nm}$. For biodistribution, methanol was used to extract ICG and DOX from the

545 different tissues at various time. Finally, DOX and ICG amounts were determined using

546 fluorescence and UV spectrophotometer, respectively.

548 In vivo thermography. Free $\mathrm{I} / \mathrm{D}$ and $\mathrm{I} / \mathrm{D}-\mathrm{NAs}$ were injected intravenously into the female BALB/c

549 mice bearing 4T1 tumours at the doses of 2.5, 5.0, and $7.5 \mathrm{mg} \mathrm{kg}^{-1} \mathrm{ICG}$, respectively. Then, the

550 mice were irradiated for $3 \mathrm{~min}$ using a $785 \mathrm{~nm}$ laser $\left(1.5 \mathrm{~W} \mathrm{~cm}^{-2}\right)$ at $24 \mathrm{~h}$ post-injection, and an

551 infrared camera (FLIR E50) was used to monitor the temperature at the tumour region during the 552 irradiation.

554 In vivo antitumour efficacy. The female BALB/c mice bearing subcutaneous $4 \mathrm{~T} 1$ tumours were 555 injected with PBS, Free I/D, I/D-Micelles, I/D-NAs and ICG-NAs at the dose of $7.5 \mathrm{mg} \mathrm{kg}^{-1} \mathrm{ICG}$ 556 on day 0,2 , and 4 via tail vein. At $24 \mathrm{~h}$ post-injection, the tumours of each group were irradiated

557 with $785 \mathrm{~nm}$ laser at $1.5 \mathrm{~W} \mathrm{~cm}^{-2}$ for 3 min or not. The tumour growth was observed during the 558 following 30 days, then the volumes were measured using a vernier caliper and calculated by this 559 formula: $V=X \times Y^{2} / 2$, in which $X$ and $Y$ represent the length and width of the tumour, respectively. 560 The survival rates of these mice were further observed during 60 days post-irradiation.

562 Orthotopic triple-negative breast cancer (TNBC) model. The female BALB/c mice bearing 563 orthotopic 4T1 breast tumours were made by injecting luc-4T1 cells into the fat pad of breast, and 564 then were injected with PBS, Free I/D, I/D-Micelles, I/D-NAs and ICG-NAs via the tail vein on 565 day 0,2 , and 4, followed with $3 \mathrm{~min} 785 \mathrm{~nm}$ irradiation at $1.5 \mathrm{~W} \mathrm{~cm}^{-2}$ at the tumour site $24 \mathrm{~h}$ later. 566 For surgery group, the tumours were ablated on day 0 . Then, the tumour volumes were measured 
567 and calculated during the following 20 days. Moreover, the mice were injected with $200 \mu \mathrm{L}$ sodium

568 fluorescein solution at the concentration of $15.0 \mathrm{mg} \mathrm{mL}^{-1}$ on day $0,2,6,9,12,16,20$ and the

569 bioluminescence of mice was observed using IVIS Lumina II. On day 20, all the mice were

570 sacrificed to extract their tumours and lungs to image their ex vivo bioluminescence. In addition,

571 the tumours were weighted and the metastatic tumour nodules in the lungs were also counted.

572

573 H\&E, Ki67, and TUNEL staining. The BALB/c mice bearing subcutaneous 4T1 tumours were

574 intravenously injected with PBS, Free I/D, I/D-Micelles, I/D-NAs and ICG-NAs at the dose of 7.5

$575 \mathrm{mg} \mathrm{kg}^{-1} \mathrm{ICG}$, followed with $3 \mathrm{~min}$ irradiation $\left(785 \mathrm{~nm}, 1.5 \mathrm{~W} \mathrm{~cm}^{-2}\right)$ or not at $24 \mathrm{~h}$ post-irradiation.

576 Another $6 \mathrm{~h}$ later, various tissues including heart, liver, spleen, lung, spleen and tumour were

577 extracted and stored in the $4 \%$ formaldehyde solution. Afterwards, each tissues was cut into thin

578 sections for H\&E staining. The mice bearing orthotopic $4 \mathrm{~T} 1$ tumours were treated with the same

579 procedure as described above, then the tumours were extracted and cut into slices for Ki67 and

580 TUNEL staining. Lungs extracted from TNBC models were further cut into slices for H\&E

581 staining. Then, the sections for H\&E and Ki67 staining were performed using IX73 bright field

582 microscope (Olympus), while the TUNEL-stained sections were observed using a confocal laser

583 scanning microscope (Zeiss LSM650).

584

585 Statistical analysis. All data were presented as the mean value \pm SD. Statistical analysis was

586 performed using two-tailed Student's $t$ test or one-way ANOVA with Tukey's post hoc test.

587 Statistical significance was established at $p<0.05$, where ${ }^{*} p<0.05,{ }^{* *} p<0.01$, and ${ }^{* * *} p<0.001$. 
589 Data availability. The data that support the findings of this study are available within the paper

590 and its Supplementary Information files. Raw data are available from the corresponding authors 591 upon reasonable request.

592

593

594 


\section{References}

596 1. van der Meel, R., et al. Smart cancer nanomedicine. Nat. Nanotechnol. 14, 1007-1017 597 (2019).

598 2. Peer, D., et al. Nanocarriers as an emerging platform for cancer therapy. Nat. Nanotechnol. $5992,751-760$ (2007).

600 3. Murakami, M., et al. Improving drug potency and efficacy by nanocarrier-mediated 601 subcellular targeting. Sci. Transl. Med. 3, 64 ra62 (2011).

602 4. Wang, A.Z., Langer, R. \& Farokhzad, O.C. Nanoparticle delivery of cancer drugs. Annu. 603 Rev. Med. 63, 185-198 (2012).

604 5. Shi, J., Kantoff, P.W., Wooster, R. \& Farokhzad, O.C. Cancer nanomedicine: progress, 605 challenges and opportunities. Nat. Rev. Cancer 17, 20-37 (2017).

606 6. Chen, G., Roy, I., Yang, C. \& Prasad, P.N. Nanochemistry and nanomedicine for 607 nanoparticle-based diagnostics and therapy. Chem. Rev. 116, 2826-2885 (2016).

608 7. Sun, Q., Zhou, Z., Qiu, N. \& Shen, Y. Rational design of cancer nanomedicine: 609 nanoproperty integration and synchronization. Adv. Mater. 29, 1606628 (2017).

610 8. Zhang, Z., et al. Near infrared laser-induced targeted cancer therapy using 611 thermoresponsive polymer encapsulated gold nanorods. J. Am. Chem. Soc. 136, 612 7317-7326 (2014).

613 9. Chen, H., et al. Polyion complex vesicles for photoinduced intracellular delivery of 614 amphiphilic photosensitizer. J. Am. Chem. Soc. 136, 157-163 (2014).

615 10. de la Rica, R., Aili, D. \& Stevens, M.M. Enzyme-responsive nanoparticles for drug release 616 and diagnostics. Adv. Drug Deliv. Rev. 64, 967-978 (2012).

617 11. Chen, H., et al. Precise nanomedicine for intelligent therapy of cancer. Sci. China Chem. $618 \quad 61,1503-1552(2018)$.

619 12. Ge, Z. \& Liu, S. Functional block copolymer assemblies responsive to tumor and 620

622 13. Guo, Z., et al. pH-sensitive polymeric micelles assembled by stereocomplexation between 623 PLLA-b-PLys and PDLA-b-mPEG for drug delivery. J. Mater. Chem. B 7, 334-345 (2019). 
624 14. Shanmugam, V., Selvakumar, S. \& Yeh, C.S. Near-infrared light-responsive nanomaterials 625 in cancer therapeutics. Chem. Soc. Rev. 43, 6254-6287 (2014).

626 15. Zhang, Z., Wang, J. \& Chen, C. Near-infrared light-mediated nanoplatforms for cancer 627 thermo-chemotherapy and optical imaging. Adv. Mater. 25, 3869-3880 (2013).

628 16. Yang, G., Liu, J., Wu, Y., Feng, L. \& Liu, Z. Near-infrared-light responsive nanoscale drug delivery systems for cancer treatment. Coord. Chem. Rev. 320-321, 100-117 (2016).

630 17. Wang, J., et al. NIR-activated supersensitive drug release using nanoparticles with a flow 631 core. Adv. Funct. Mater. 26, 7516-7525 (2016).

632 18. Cao, J., et al. Near-infrared light-triggered micelles for fast controlled drug release in deep 633 tissue. Biomaterials 34, 6272-6283 (2013).

634 19. Luo, D., et al. Rapid light-triggered drug release in liposomes containing small amounts of unsaturated and porphyrin-phospholipids. Small 12, 3039-3047 (2016).

636 20. Luo, D., et al. Doxorubicin encapsulated in stealth liposomes conferred with light-triggered 637 drug release. Biomaterials 75, 193-202 (2016).

638 21. Meng, Z., et al. NIR-laser-switched in vivo smart nanocapsules for synergic photothermal 639 and chemotherapy of tumors. Adv. Mater. 28, 245-253 (2016).

640 22. Qian, C., et al. Light-activated hypoxia-responsive nanocarriers for enhanced anticancer 641 therapy. Adv. Mater. 28, 3313-3320 (2016).

642 23. Wang, Y., et al. Light-responsive nanoparticles for highly efficient cytoplasmic delivery 643 of anticancer agents. ACS Nano 11, 12134-12144 (2017).

644 24. An, X., et al. Rational Design of Multi-stimuli-responsive nanoparticles for precise cancer 645 therapy. ACS Nano 10, 5947-5958 (2016).

646 25. Yang, X., et al. Near-infrared light-triggered, targeted drug delivery to cancer cells by 647 aptamer gated nanovehicles. Adv. Mater. 24, 2890-2895 (2012).

648 26. Ma, Y., Huang, J., Song, S., Chen, H. \& Zhang, Z. Cancer-targeted nanotheranostics: 649 recent advances and perspectives. Small 12, 4936-4954 (2016).

650 27. Deng, Y., et al. Cyanine-anchored silica nanochannels for light-driven synergistic thermo651 chemotherapy. Small 13, 1602747 (2017).

652 28. Yang, H., et al. Micelles assembled with carbocyanine dyes for theranostic near-infrared 653 fluorescent cancer imaging and photothermal therapy. Biomaterials 34, 9124-9133 (2013). 
654 29. Wan, Z., et al. Highly efficient hierarchical micelles integrating photothermal therapy and singlet oxygen-synergized chemotherapy for cancer eradication. Theranostics 4, 399-411 (2014).

30. Wang, Y., et al. Smart albumin-biomineralized nanocomposites for multimodal imaging and photothermal tumor ablation. Adv. Mater. 27, 3874-3882 (2015).

31. Guo, Z., et al. Heavy-atom-modulated supramolecular assembly increases antitumor potency against malignant breast tumors via tunable cooperativity. Adv. Mater. n/a, 2004225 (2020).

32. He, H., et al. Photoconversion-tunable fluorophore vesicles for wavelength-dependent photoinduced cancer therapy. Adv. Mater. 29, 1606690 (2017).

33. Ye, S., et al. Rational design of conjugated photosensitizers with controllable

34. Yang, T., et al. Bifunctional tellurium nanodots for photo-induced synergistic cancer

35. Wang, C., et al. Enhancing cell nucleus accumulation and DNA cleavage activity of anticancer drug via graphene quantum dots. Sci. Rep. 3, 2852 (2013).

36. Tian, W., Xie. X. \& Cao, P. Magnoflorine improves sensitivity to doxorubicin (DOX) of breast cancer cells via inducing apoptosis and autophagy through AKT/mTOR and p38 signaling pathways. Biomed. Pharmacother. 121, 109139 (2020).

37. Chou, T. \& Talalay, P. Analysis of combined drug effects: a new look at a very old problem.

Trends Pharmacol. Sci. 4, 450-454 (1983).

38. Soriano, A.F., et al Synergistic effects of new chemopreventive agents and conventional cytotoxic agents against human lung cancer cell lines. Cancer Res. 59, 6178 (1999).

677 39. Liu, W., et al. Clinical significance of pAkt and pErk $1 / 2$ expression in early-stage breast cancer patients treated with anthracycline-based adjuvant chemotherapy. Oncol. Lett. 9,

680 40. Gayther, S.A., et al. Tagging single nucleotide polymorphisms in cell cycle control genes 681 and susceptibility to invasive epithelial ovarian cancer. Cancer Res. 67, 3027 (2007).

682 41. Yu, G., et al. A discrete organoplatinum(II) metallacage as a multimodality theranostic 683 platform for cancer photochemotherapy. Nat. Commun. 9, 4335 (2018). 
684 42. Du, J., Lane, L.A. \& Nie, S. Stimuli-responsive nanoparticles for targeting the tumor 685 microenvironment. J. Control. Release 219, 205-214 (2015).

686 43. Li, R. \& Xie, Y. Nanodrug delivery systems for targeting the endogenous tumor 687 microenvironment and simultaneously overcoming multidrug resistance properties. $J$. $688 \quad$ Control. Release 251, 49-67 (2017).

689 44. Li, Z., Song, N. \& Yang, Y. Stimuli-responsive drug-delivery systems based on $690 \quad$ supramolecular nanovalves. Matter 1, 345-368 (2019).

691 45. Peng, M. Stimuli-responsive nanocarriers for drug delivery, tumor imaging, therapy and 692 theranostics. Theranostics 10, 4557-4588 (2020).

693 46. Li, Q., et al. AIEgen-functionalized mesoporous silica gated by cyclodextrin-modified CuS 694 for cell imaging and chemo-photothermal cancer therapy. ACS Appl. Mater. Interfaces 10, 695 12155-12163 (2018).

696 47. Xue, Y., et al. Enhanced targeted delivery of doxorubicin based on acid induced charge 697 reversal and combinational stimuli-responsive nanocarrier. Adv. Eng. Mater. 20, 1701151 698 (2018). 
701 M. Z. and W. D. contributed equally to this work. This work was supported by National Key R\&D

702 Program of China (2020YFA0710700), National Natural Science Foundation of China (51873143, 703 21971181, 32071373, and 81871480), Key Research \& Development Program of Jiangsu Province

704 (BE2020763) and Priority Academic Program Development of Jiangsu Higher Education 705 Institutions (PAPD). All animal experiment protocols were approved by the Animal Care and Use 706 Committee of Soochow University, and complied with all relevant ethical regulation.

\section{Author contributions}

709 H.C., Y.Z. and H.K. conceived the idea and supervised the project. M.Z., W.D., T.Y., and T.X.

710 performed the experiments. M.Z., W.D., H.K. and H.C. contributed to the scheme and figures.

711 H.K., Y.Z. and H.C. contributed to the revision of original draft. M.Z., T.L., Y.D., H.Y., Y.Z. and

712 H.C. performed the analysis of all the data. H.K., Y.Z. and H.C. prepared the manuscript. All the

713 authors discussed the results and commented on the manuscript.

\section{Competing interests}

716 The authors declare no competing financial interests.

718 Additional information

719 Supplementary information is available for this paper.

720 Reprints and permissions information is available online at www.nature.com/reprints.

721 Correspondence and requests for materials should be addressed to H.C., Y.Z., H.K. 

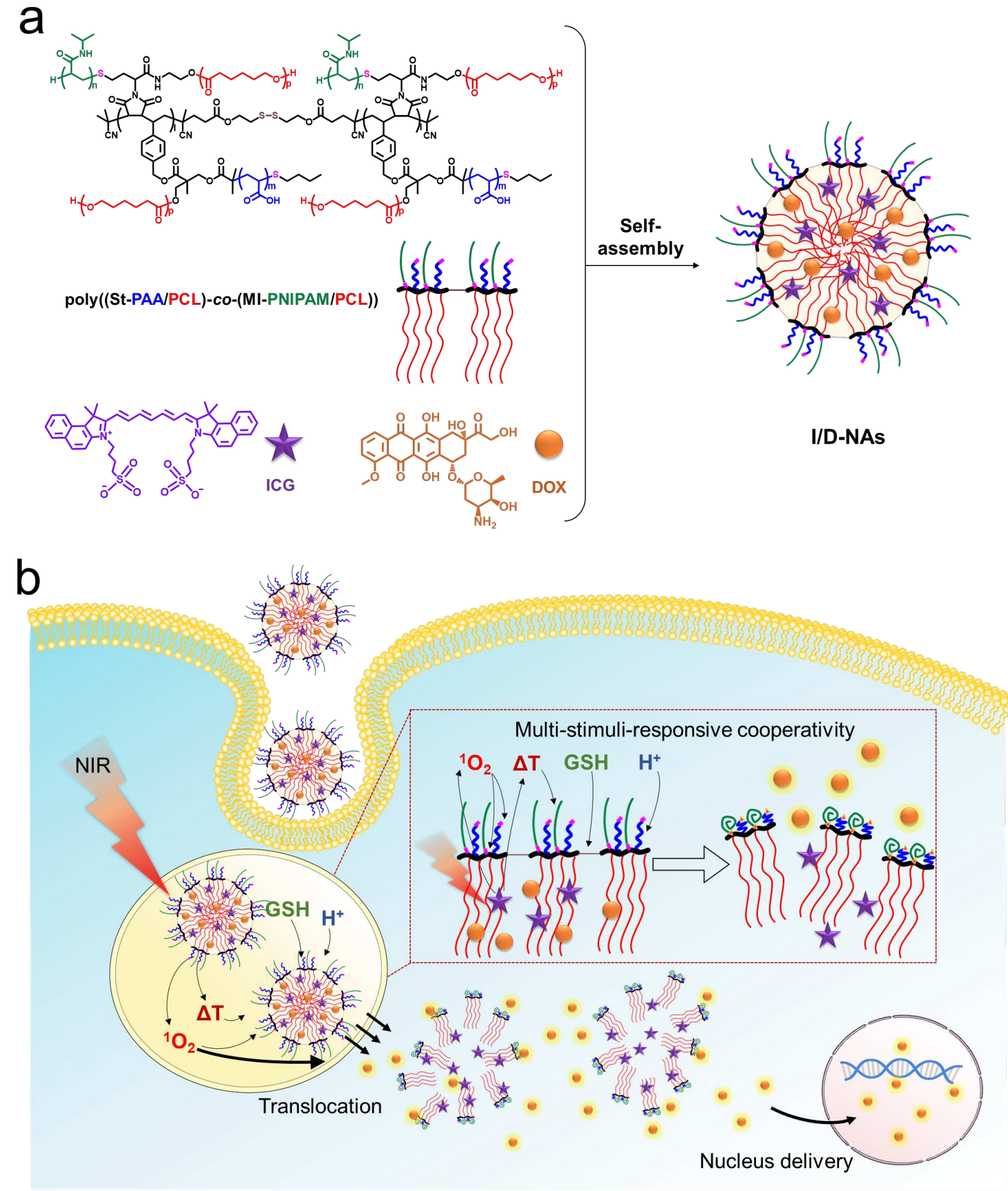

724 Fig. 1. Schematic illustration of nanoassembly for anticancer therapy. a. Construction of H-

725 shaped miktobrush copolymer nanoassemblies with $\mathrm{pH} /$ reduction/temperature/ROS-responsive

726 properties for co-encapsulating representative antitumour compounds such as indocyanine green 
727 (ICG) and doxorubicin (DOX). b. Synergistic photo-chemotherapeutic mechanism against highly

728 aggressive TNBC tumours through multi-stimuli-responsive drug release and ultrafast nucleus

729 delivery.

730 


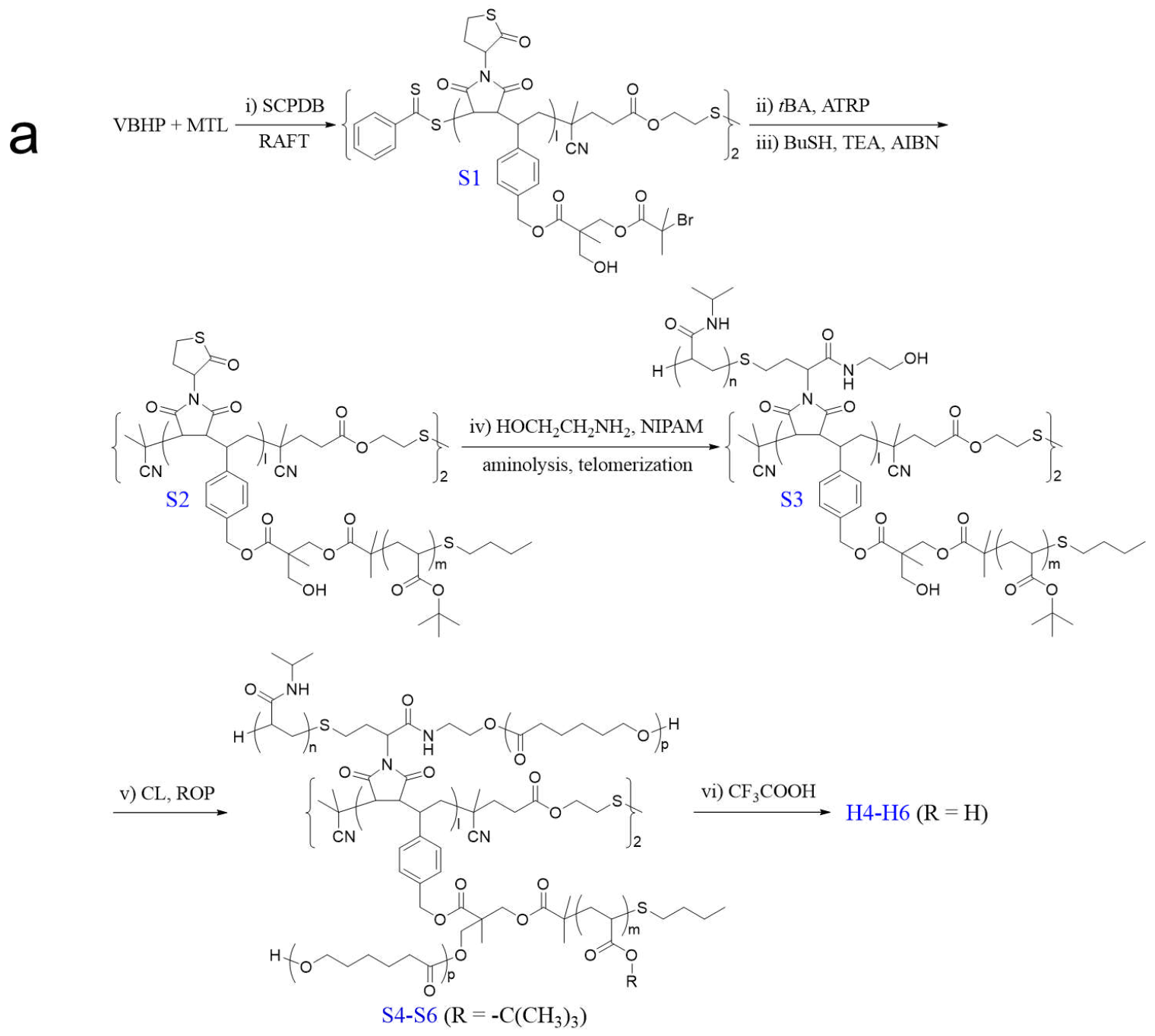

b

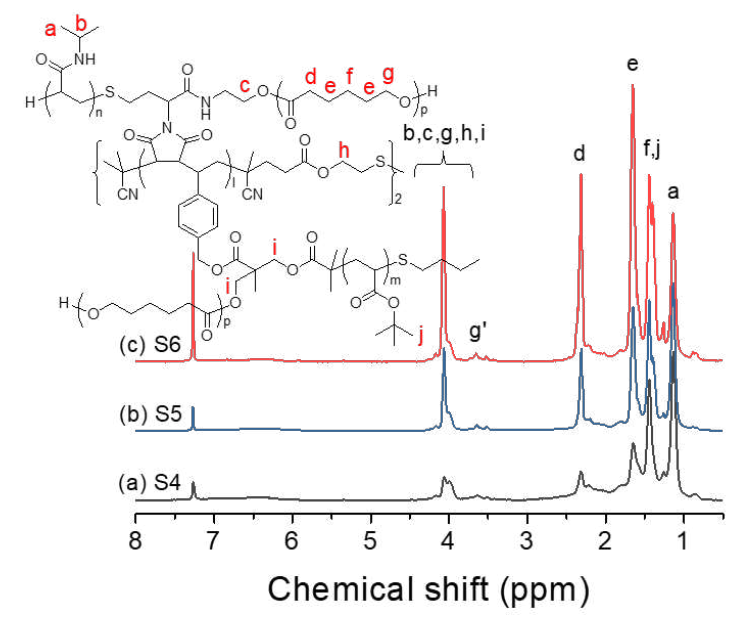

C

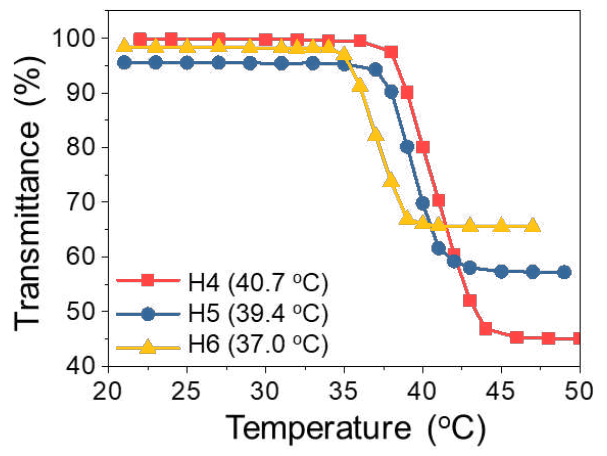

Fig. 2 Synthesis and characterization of brush-like copolymers. a. Synthesis of disulfide-

733 bridged $\mathrm{pH} /$ reduction/temperature/ROS-responsive poly((St-P $t \mathrm{BA} / \mathrm{PCL})-c o-(\mathrm{MI}-\mathrm{PNIPAM} / \mathrm{PCL}))$ 
734 copolymers (H4-H6) by the combination of controlled polymerization, amine-thiol-telomerization 735 and selective hydrolysis, in which $1 \approx 7, \mathrm{~m} \approx 14, \mathrm{n} \approx 25$, and $\mathrm{p} \approx 11$ (S4, H4), 18 (S5, H5) or 73635 (S6, H6). b. ${ }^{1} \mathrm{H}$ NMR spectra of poly((St-PtBA/PCL)-co-(MI-PNIPAM/PCL)) copolymers 737 recorded in $\mathrm{CDCl}_{3}$ at $25{ }^{\circ} \mathrm{C}$, where g' denotes terminal $\mathrm{CH}_{2} \mathrm{OH}$ of PCL grafts. c. Influence of 738 temperature and chain length of PCL on transmittances of copolymer nanoassemblies $\left(c_{\mathrm{p}}=1.0 \mathrm{mg}\right.$ $\left.739 \mathrm{~mL}^{-1}\right)$ formed from disulfide-bridged poly((St-PAA/PCL)-co-(MI-PNIPAM/PCL)) in aqueous 740 solution. 
a

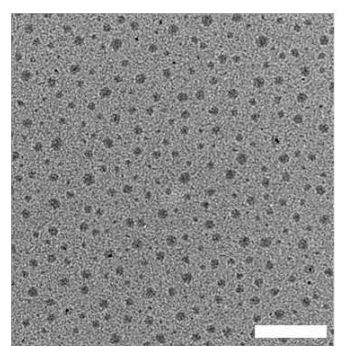

d

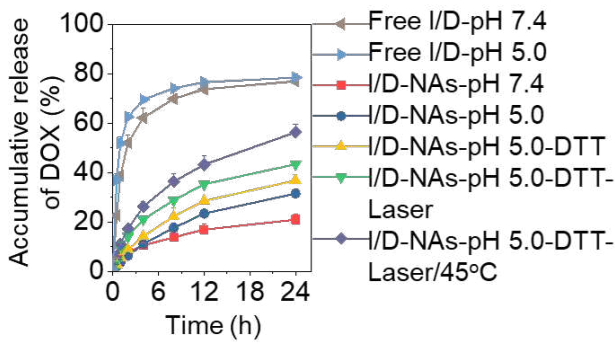

g

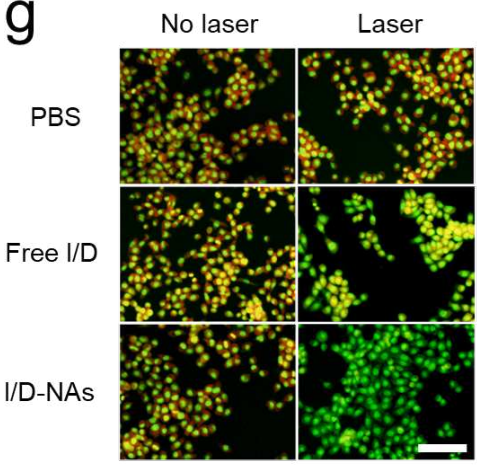

b

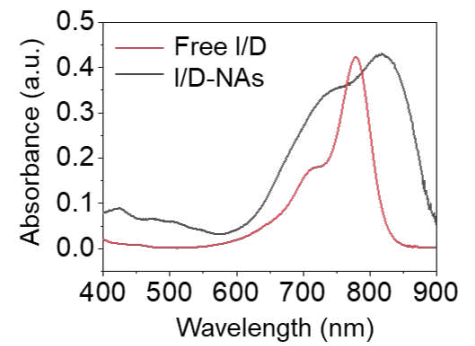

e

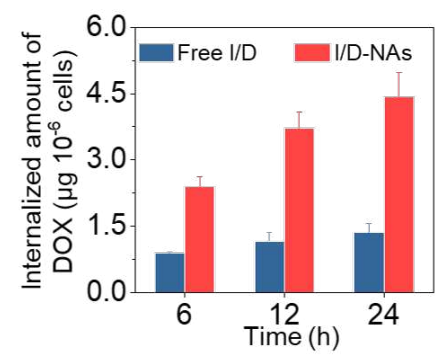

h

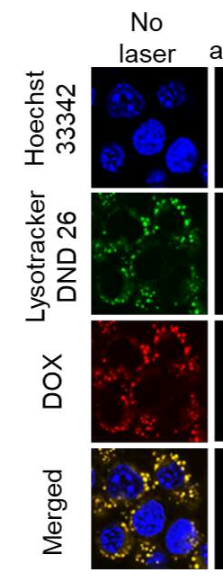

C

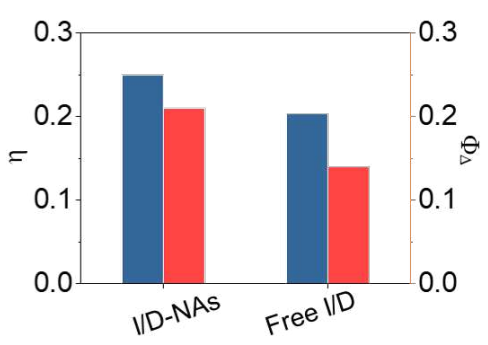

$f$
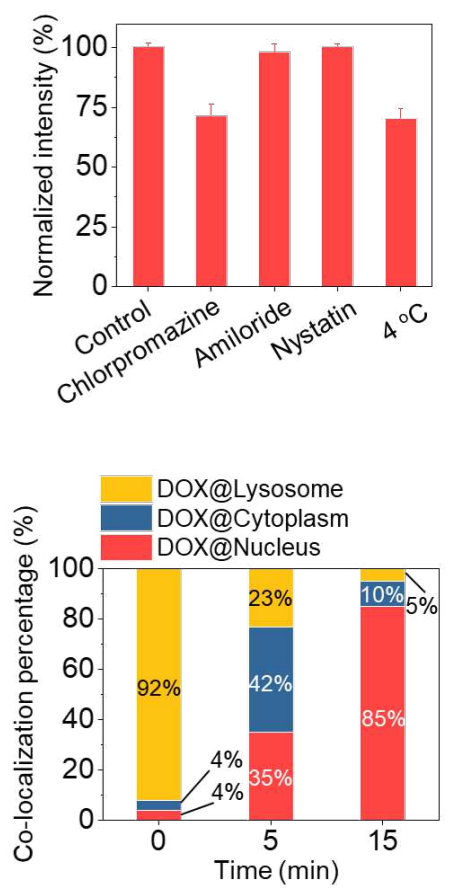

743 Fig. 3. Enhanced lysosome-cytoplasma translocation and rapid nucleus delivery of I/D-NAs

744 by multi-stimuli-responsive cooperativity. a. TEM image of I/D-NAs (scale bar: $500 \mathrm{~nm}$ ). b.

745 UV-vis absorption of Free I/D and I/D-NAs. c. Photothermal conversion efficiency $(\eta)$ and Singlet

746 oxygen quantum yield $\left(\Phi_{\Delta}\right)$ of Free I/D and I/D-NAs under $785 \mathrm{~nm}$ laser irradiation. d.

747 Accumulative releases of DOX from Free I/D and I/D-NAs in various solutions. e. Internalized

748 amounts of DOX in 4T1 cells treated by Free I/D and I/D-NAs at the dose of $10.0 \mu \mathrm{g} \mathrm{mL}^{-1} \mathrm{ICG}$

749 after 6,12 and $24 \mathrm{~h}$ incubation. f. Cellular uptake by $4 \mathrm{~T} 1$ cells treated with various inhibitors. g. 
750 Lysosomal disruption of 4T1 cells treated with Free I/D and I/D-NAs under irradiation or not 751 (scale bar: $20 \mu \mathrm{m}$ ). h. Confocal laser scanning microscopy images (scale bar: $20 \mu \mathrm{m}$ ) of 4T1 cells 752 stained with Lysotracker Green DND-26 and Hoechst 33342 after $2 \mathrm{~h}$ incubation with I/D-NAs at 753 the dose of $10.0 \mu \mathrm{g} \mathrm{ml}^{-1}$ ICG before irradiation, as well as 5 and $15 \mathrm{~min}$ post-irradiation ( $3 \mathrm{~min}$,

$7541.5 \mathrm{~W} \mathrm{~cm}^{-2}$ ), respectively. i. Co-localization percentage of DOX within different organelles as 755 indicated in $\mathbf{h}$.

756 
a

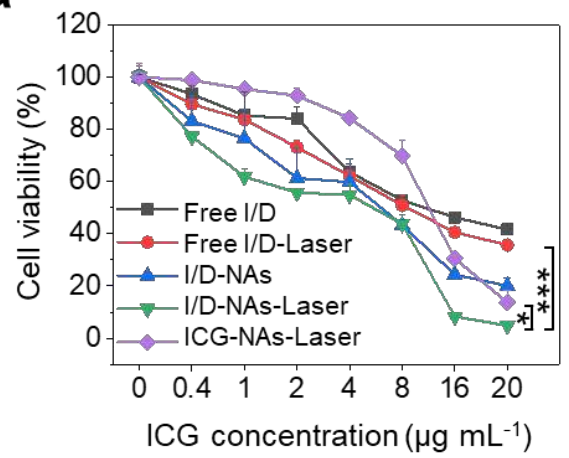

C

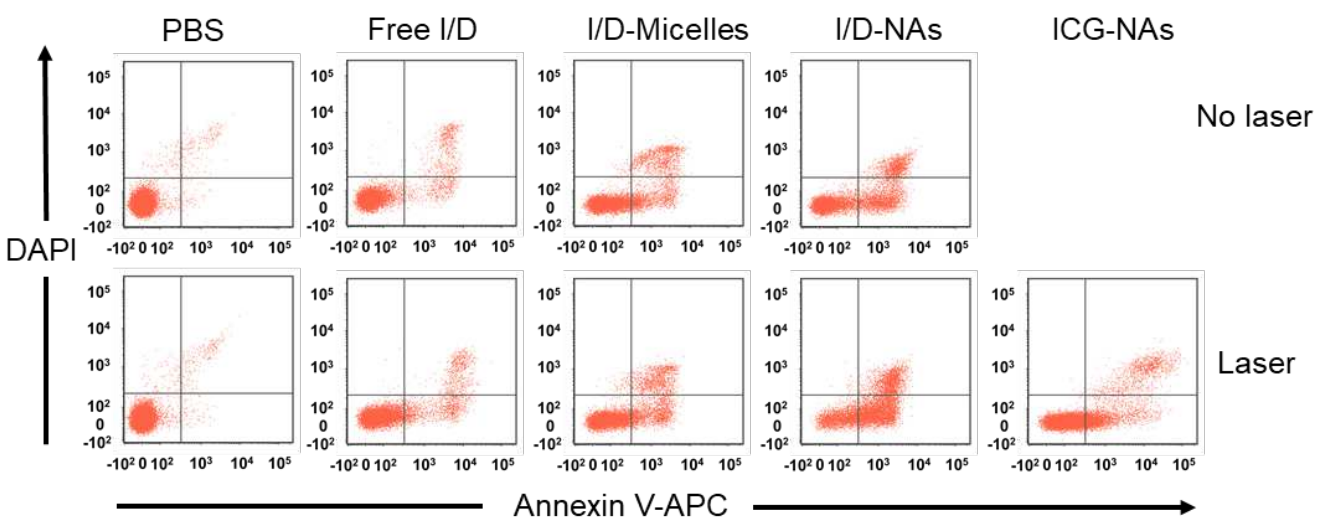

d

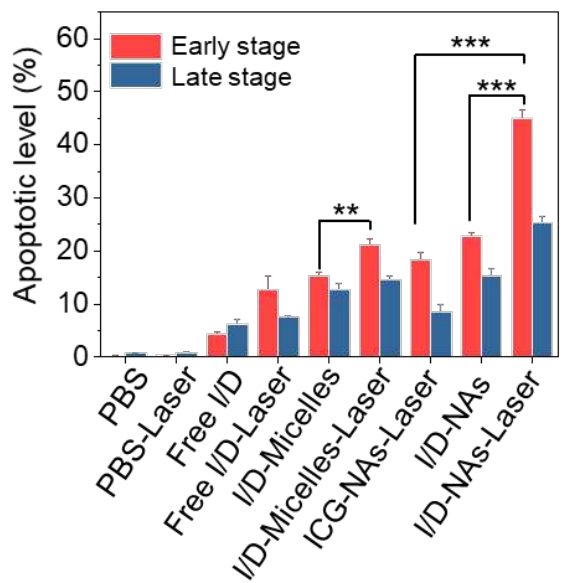

b

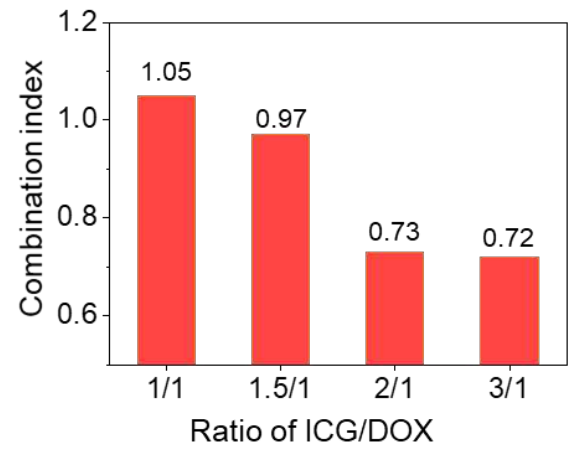

e

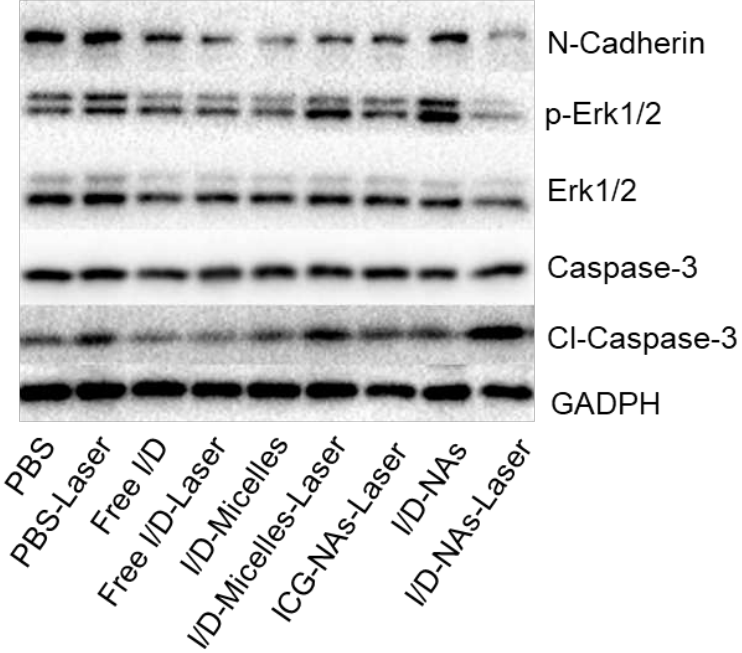

758 Fig. 4. Cooperative cytotoxicity, antitumour and antimetastatic mechanisms of I/D-NAs. a.

759 Cell viability of $4 \mathrm{~T} 1$ cells treated with I/D-NAs and Free I/D under $1.5 \mathrm{~W} \mathrm{~cm}^{-2}$ irradiation (785 nm, $3 \mathrm{~min}$ ) or not. b. Combination index of I/D-NAs at various ratios of ICG:DOX. c. Apoptotic 
761 levels of 4T1 cells stained by Annexin V-APC/DAPI Apoptosis Detection Kit after $24 \mathrm{~h}$ incubation 762 with PBS, Free I/D, I/D-Micelles and I/D-NAs at the dose of $10.0 \mu \mathrm{g} \mathrm{mL} \mathrm{m}^{-1}$ ICG under $3 \mathrm{~min}$ 763 irradiation $\left(785 \mathrm{~nm}, 1.5 \mathrm{~W} \mathrm{~cm}^{-2}\right)$ or not and (d) their apoptotic percentages of early stage and late 764 stage. e. Western blotting of HSP 70, N-cad, p-ERK and Cl-caspase-3 levels at $6 \mathrm{~h}$ post-irradiation 765 in $4 \mathrm{~T} 1$ cells after incubation with PBS, Free I/D, I/D-Micelles and I/D-NAs at the dose of $10.0 \mu \mathrm{g}$ $766 \mathrm{~mL}^{-1} \mathrm{ICG}$ under $3 \mathrm{~min}$ irradiation $\left(785 \mathrm{~nm}, 1.5 \mathrm{~W} \mathrm{~cm}^{-2}\right)$ or not. Statistical analysis was performed 767 using two-sided student's $t$-test, ${ }^{*} p<0.05,{ }^{* *} p<0.01$, and ${ }^{* * *} p<0.001$. 
a

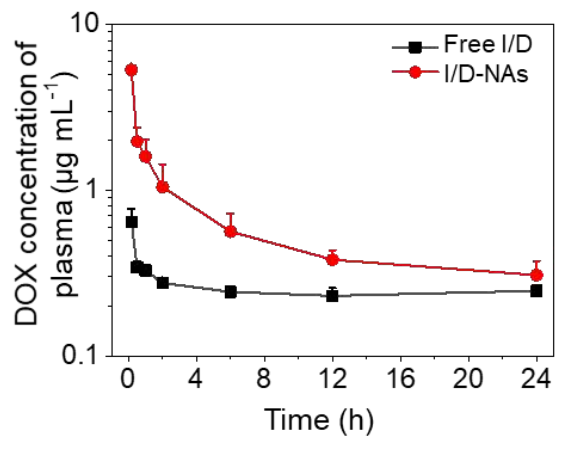

C

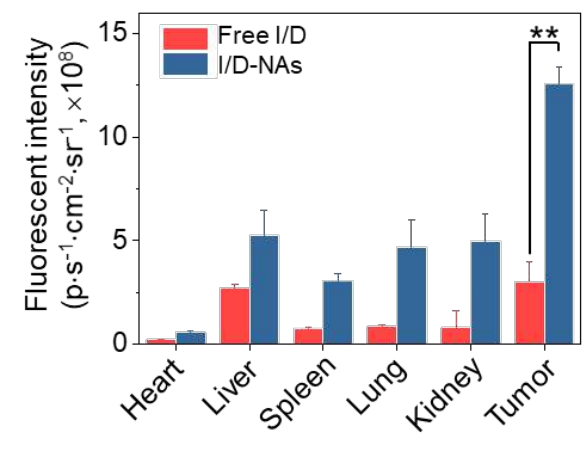

e

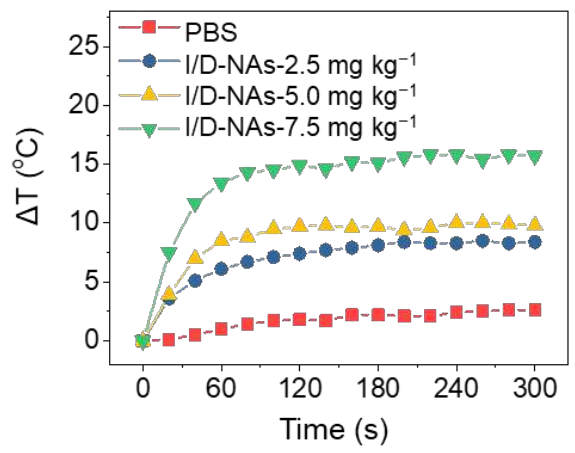

g

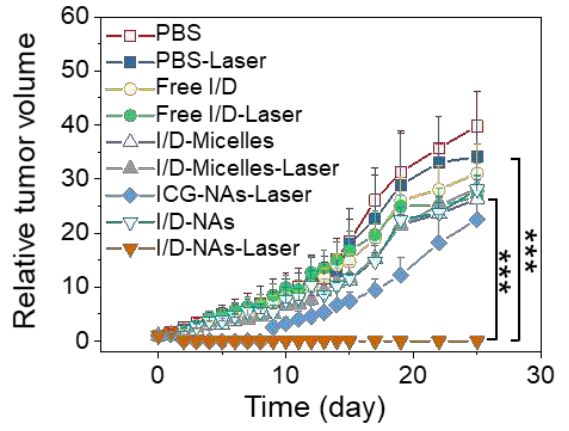

b
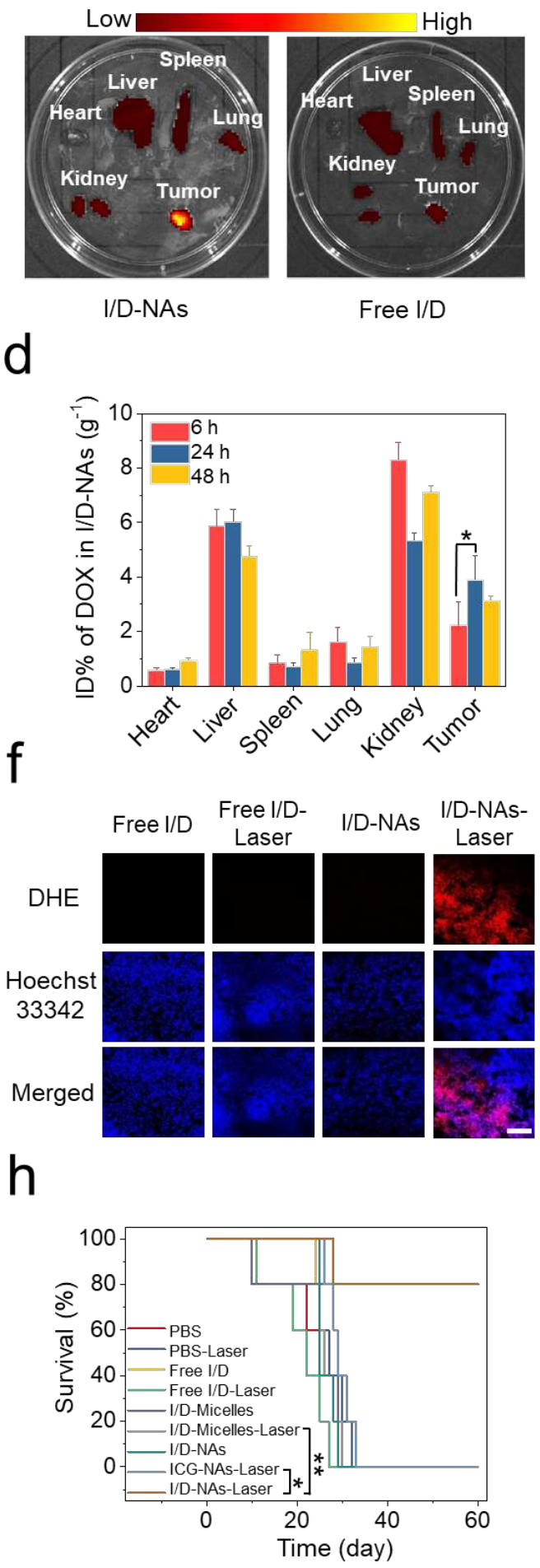

772 Fig. 5. Photochemotherapeutic synergy of I/D-NAs against subcutaneous TNBC tumour

773 models caused by multi-stimuli responsiveness. a. DOX concentrations in plasma from Free I/D 
774 and I/D-NAs at the dose of $7.5 \mathrm{mg} \mathrm{kg}^{-1}$ ICG. b. Ex vivo NIRF images of heart, liver, spleen, lung, 775 kidney, and tumour extracted from the mice bearing $4 \mathrm{~T} 1$ cells treated with Free I/D and I/D-NAs 776 at the dose of $7.5 \mathrm{mg} \mathrm{kg}^{-1}$ ICG and (c) their NIRF intensities. d. Biodistribution of DOX in heart,

777 liver, spleen, lung, kidney, and tumour of the mice bearing 4T1 subcutaneous tumours treated with 778 I/D-NAs at different time post-injection at the dose of $7.5 \mathrm{mg} \mathrm{kg}^{-1}$ ICG. e. Temperature elevations 779 of the mice bearing $4 \mathrm{~T} 1$ cells treated with I/D-NAs at different doses of ICG under $785 \mathrm{~nm}$ 780 irradiation at $1.5 \mathrm{~W} \mathrm{~cm}^{-2}$. f. DHE staining of tumour sections treated with Free I/D and I/D-NAs 781 at the dose of $7.5 \mathrm{mg} \mathrm{kg}^{-1}$ ICG under 3 min irradiation or not (scale bar: $100 \mu \mathrm{m}$ ). g. Tumour 782 growth profiles of the mice bearing 4T1 subcutaneous tumours with various treatments at the dose 783 of $7.5 \mathrm{mg} \mathrm{kg}^{-1} \mathrm{ICG}$, and (h) their Kaplan-Meier survival plots of the mice. The statistical analysis 784 was determined by two-sided student's $t$-test, ${ }^{* *} p<0.01$ and ${ }^{* * *} p<0.001$. The statistical analysis 785 was carried out using two-sided log-rank (Mantel-Cox) test for $\mathbf{h},{ }^{*} p<0.05$ and ${ }^{* *} p<0.01$. 786 


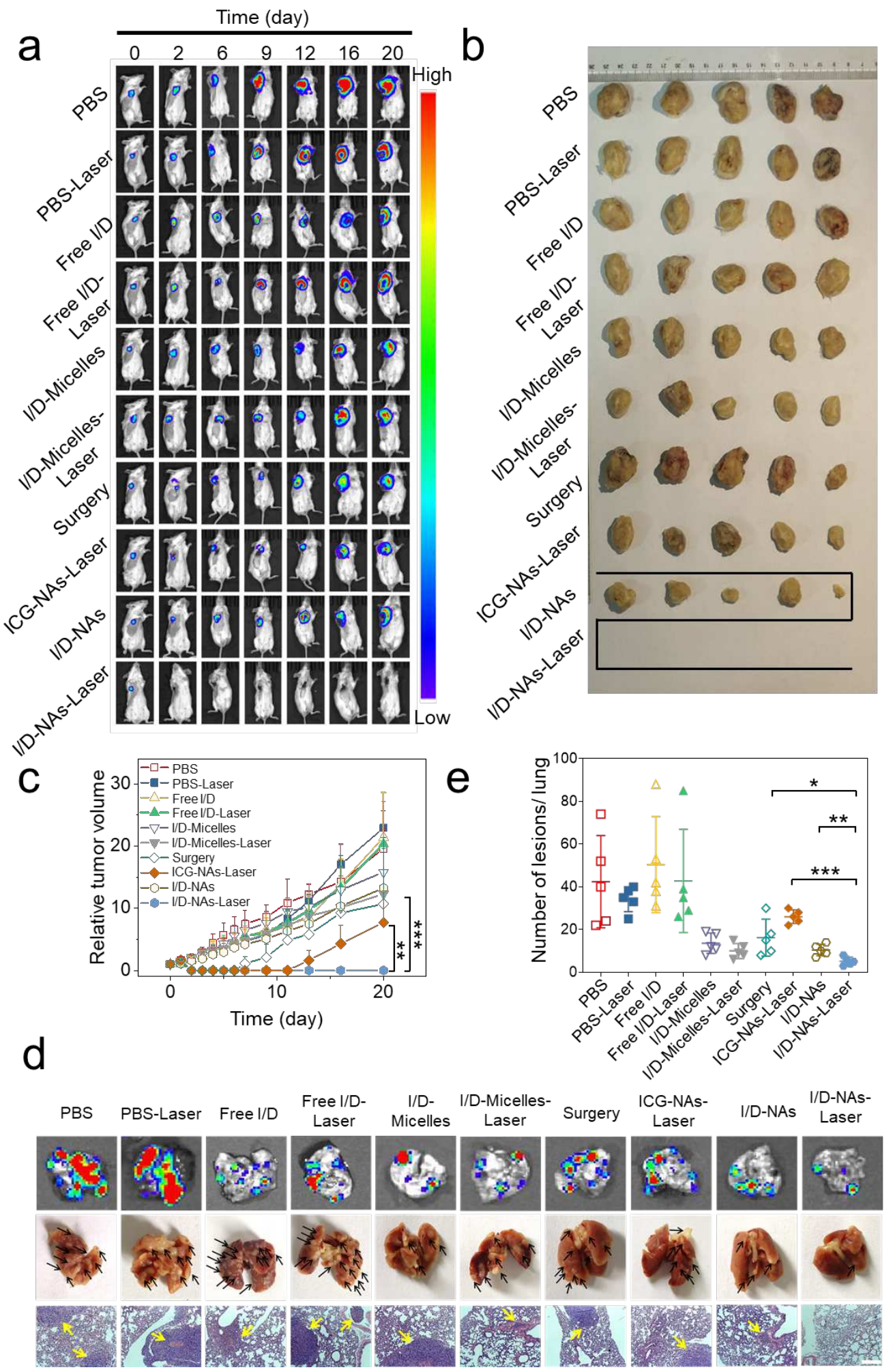

Fig. 6. Photochemotherapeutic synergy of I/D-NAs against orthotopic and metastatic TNBC

789 tumour models. a. In vivo bioluminescence images to track the tumour growth of the mice bearing 
790 orthotopic $4 \mathrm{~T} 1$ breast tumours after various treatments. b. The photographs of tumours extracted 791 from the mice at 20 days post-injection as indicated in a. c. Tumour growth profiles of mice with 792 various treatments as indicated in a. d. Exo vivo bioluminescence images, optical photographs and 793 H\&E staining images (scale bar: $200 \mu \mathrm{m}$ ) of the lungs from the mice at 20 days post-injection as 794 indicated in a. e. Counts of metastatic nodules in the lungs of the mice at 20 days post-injection as 795 indicated in a. Statistical analysis was determined by two-sided student's $t$-test, ${ }^{*} p<0.05,{ }^{* *} p<$ 7960.01 and $^{* * *} p<0.001$.

797 
800 H-shaped miktobrush copolymer nanoassembly facilitates 801 ultrafast nucleus delivery for multi-stimuli-cooperative 802 tumour suppression

803 Miya Zhang ${ }^{1,2, \#}$, Wenxue Dai ${ }^{3, \#}$, Tao Yang ${ }^{1,2}$, Ting $\mathrm{Li}^{1,2}$, Tao $\mathrm{Xu}^{1,2}$, Yibin Deng ${ }^{1,2}$, Hong Yang ${ }^{1}$, 804 Hengte $\mathrm{Ke}^{1,2, *}$, Youliang Zhao ${ }^{3, *}$, and Huabing Chen ${ }^{1,2, *}$

$805{ }^{1}$ State Key Laboratory of Radiation Medicine and Protection, Soochow University, Suzhou 215123, 806 China

807 2Jiangsu Key Laboratory of Neuropsychiatric Diseases, and College of Pharmaceutical Sciences, 808 Soochow University, Suzhou 215123, China

$809{ }^{3}$ Suzhou Key Laboratory of Macromolecular Design and Precision Synthesis, Jiangsu Key 810 Laboratory of Advanced Functional Polymer Design and Application, State and Local Joint 811 Engineering Laboratory for Novel Functional Polymeric Materials, College of Chemistry, 812 Chemical Engineering and Materials Science, Soochow University, Suzhou 215123, China

815 Keywords: polymer nanoassembly, multi-stimuli responsiveness, nucleus delivery, triple-

816 negative breast cancer, photo-chemotherapy

$817{ }^{\#}$ These authors contributed equally to this work.

$818{ }^{*}$ Correspondence and requests for materials should be addressed to H.C. (chenhb@suda.edu.cn), 819 Y.Z. (ylzhao@suda.edu.cn), H.K. (htke@suda.edu.cn). 
1. Supplementary Figures and Tables

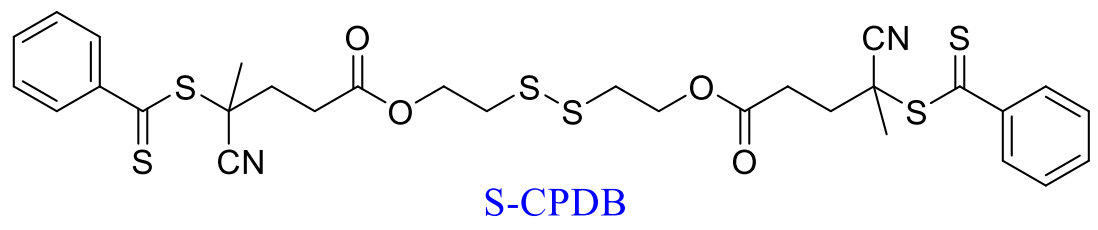

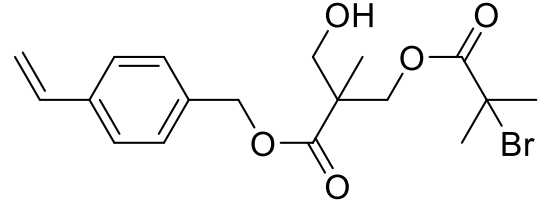

VBHP<smiles>O=C1SCCC1N1C(=O)C=CC1=O</smiles>

MTL

823 Supplementary Fig. 1. Chemical structures of functional reagents S-CPDB, VBHP and MTL.

824 


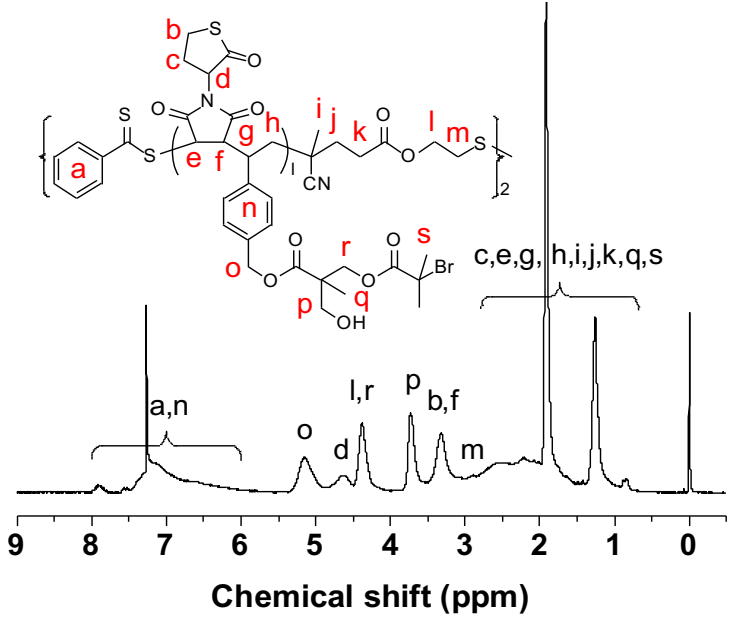

826 Supplementary Fig. 2. ${ }^{1} \mathrm{H}$ NMR spectrum of P(MTL-co-VBP) (S1) recorded in $\mathrm{CDCl}_{3}$ at $25^{\circ} \mathrm{C}$. 827 


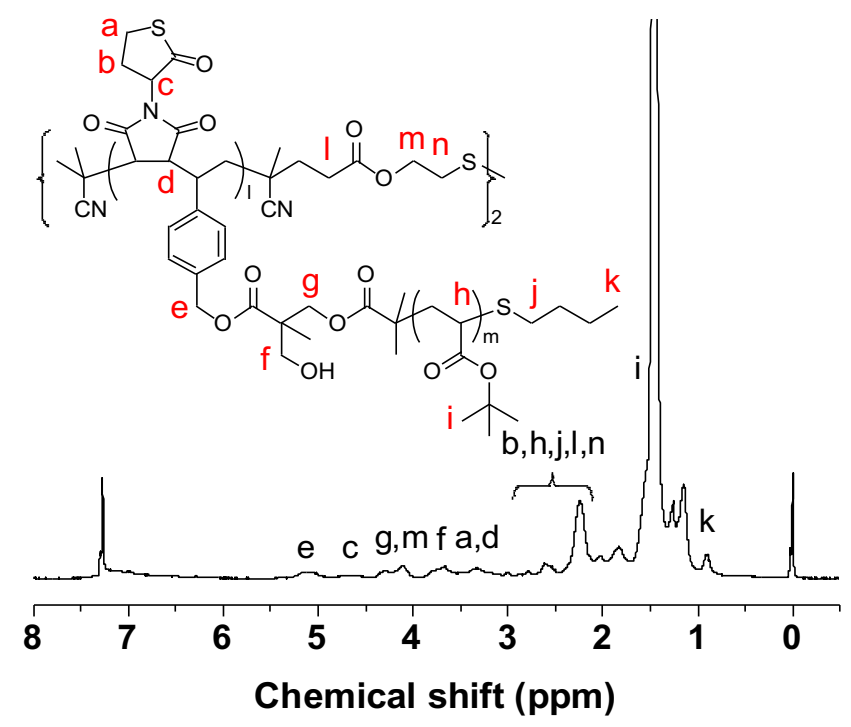

828

829 Supplementary Fig. 3. ${ }^{1} \mathrm{H}$ NMR spectrum of $\mathrm{P}((\mathrm{St}-\mathrm{P} t \mathrm{BA})-c o-\mathrm{MTL})(\mathrm{S} 2)$ recorded in $\mathrm{CDCl}_{3}$ at $83025^{\circ} \mathrm{C}$.

831 


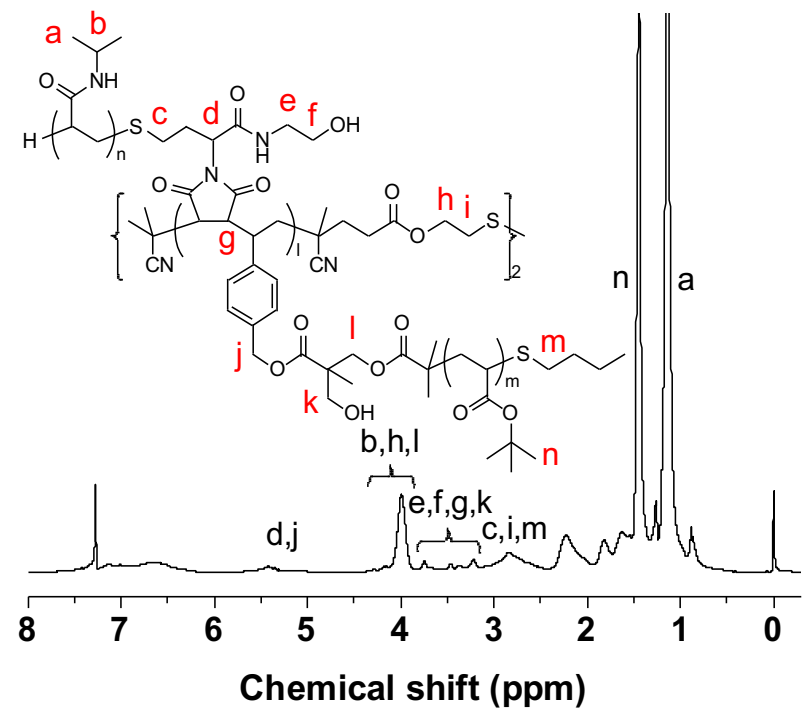

832

833 Supplementary Fig. 4. ${ }^{1} \mathrm{H}$ NMR spectrum of poly((St-P $t$ BA)-co-(MI-PNIPAM)) (S3) recorded in $834 \mathrm{CDCl}_{3}$ at $25^{\circ} \mathrm{C}$.

835 


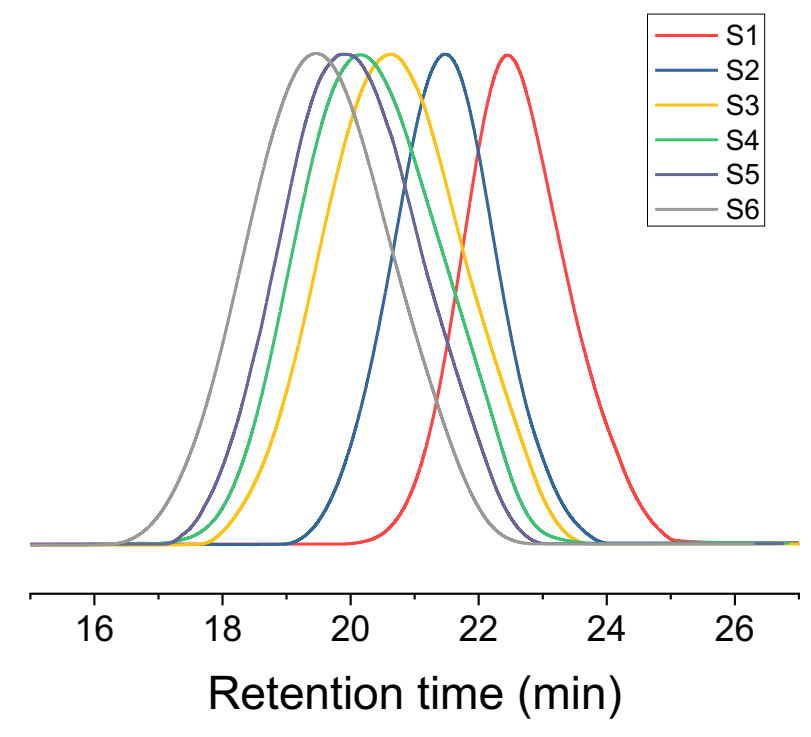

836

837 Supplementary Fig. 5. GPC traces of poly(VBHP-co-MTL) (S1), poly((St-PtBA)-co-MTL) (S2), 838 poly((St-PtBA)-co-(MI-PNIPAM)) (S3), and poly((St-PtBA/PCL)-co-(MI-PNIPAM/PCL)) (S4839 S6) copolymers.

840 


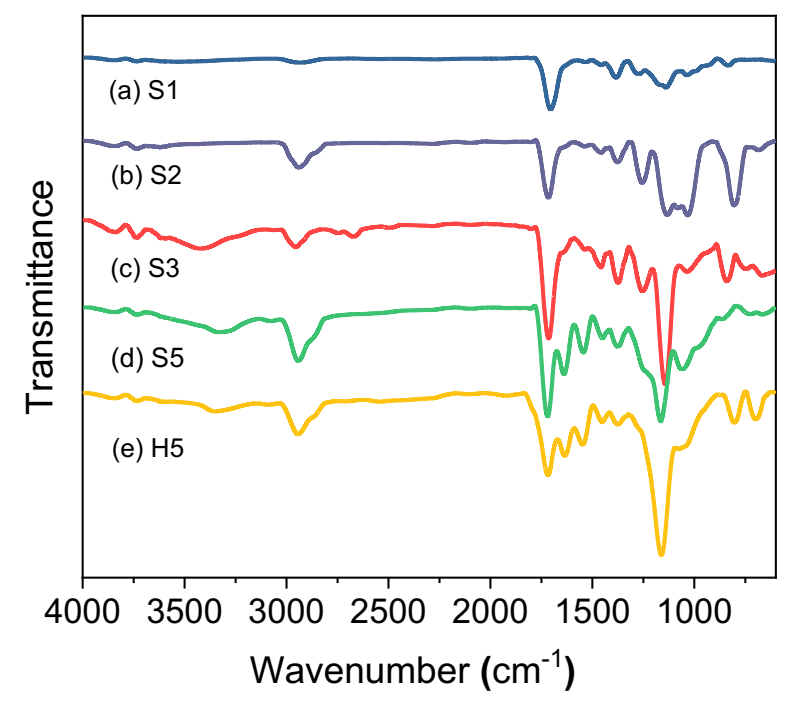

842 Supplementary Fig. 6. FT-IR spectra of poly(VBHP-co-MTL) (S1, a), poly((St-PtBA)-co-MTL)

843 (S2, b), poly((St-PtBA)-co-(MI-PNIPAM)) (S3, c), poly((St-PtBA/PCL)-co-(MI-PNIPAM/PCL))

844 (S5, d), and poly((St-PAA/PCL)-co-(MI-PNIPAM/PCL)) (H5, e).

845 


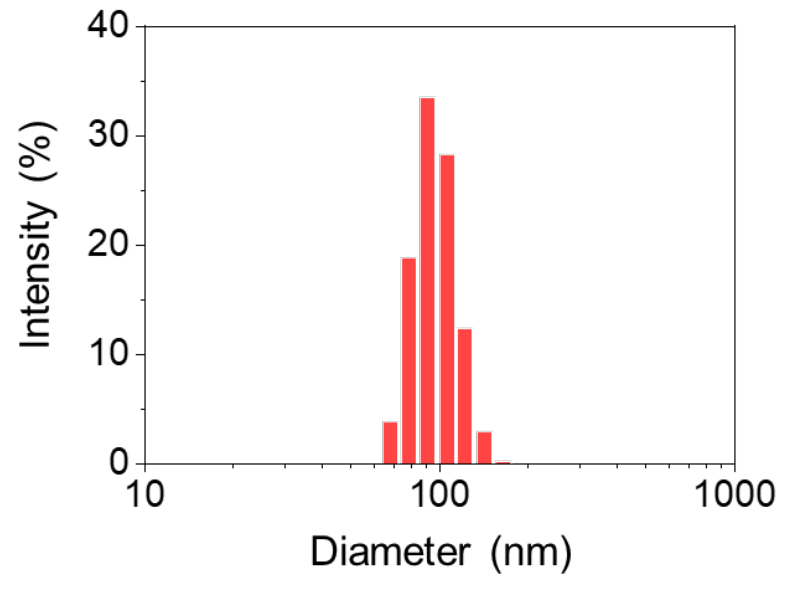

846

847 Supplementary Fig. 7. Size distribution of I/D-NAs using DLS.

848 


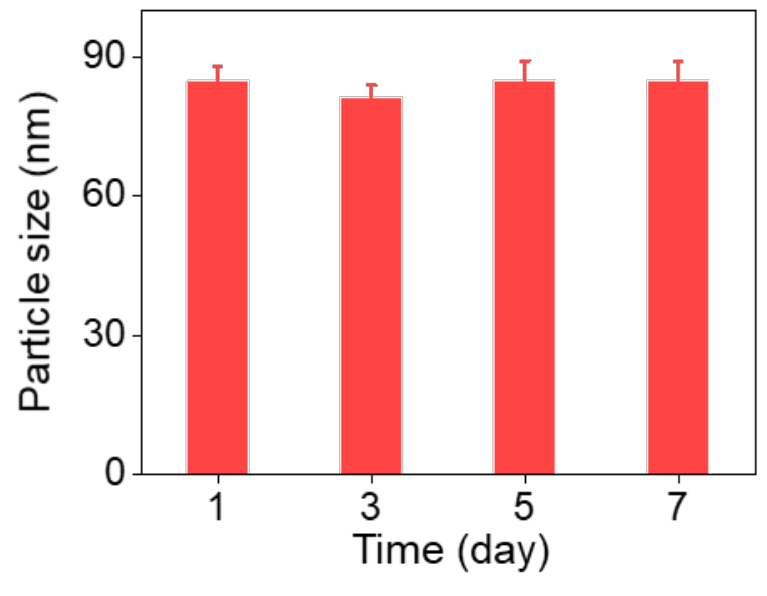

849

850 Supplementary Fig. 8. Particle size of I/D-NAs at 1, 3, 5 and 7 days using DLS. 


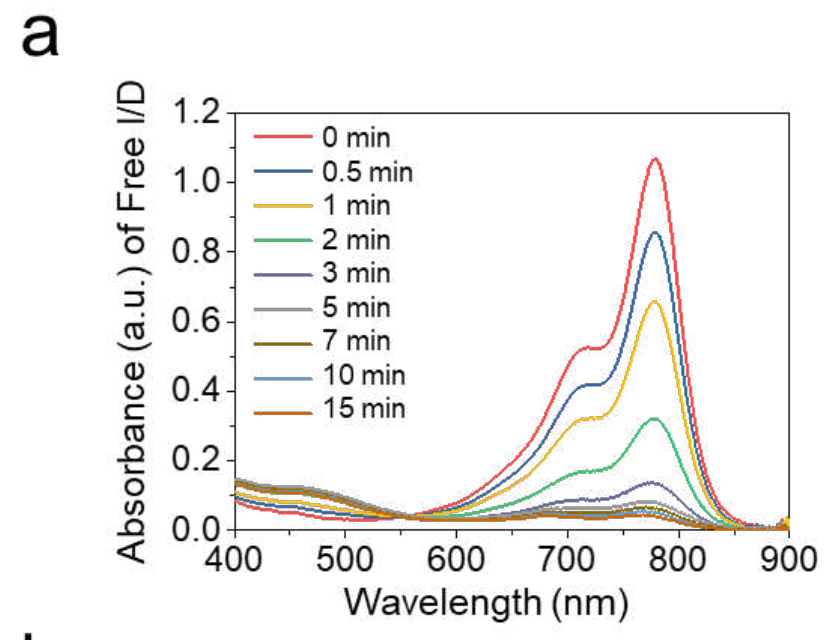

b
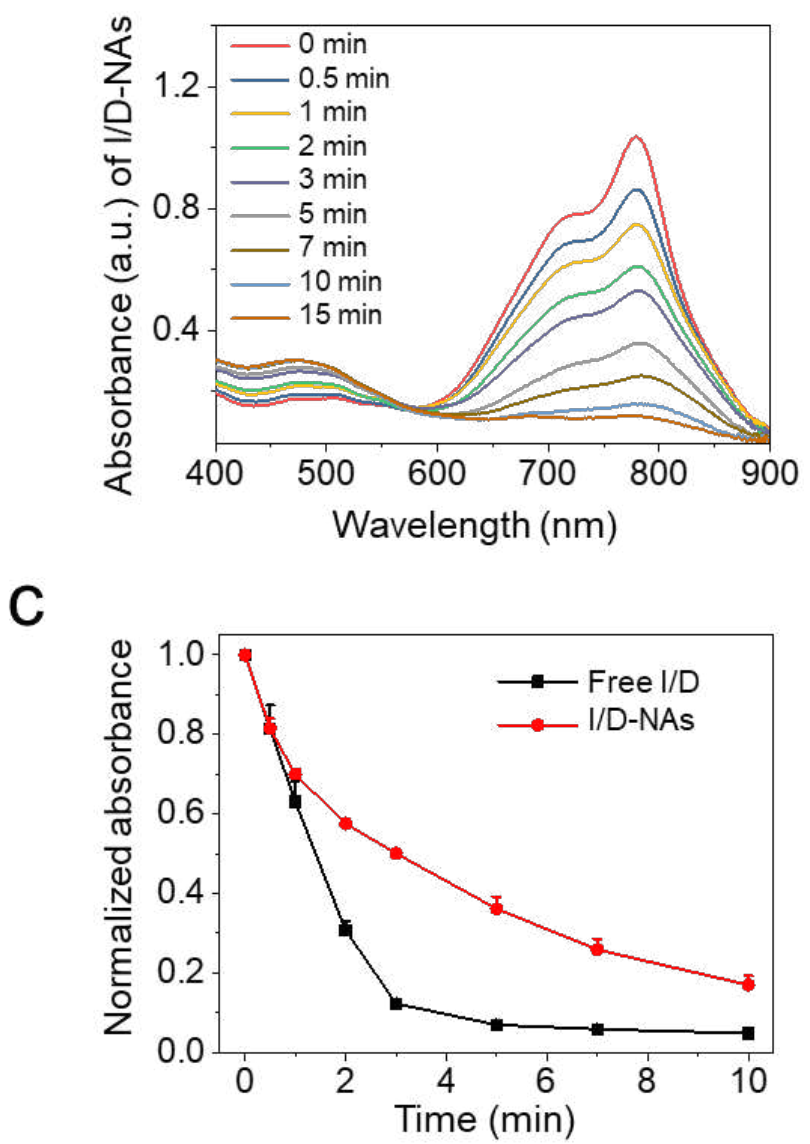

852

853 Supplementary Fig. 9. The UV absorbance of Free I/D (a) and I/D-NAs (b) at different time after 854 light irradiation $\left(785 \mathrm{~nm}, 1.5 \mathrm{~W} \mathrm{~cm}^{-2}\right)$. c. Normalized absorbance of Free I/D and I/D-NAs at 10.0 $855 \mu \mathrm{g} \mathrm{mL}^{-1} \mathrm{ICG}$ at different time under irradiation $\left(785 \mathrm{~nm}, 1.5 \mathrm{~W} \mathrm{~cm}^{-2}\right)$. 


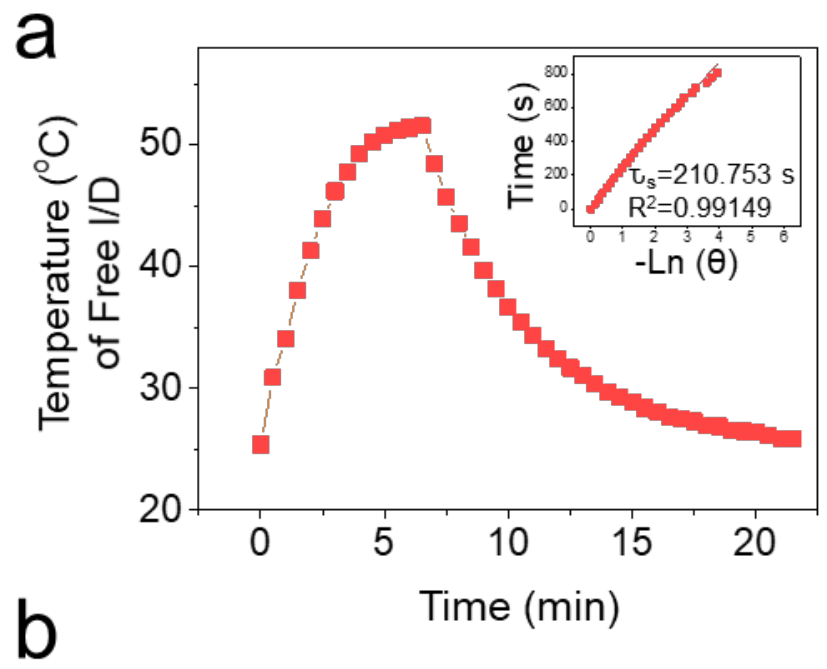

857

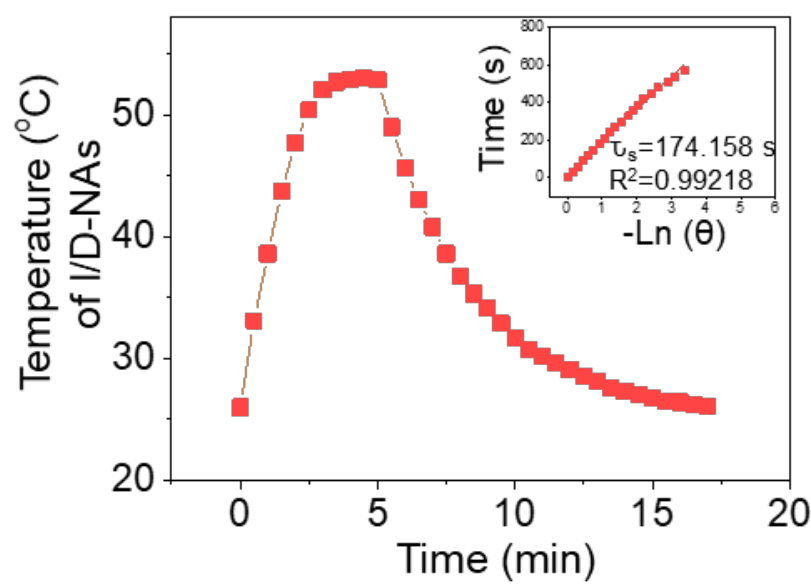

858 Supplementary Fig. 10. Temperature elevation of Free I/D (a) and I/D-NAs (b) with $10.0 \mu \mathrm{g} \mathrm{mL}^{-}$

$859{ }^{1}$ ICG under $785 \mathrm{~nm}$ irradiation $\left(1.5 \mathrm{~W} \mathrm{~cm}^{-2}\right)$, and subsequent natural cooling when removing the 860 irradiation.

861 

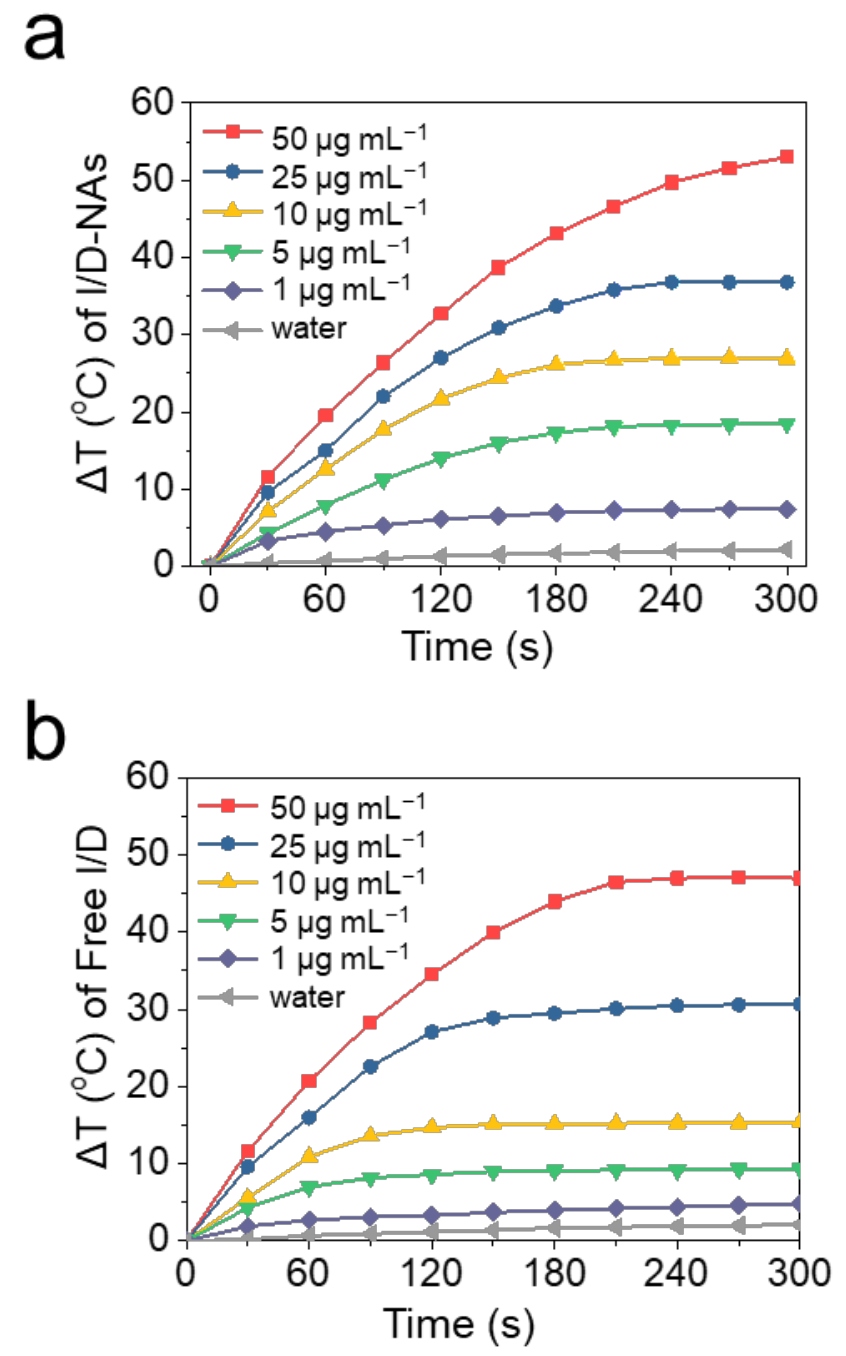

862

863 Supplementary Fig. 11. Temperature elevations of I/D-NAs (a) and Free I/D (b) at various 864 concentrations of ICG within 5 min irradiation $\left(785 \mathrm{~nm}, 1.5 \mathrm{~W} \mathrm{~cm}^{-2}\right)$.

865 


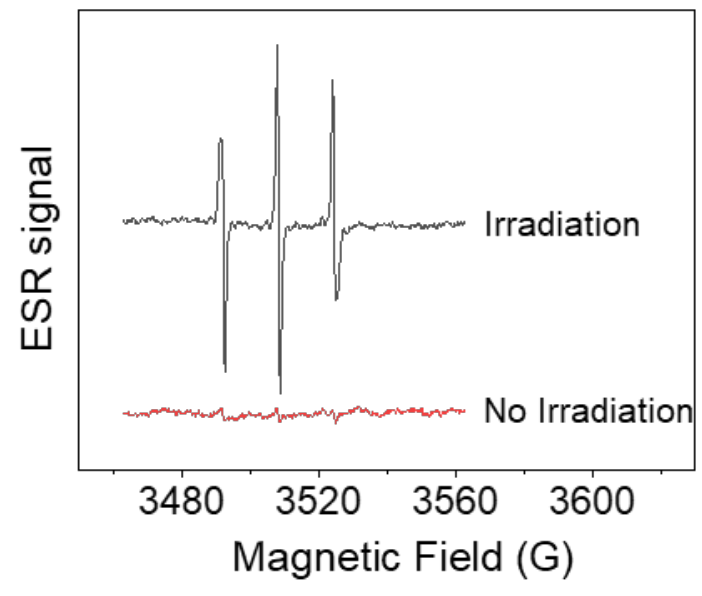

867 Supplementary Fig. 12. ESR spectra of I/D-NAs using TEMP as spin-trapping agent under 785

$868 \mathrm{~nm}$ irradiation $\left(1.5 \mathrm{~W} \mathrm{~cm}^{-2}, 5 \mathrm{~min}\right)$.

869 


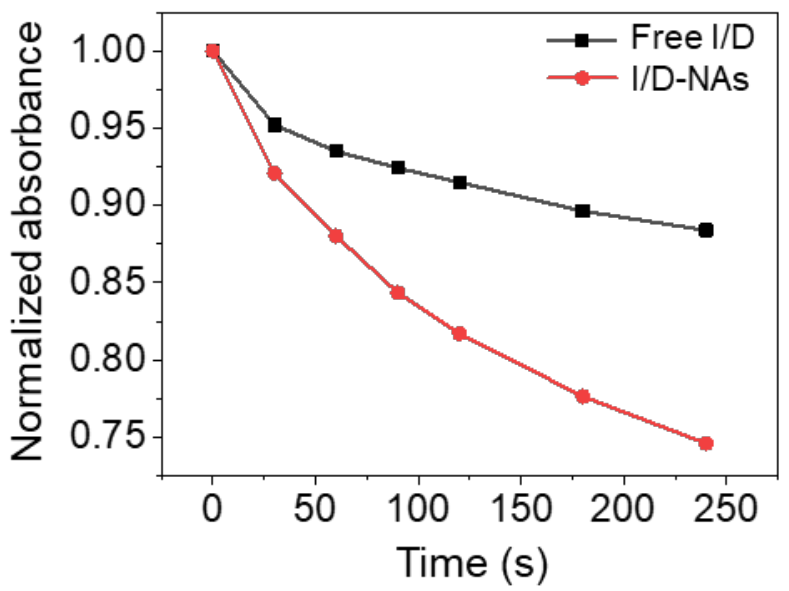

871 Supplementary Fig. 13. Normalized absorbances of Free I/D and I/D-NAs at various time using 872 DPBF as a probe under $785 \mathrm{~nm}$ irradiation at $1.5 \mathrm{~W} \mathrm{~cm}^{-2}$.

873 


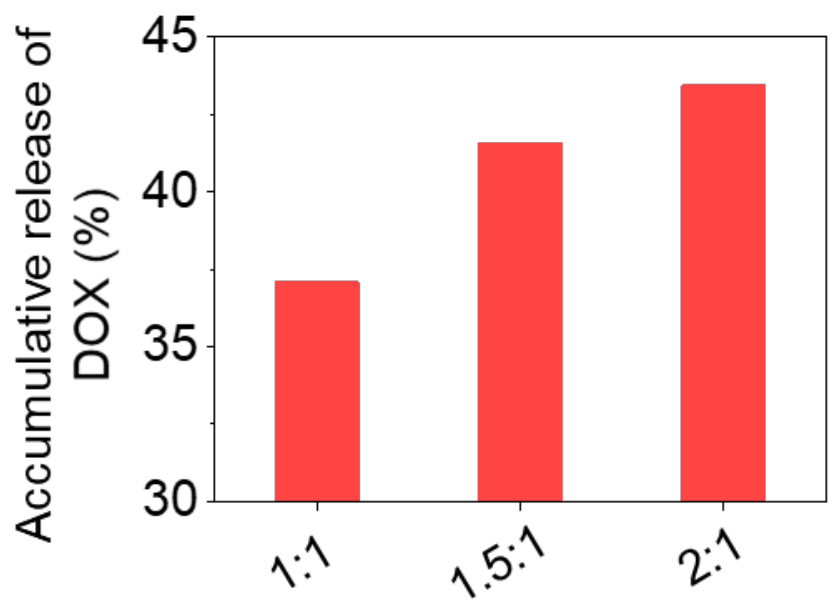

874

875 Supplementary Fig. 14. Accumulative releases of DOX from I/D-NAs at various ICG/DOX ratios 876 at the concentration of $80.0 \mu \mathrm{g} \mathrm{mL}^{-1} \mathrm{DOX}$ in the presence of $10.0 \mathrm{mM}$ DTT under $3 \mathrm{~min}$ light 877 irradiation during $24 \mathrm{~h}$.

878 

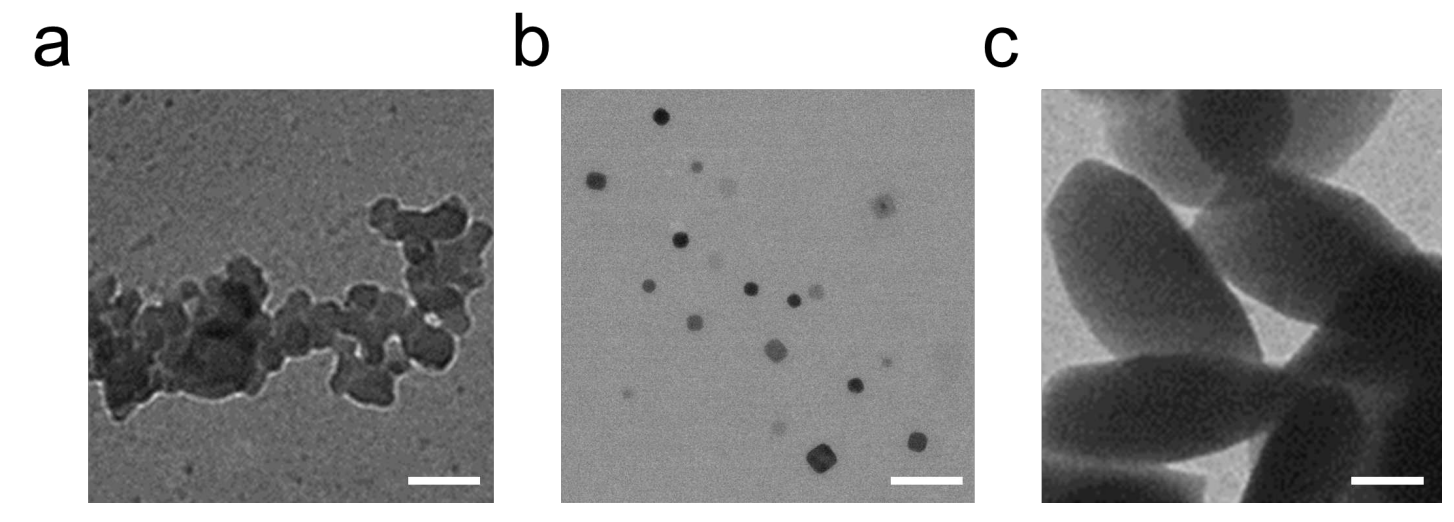

880 Supplementary Fig. 15. TEM images of I/D-NAs in various stimulative environments including 881 acidic solution of $\mathrm{pH} 5.0$ (a), reduction solution with DTT after $12 \mathrm{~h}$ reaction time (b), and light 882 irradiation for $5 \mathrm{~min}\left(785 \mathrm{~nm}, 1.5 \mathrm{~W} \mathrm{~cm}^{-2}\right)(\mathbf{c})$. (scale bar: $\left.100 \mathrm{~nm}\right)$. 


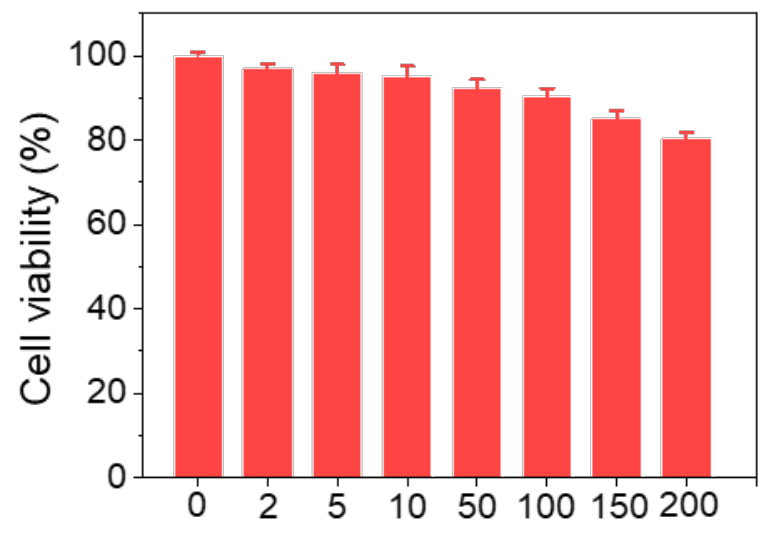

Empty nanoassemblies concentration

$\left(\mu \mathrm{g} \mathrm{mL} \mathrm{L}^{-1}\right)$

884

885 Supplementary Fig. 16. Cell viability of 4T1 cells treated with drug-free NAs.

886 


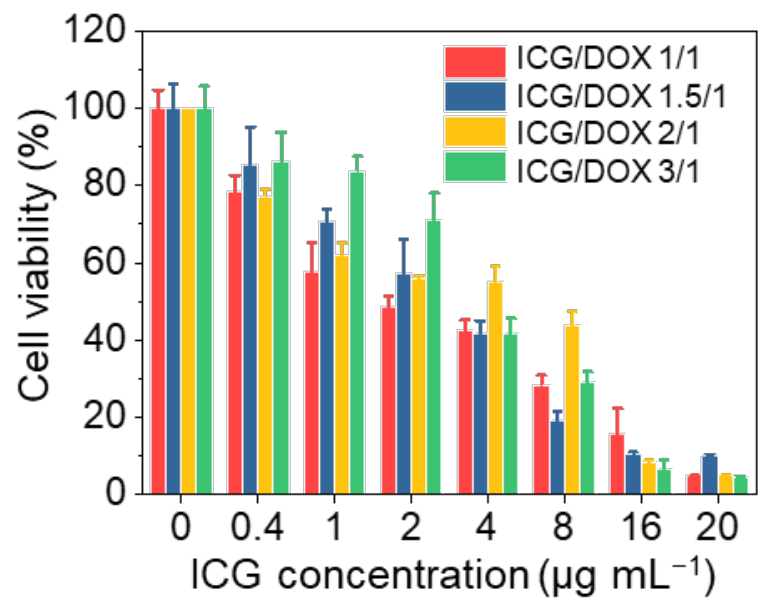

888 Supplementary Fig. 17. Cell viability of $4 \mathrm{~T} 1$ cells treated I/D-NAs with the ICG/DOX ratios of $8893 / 1,2 / 1,1.5 / 1$, and $1 / 1$ at the different doses of ICG under $1.5 \mathrm{~W} \mathrm{~cm}^{-2}$ irradiation for $3 \mathrm{~min}$ or not. 890 


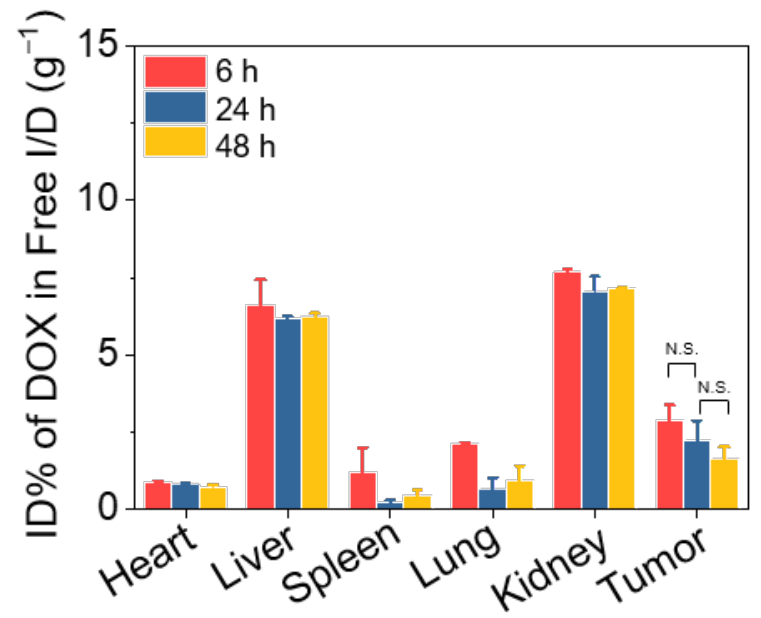

891

892 Supplementary Fig. 18. Ex vivo distribution of DOX in heart, liver, spleen, lung, kidney, and 893 tumour of the mice bearing 4T1 subcutaneous tumours treated with Free I/D at different time post894 injection at the dose of $7.5 \mathrm{mg} \mathrm{kg}^{-1}$ ICG. Statistical analysis was performed using two-sided 895 student's $t$-test, $p>0.05$.

896 

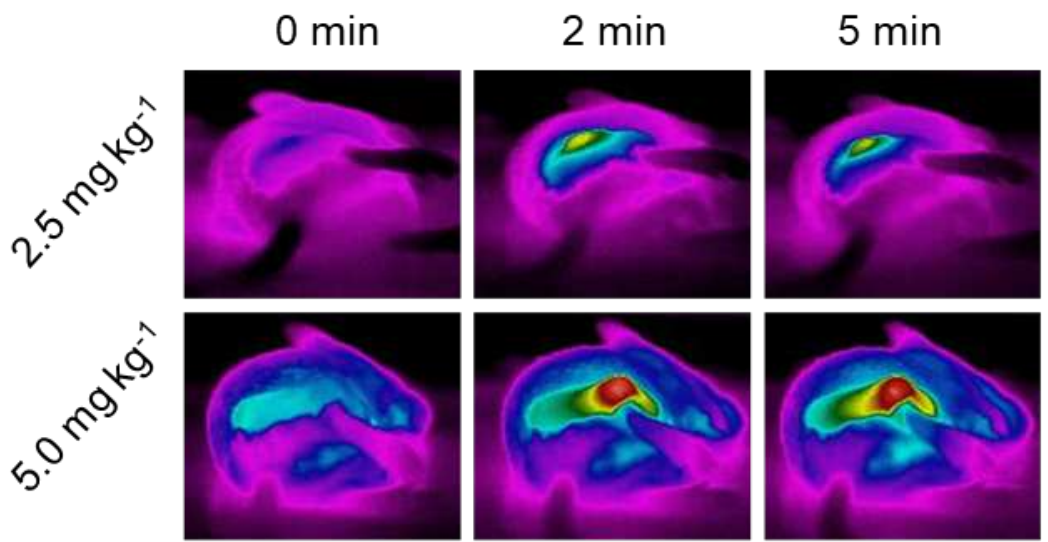

Max

897
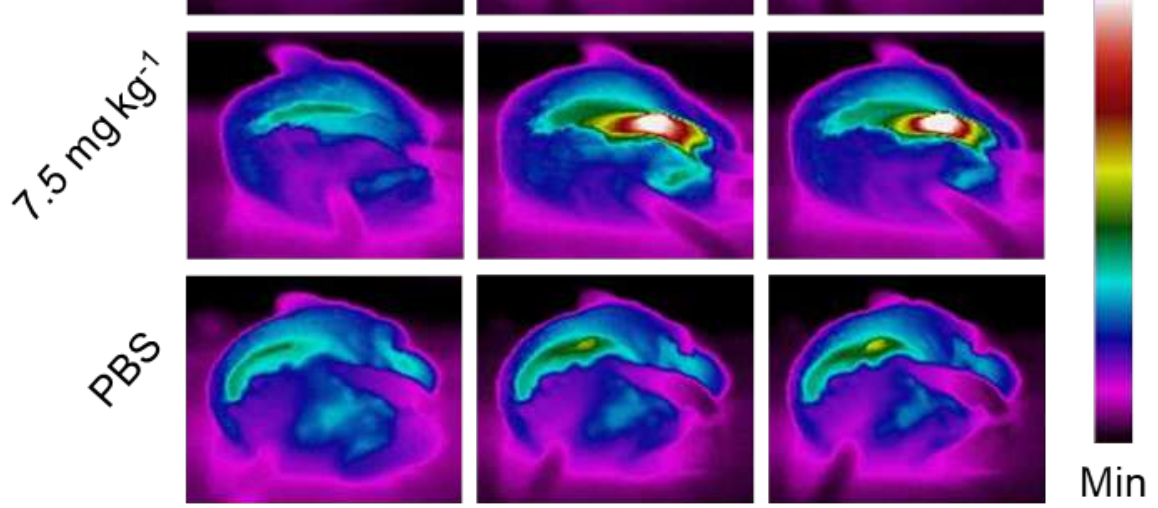

898 Supplementary Fig. 19. Infrared thermography of the mice bearing 4T1 cells treated with I/D-

899 NAs at different doses of ICG.

900 


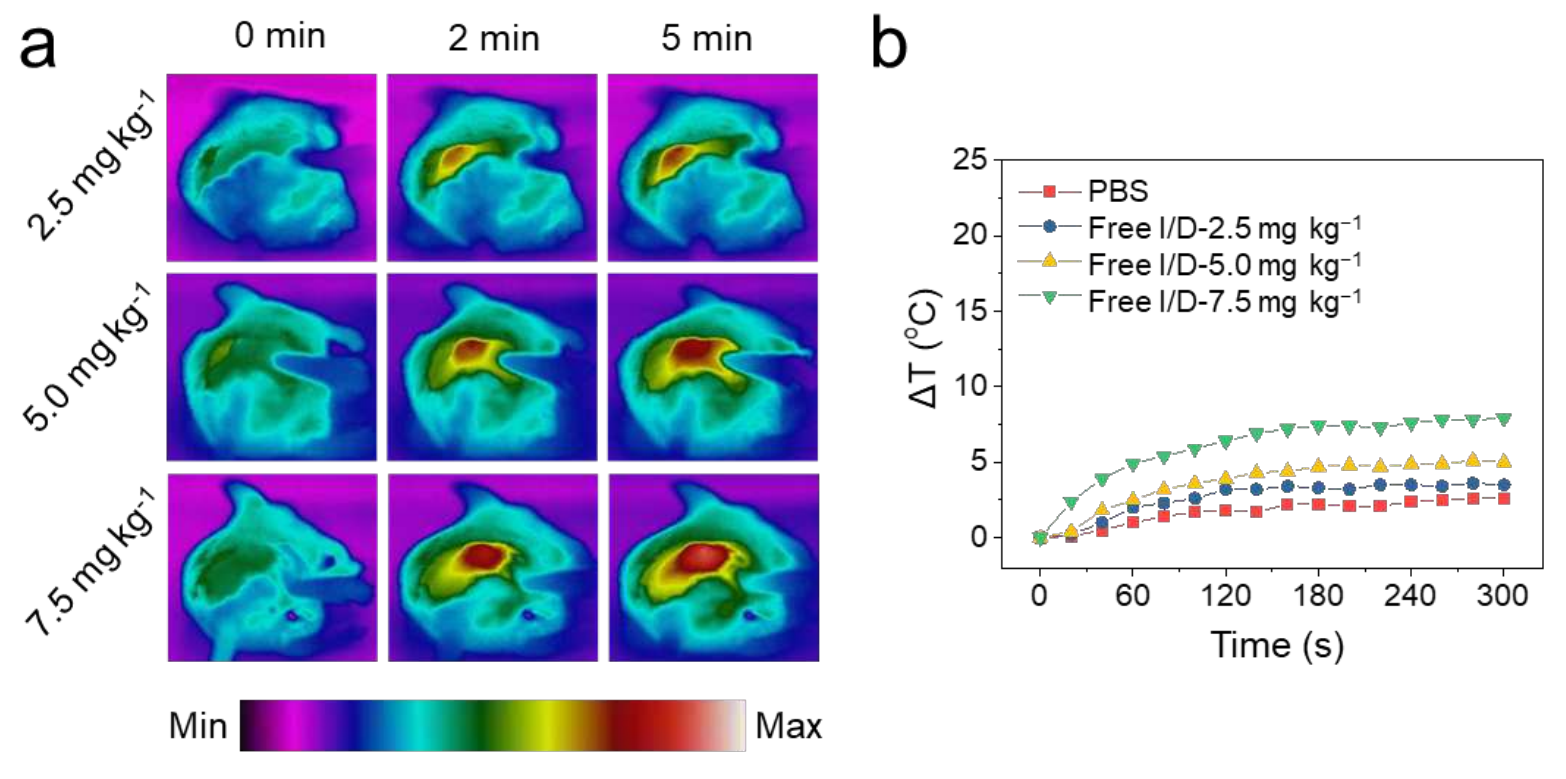

901

902 Supplementary Fig. 20. Infrared thermography of the mice bearing 4T1 subcutaneous tumours

903 treating with Free I/D at different doses of ICG (a) and their temperature elevations (b).

904 


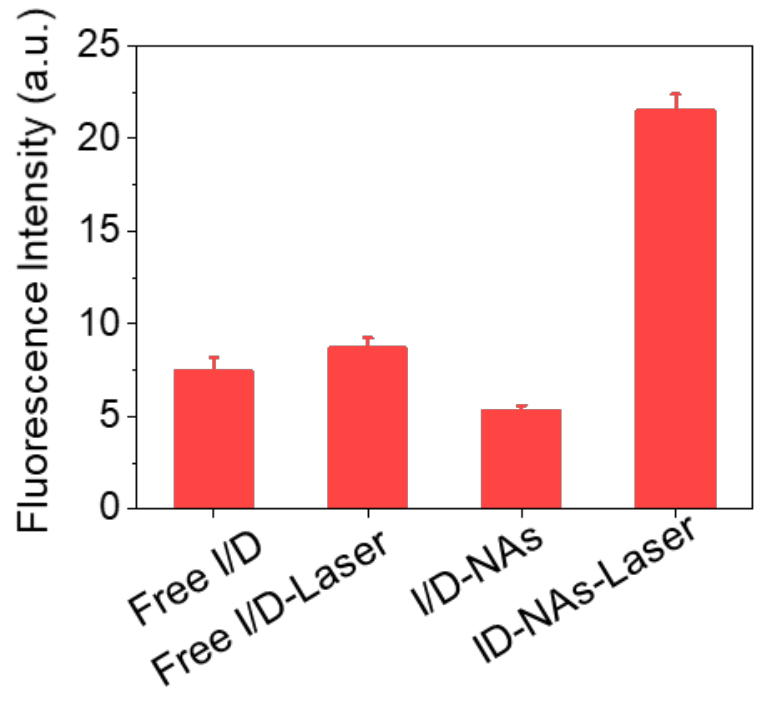

906 Supplementary Fig. 21. Fluorescence intensities calculated from DHE stained tumour sections of

907 4T1 subcutaneous tumour bearing mice treated with Free I/D and I/D-NAs at the dose of $7.5 \mathrm{mg}$ $908 \mathrm{~kg}^{-1}$ ICG after 3 min irradiation $\left(785 \mathrm{~nm}, 1.5 \mathrm{~W} \mathrm{~cm}^{-2}\right)$ or not. 

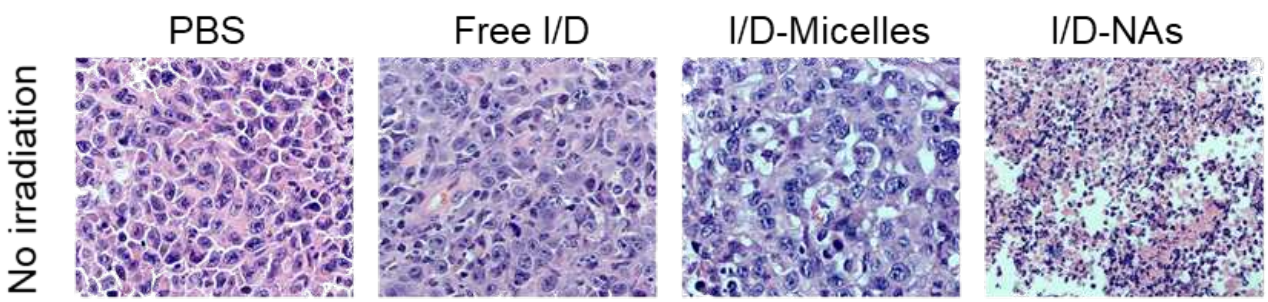

ICG-NAs
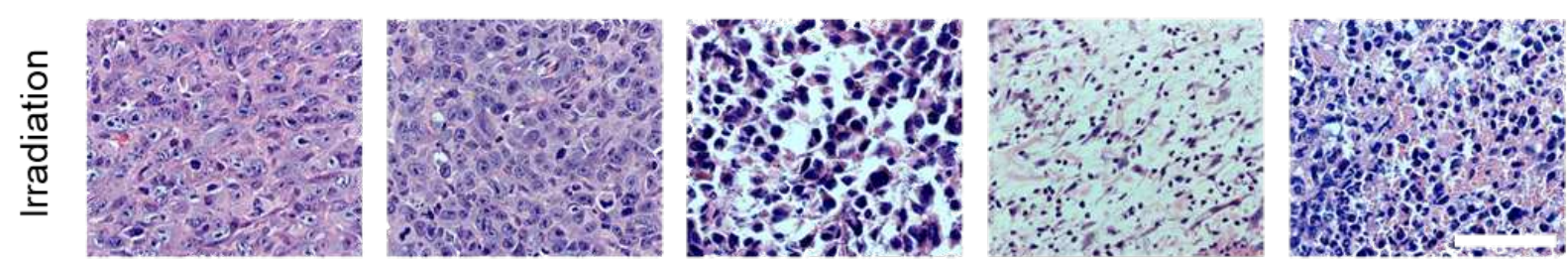

909

910 Supplementary Fig. 22. Images of H\&E stained tumour sections from the mice bearing

911 subcutaneous 4T1 tumour which suffered from various formulations at the dose of $7.5 \mathrm{mg} \mathrm{kg}^{-1}$

912 ICG under 3 min irradiation $\left(785 \mathrm{~nm}, 1.5 \mathrm{~W} \mathrm{~cm}^{-2}\right.$ ) or not at $24 \mathrm{~h}$ post-injection (scale bar: $\left.50 \mu \mathrm{m}\right)$. 


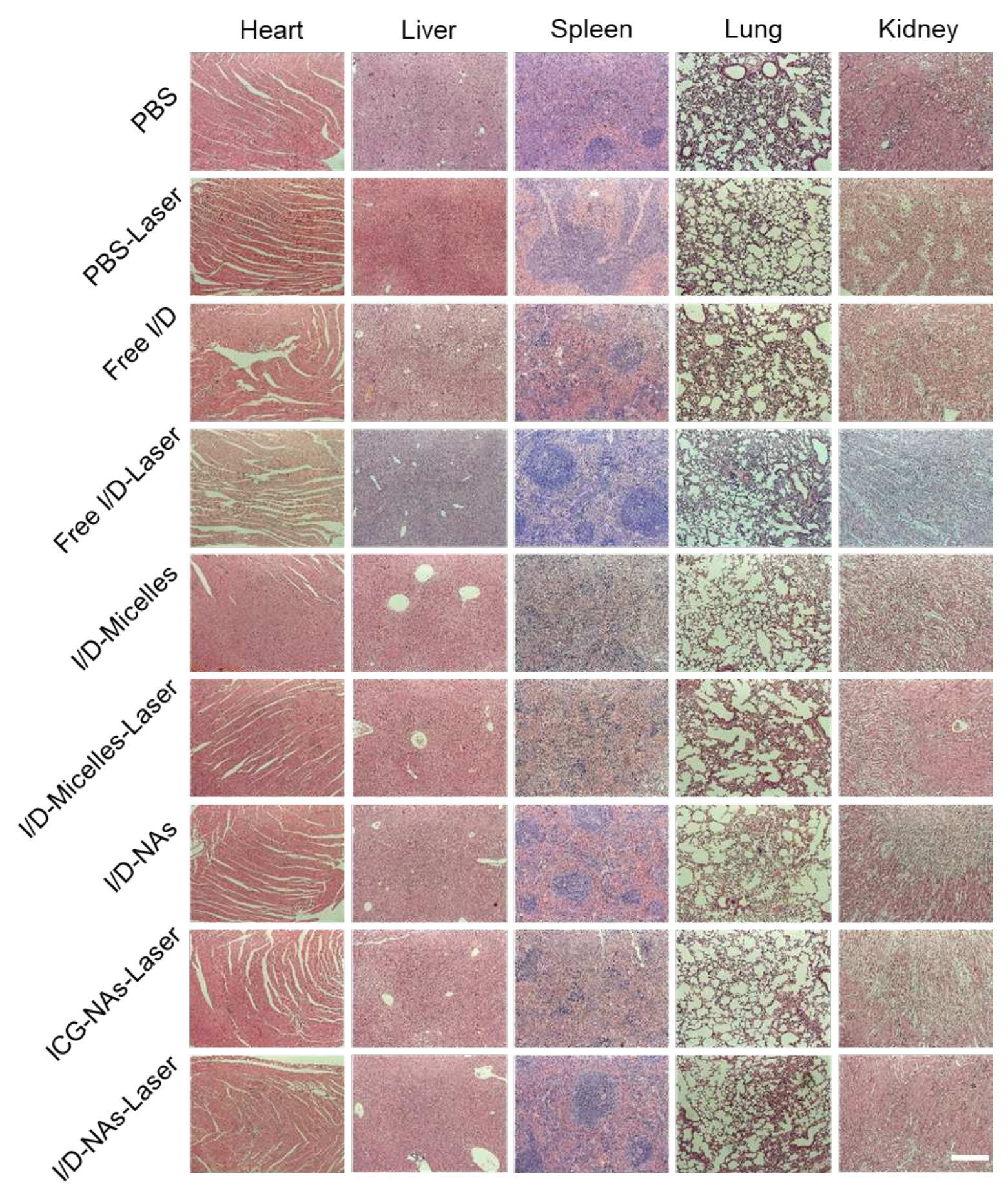

914

915 Supplementary Fig. 23. Images of H\&E-stained sections of heart, liver, spleen, lung, and kidney,

916 harvested from the mice bearing 4T1 subcutaneous tumours treated with different formulations at

917 the dose of $7.5 \mathrm{mg} \mathrm{kg}^{-1}$ ICG (scale bar: $200 \mu \mathrm{m}$ ).

918 


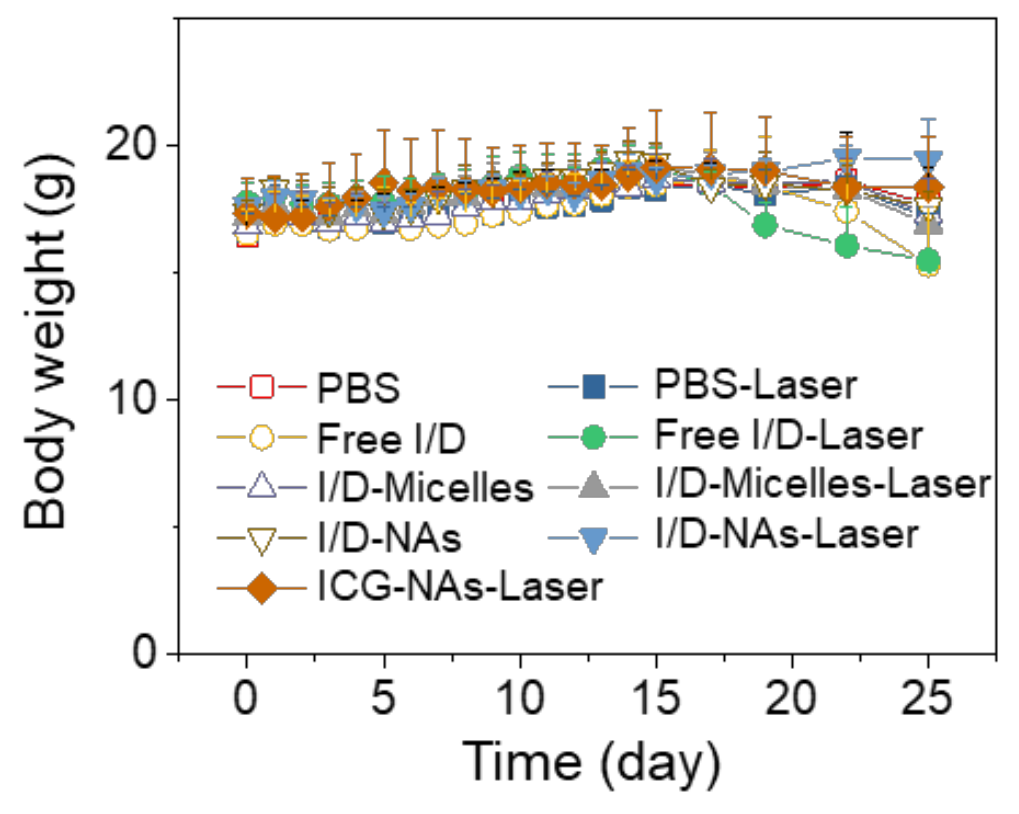

919

920 Supplementary Fig. 24. Body weights of the mice bearing 4T1 subcutaneous tumours treated

921 with Free I/D, I/D-Micelles, ID-NAs and ICG-NAs at the dose of $7.5 \mathrm{mg} \mathrm{kg}^{-1}$ ICG with light 922 irradiation or not during 25 days.

923 


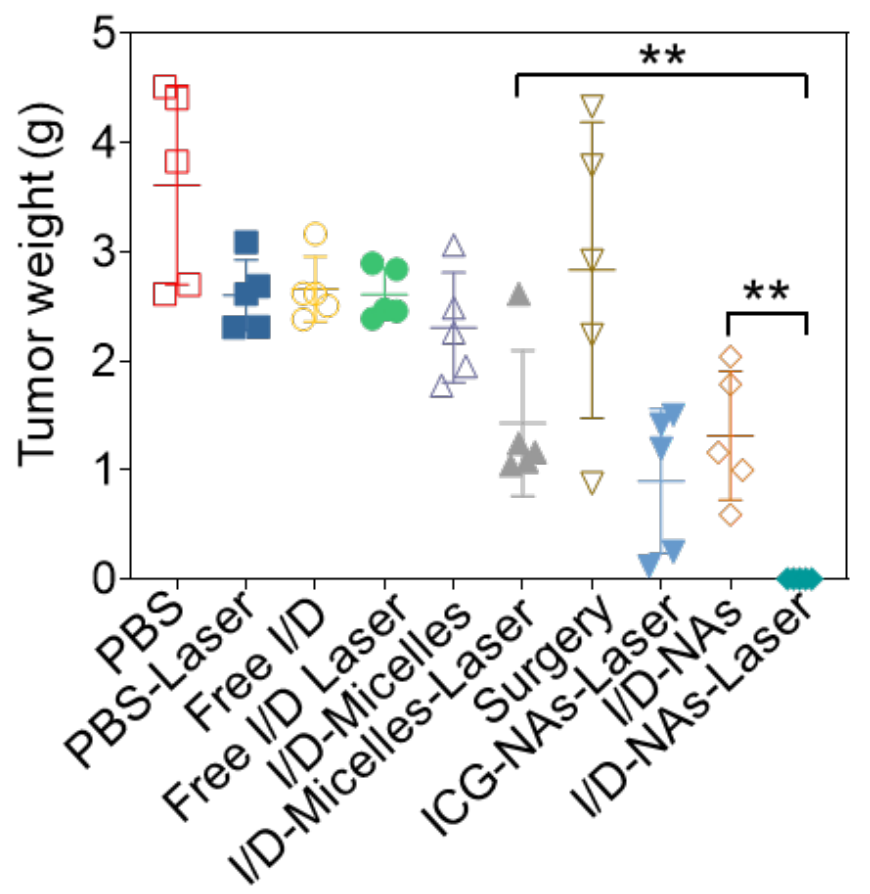

924

925 Supplementary Fig. 25. Tumour weights of the mice bearing orthotopic 4T1 breast tumours 926 treated with surgery, Free I/D, I/D-Micelles, I/D-NAs and ICG-NAs at the dose of $7.5 \mathrm{mg} \mathrm{kg}^{-1}$ ICG 927 under light irradiation or not at 20 days post-injection.

928 


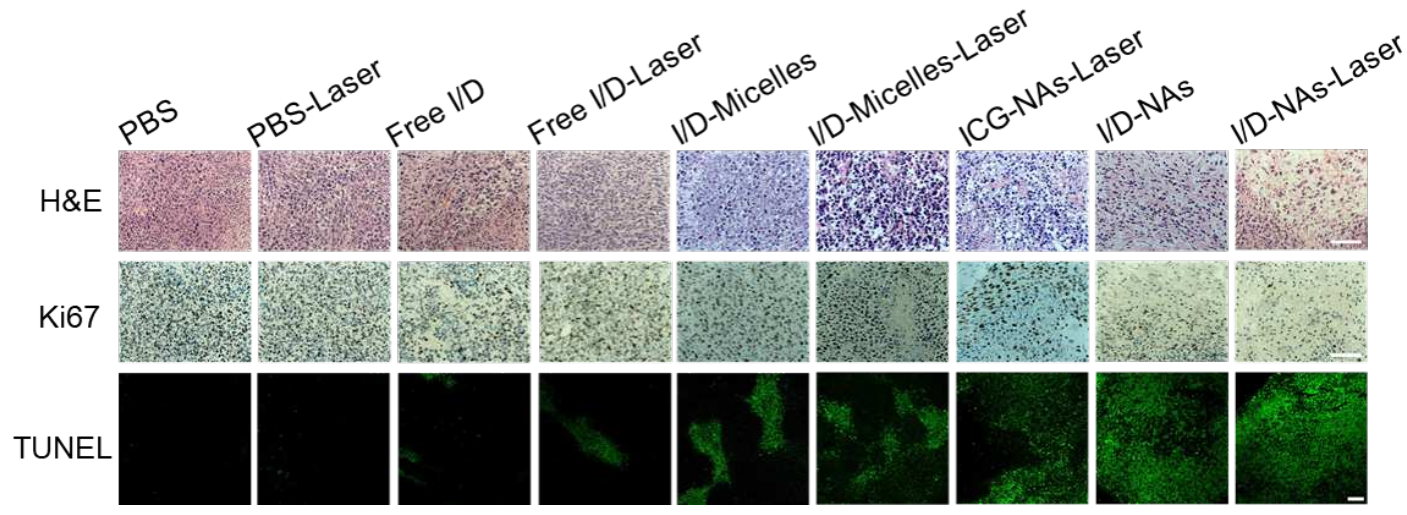

930 Supplementary Fig. 26. H\&E staining images (scale bar: $50 \mu \mathrm{m}$ ), Ki67 staining images (scale bar:

$93150 \mu \mathrm{m}$ ) and TUNEL staining images (scale bar: $100 \mu \mathrm{m}$ ) of tumour sections at $6 \mathrm{~h}$ post-irradiation. 


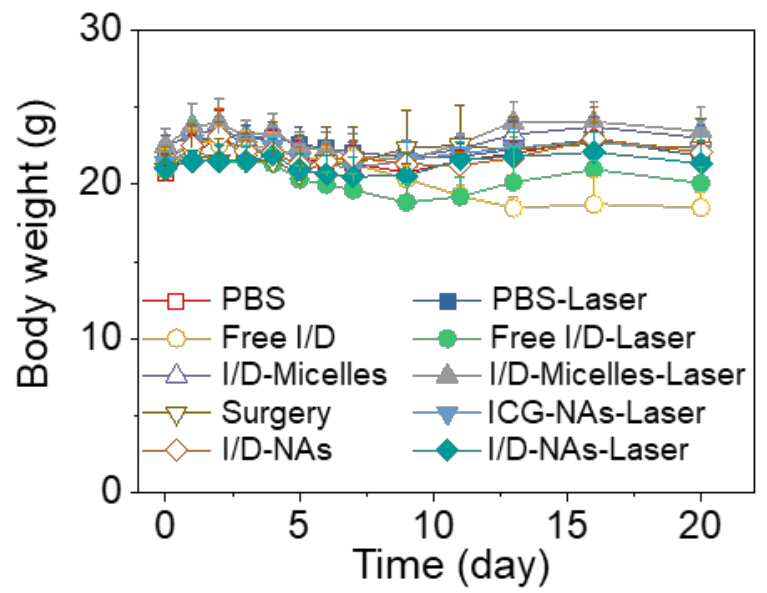

934 Supplementary Fig. 27. Body weights of mice bearing orthotopic 4T1 breast tumours after 935 treating with surgery, Free I/D, I/D-Micelles, I/D-NAs and ICG-NAs at the dose of $7.5 \mathrm{mg} \mathrm{kg}^{-1}$ 936 ICG with light irradiation or not during 20 days post-irradiation. 
938 Supplementary Table 1. Results for synthesis of disulfide-functionalized copolymers including 939 poly(VBHP-co-MTL) (S1), poly((St-PtBA)-co-MTL) (S2), poly((St-PtBA)-co-(MI-PNIPAM)) 940 (S3) and poly((St-PtBA/PCL)-co-(MI-PNIPAM/PCL)) (S4-S6) ${ }^{a}$

\begin{tabular}{ccccccccc}
\hline run & sample & $\mathrm{CTA} / \mathrm{I}^{b}$ & $\mathrm{M}$ & $\mathrm{C}^{c}$ & $M_{\mathrm{n}, \mathrm{th}}(\mathrm{kDa})^{d}$ & $M_{\mathrm{n}, \mathrm{GPC}}(\mathrm{kDa})^{e}$ & $\mathrm{Ð}^{e}$ & $M_{\mathrm{n}, \mathrm{NMR}}(\mathrm{kDa})^{f}$ \\
\hline 1 & $\mathrm{~S} 1$ & $\mathrm{~S}-\mathrm{CPDB}$ & $\mathrm{VBHP} / \mathrm{MTL}$ & 69.6 & 8.98 & 8.24 & 1.28 & 9.03 \\
2 & $\mathrm{~S} 2$ & $\mathrm{~S} 1$ & $t \mathrm{BA}$ & 4.32 & 32.3 & 18.6 & 1.26 & 33.2 \\
3 & $\mathrm{~S} 3$ & $\mathrm{~S} 2$ & $\mathrm{NIPAM}$ & 84.8 & 73.5 & 31.2 & 1.86 & 72.8 \\
4 & $\mathrm{~S} 4$ & $\mathrm{~S} 3$ & $\mathrm{CL}$ & 75.0 & 109 & 35.6 & 1.90 & 108 \\
5 & $\mathrm{~S} 5$ & $\mathrm{~S} 3$ & $\mathrm{CL}$ & 73.2 & 131 & 44.3 & 1.88 & 130 \\
6 & $\mathrm{~S} 6$ & $\mathrm{~S} 3$ & $\mathrm{CL}$ & 68.9 & 183 & 59.4 & 1.92 & 185 \\
\hline
\end{tabular}

$941{ }^{a}$ Reaction conditions: [VBHP $]_{0}:[\mathrm{MTL}]_{0}:[\mathrm{S}-\mathrm{CPDB}]_{0}:[\mathrm{AIBN}]_{0}=20: 20: 1: 0.3,[\mathrm{M}]_{0}=1.0 \mathrm{~mol} \mathrm{~L}^{-1}$, 942 in dioxane at $70{ }^{\circ} \mathrm{C}$ for $20 \mathrm{~h}$ (run 1); $[t \mathrm{BA}]_{0}:[-\mathrm{Br}]_{0}:[\mathrm{CuBr}]_{0}:[\mathrm{PMDETA}]_{0}=300: 1: 0.5: 0.5,[\mathrm{M}]_{0}=$ $9433.0 \mathrm{~mol} \mathrm{~L}^{-1}$, in dioxane at $60{ }^{\circ} \mathrm{C}$ for $15 \mathrm{~h}$, followed by end-capping reaction to deactivate ATRP 944 and RAFT moieties (run 2); [thiolactone $]_{0}:\left[\mathrm{HOCH}_{2} \mathrm{CH}_{2} \mathrm{NH}_{2}\right]_{0}:[\mathrm{NIPAM}]_{0}:[\mathrm{AIBN}]_{0}=1: 2: 30: 0.01$, $945[\mathrm{M}]_{0}=1.0 \mathrm{~mol} \mathrm{~L}{ }^{-1}$, in DMF at $25^{\circ} \mathrm{C}$ for $20 \mathrm{~h}$ and $65^{\circ} \mathrm{C}$ for $24 \mathrm{~h}$ (run 3); [CL $]_{0}:[-\mathrm{OH}]_{0}:\left[\mathrm{Sn}(\mathrm{Oct})_{2}\right]_{0}$ $946=x: 1: 0.2, x=15\left(\right.$ run 4), 25 (run 5) and $50\left(\right.$ run 6), $[\mathrm{M}]_{0}=1.2 \mathrm{~mol} \mathrm{~L}^{-1}$, in toluene at $100{ }^{\circ} \mathrm{C}$ for 20 $947 \mathrm{~h}$ (runs 4-6). ${ }^{b}$ Chain transfer agent (CTA, run 1) or macroinitiator (I, other runs). ${ }^{c}$ Monomer 948 conversion determined by gravimetry. ${ }^{d}$ Theoretical molar mass. ${ }^{e}$ Apparent molar mass $\left(M_{\mathrm{n}, \mathrm{GPC}}\right)$ 949 and dispersity (Đ) estimated by DMF GPC. ${ }^{f}$ Molar mass determined by ${ }^{1} \mathrm{H}$ NMR analysis. 
951 Supplementary Table 2. Photophysical properties of I/D-NAs and Free I/D.

\begin{tabular}{lll}
\hline Parameter & Free I/D & I/D-NAs \\
\hline ROS quantum yield & 0.14 & 0.21 \\
Fluorescence quantum yield & $0.5 \%$ & $0.15 \%$ \\
Photothermal conversion efficiency & $20.3 \%$ & $24.6 \%$ \\
\hline
\end{tabular}

952 
953 Supplementary Table 3. Pharmacokinetic parameters in plasma for DOX in BALB/c mice at the 954 dose of $7.5 \mathrm{mg} \mathrm{kg}^{-1}$ ICG equivalent of Free I/D and I/D-NAs.

\begin{tabular}{ccc}
\hline Parameter & Free I/D & I/D-NAs \\
\hline $\mathrm{T}_{1 / 2 \alpha}(\mathrm{h})$ & 0.1 & 0.1 \\
$\mathrm{~T}_{1 / 2 \beta}(\mathrm{h})$ & 1.2 & 5.2 \\
$\mathrm{AUC}\left(\mathrm{mg} \cdot \mathrm{h} \cdot \mathrm{L}^{-1}\right)$ & 20.7 & 60.8 \\
$\mathrm{CL}\left(\mathrm{L} \cdot \mathrm{h} \cdot \mathrm{kg}^{-1}\right)$ & 3.4 & 0.5 \\
\hline
\end{tabular}

955 
956 Supplementary Table 4. Plasma pharmacokinetic parameters of ICG from Free I/D and I/D957 NAs in the BALB/c mice at the dose of $7.5 \mathrm{mg} \mathrm{kg}^{-1}$ ICG.

\begin{tabular}{ccc}
\hline Parameter & Free I/D & I/D-NAs \\
\hline $\mathrm{T}_{1 / 2 \alpha}(\mathrm{h})$ & 0.1 & 0.1 \\
$\mathrm{~T}_{1 / 2 \beta}(\mathrm{h})$ & 0.8 & 4.9 \\
$\mathrm{AUC}\left(\mathrm{mg} \cdot \mathrm{h} \cdot \mathrm{L}^{-1}\right)$ & 10.5 & 90.2 \\
$\mathrm{CL}\left(\mathrm{L} \cdot \mathrm{h} \cdot \mathrm{kg}^{-1}\right)$ & 3.9 & 0.3 \\
\hline
\end{tabular}

958 


\section{Supplementary Methods}

960 Materials. The chemicals were ordered from Sigma-Aldrich unless otherwise stated. 2,2'961 Azobis(isobutyronitrile) (AIBN, 99\%) was recrystallized twice from ethanol, $\varepsilon$-caprolactone (CL, 962 99\%) was dried over $\mathrm{CaH}_{2}$ and distilled under reduced pressure, tert-butyl acrylate $(t \mathrm{BA}, 99 \%$, 963 Alfa-Aesar) was passed through a basic alumina column to remove the inhibitor, and $N$ 964 isopropylacrylamide (NIPAM, 97\%) was recrystallized twice from the mixtures of hexane and 965 toluene. $\mathrm{CuBr}(98 \%)$ was purified by stirring in acetic acid, washing with ethanol and drying under 966 vacuum conditions. 1-Butanethiol (98\%, 3a-Chemicals), dithiothreitol (DTT, 99\%, Merck), tris(2967 dimethylaminoethyl)amine (Me6TREN, 98\%, TCI), 4-vinylbenzyl chloride (VBC, 90\%, TCI), $968 D, L$-homocysteine thiolactone hydrochloride (98\%, Aladdin), $N, N^{\prime}$-dimethylethylenediamine 969 (DMDA, 99\%, Adamas), stannous octoate $\left(\mathrm{Sn}(\mathrm{Oct})_{2}, 97 \%\right), N, N^{\prime}$-dicyclohexylcarbodiimide (DCC, 970 95\%, Macklin), 4-dimethylamino pyridine (DMAP, 97\%, Macklin), 2-bromoisobutyric acid (98\%, 971 Macklin), and other reagents with analytical grade were used as received. Anisole, acetonitrile, 972 dichloromethane, tetrahydrofuran and 1,4-dioxane were purified using standard procedures. Bis(2973 hydroxyethyl)disulfide di(4-(benzodithioyl)-4-cyanopentanoate) (S-CPDB) ${ }^{1}$, 4-vinylbenzyl-3-(2974 bromo-2-methylpropanoyloxy)-2-hydroxymethyl-2-methylpropionate $\quad(\mathrm{VBHP})^{2}$ and 2975 maleimidyl-4-thiobutyrolactone (MTL) ${ }^{3}$ were synthesized according to the references.

Synthesis of poly((St-PtBA/PCL)-co-(MI-PNIPAM/PCL)) heterografted copolymers.

978 Starting from a disulfide-functionalized RAFT agent S-CPDB, controlled polymerization, 979 telomerization and selective hydrolysis were combined to achieve multi-responsive heterografted 980 copolymers H4-H6 comprising two types of V-shaped side chains. The terpolymers were 981 comprised of pH-responsive poly(acrylic acid) (PAA), thermoresponsive $\operatorname{poly}(N-$ 982 isopropylacrylamide) (PNIPAM) and biodegradable poly( $\varepsilon$-caprolactone). Detailed syntheses and 983 characterizations were described below.

984 Firstly, reversible addition-fragmentation chain transfer (RAFT) copolymerization of VBHP 985 with MTL were used to synthesize poly(VBHP-co-MTL) (S1) containing hydroxyl, alkyl bromide 986 and thiolactone functionalities. MTL (0.493 g, $2.50 \mathrm{mmol})$, S-CPDB (84.6 mg, $0.125 \mathrm{mmol})$, 987 VBHP (0.998 g, $2.50 \mathrm{mmol})$, and AIBN (6.2 mg, $0.038 \mathrm{mmol})$ were added to a Schlenk tube, 988 followed by addition of dry 1,4-dioxane to reach a total volume of $5.0 \mathrm{~mL}$. The contents were 989 degassed with bubbled nitrogen for 20 min and then subjected to polymerization at $70{ }^{\circ} \mathrm{C}$ for $20 \mathrm{~h}$. 
990 The polymerization solution was concentrated and repeatedly precipitated into diethyl ether, and 991 the monomer conversion was obtained as $69.6 \%$ by gravimetry. GPC analysis: $M_{\mathrm{n}, \mathrm{GPC}}=8240 \mathrm{Da}$, $992 Ð=1.28$. Based on ${ }^{1} \mathrm{H}$ NMR analysis, DP ${ }_{\text {MTL unit }}=4 I_{4.62} / I_{7.91}=14, \mathrm{DP}_{\mathrm{VBHP} \text { unit }}=2 I_{5.15} / I_{7.91}=14$ 993 (DP denotes number of monomer unit or degree of polymerization), and $M_{\mathrm{n}, \mathrm{NMR}}=9030 \mathrm{Da} .{ }^{1} \mathrm{H}$ $994 \mathrm{NMR}\left(\mathrm{CDCl}_{3}\right): \delta$ 6.0-8.0 (m, $\mathrm{PhH}$ and $\left.\mathrm{Ar} H\right), 5.15\left(\mathrm{~s}, \mathrm{ArCH}_{2} \mathrm{O}\right), 4.62(\mathrm{~m}, \mathrm{CONHCH}$ of MTL unit), $9954.38\left(\mathrm{~s}, \mathrm{CCH}_{2} \mathrm{O}\right.$ of VBHP unit and $\left.\mathrm{COOCH}_{2} \mathrm{CH}_{2} \mathrm{~S}\right), 3.73$ (s, $\mathrm{CH}_{2} \mathrm{OH}$ of VBHP unit), 3.32 (m, $996 \mathrm{CHCHCO}$ and $\mathrm{CH}_{2} \mathrm{~S}$ of MTL unit), $2.89\left(\mathrm{~m}, \mathrm{OCH}_{2} \mathrm{CH}_{2} \mathrm{~S}\right), 0.6-2.7\left(\mathrm{~m}\right.$, other $\mathrm{CH}, \mathrm{CH}_{2}$ and $\mathrm{CH}_{3}$ 997 from monomer units and linking group). FT-IR (ATR): 2928, 1697, 1517, 1452, 1391, 1276, 1203, $9981161,1104,1047,1008,932,844 \mathrm{~cm}^{-1}$.

999 Secondly, poly((St-PtBA)-co-MTL) (S2) was synthesized by combination of grafting-from 1000 strategy and end-capping reaction. S1 was initially used to initiate the atom transfer radical 1001 polymerization (ATRP) of $t \mathrm{BA}$. In a typical experiment, $\mathrm{S} 1(0.200 \mathrm{~g}, 0.310 \mathrm{mmol}$ of $\mathrm{Br}), t \mathrm{BA}(11.9$ $1002 \mathrm{~g}, 92.8 \mathrm{mmol}), \mathrm{CuBr}(22.3 \mathrm{mg}, 0.155 \mathrm{mmol})$, and PMDETA (26.9 mg, $0.155 \mathrm{mmol}$ ) were added 1003 to a round-bottom flask, followed by addition of dry 1,4-dioxane to reach a total volume of $31 \mathrm{~mL}$. 1004 The contents were degassed with bubbled nitrogen for $30 \mathrm{~min}$, and then the polymerization was 1005 performed at $60{ }^{\circ} \mathrm{C}$ for $15 \mathrm{~h}$. The polymerization solution was concentrated, followed by repeated 1006 precipitation into diethyl ether to isolate polymer. After vacuum drying, the resultant graft polymer 1007 with monomer conversion of $4.32 \%$ was obtained. On this basis, the alkyl bromide was deactivated 1008 by thio-bromide click reaction, and the RAFT moiety was removed by radical-induced reaction 1009 using excess AIBN. Polymer (0.664 g, $0.020 \mathrm{mmol})$, AIBN (6.6 mg, $0.40 \mathrm{mmol}$ ) and acetonitrile $1010(5.0 \mathrm{~mL})$ were added to a Schlenk tube under nitrogen, and the mixture was heated to $80{ }^{\circ} \mathrm{C}$ for 3 1011 h. After cooling down, triethylamine (TEA, $40.5 \mathrm{mg}, 0.40 \mathrm{mmol}$ ) and butanethiol (BuSH, $36.1 \mathrm{mg}$, $10120.40 \mathrm{mmol}$ ) were added to the solution under nitrogen, followed by stirring at room temperature 1013 for $24 \mathrm{~h}$. Afterwards, methyl acrylate $(34.4 \mathrm{mg}, 0.40 \mathrm{mmol})$ was injected into the solution to react 1014 with excess butanethiol. After centrifugation, the isolated solution was concentrated and 1015 precipiated into hexane to give S2. GPC analysis: $M_{\mathrm{n}, \mathrm{GPC}}=18600 \mathrm{Da}, Ð=1.26$. Based on ${ }^{1} \mathrm{H}$ NMR 1016 analysis, $\mathrm{DP}_{\mathrm{P} t \mathrm{BA}}=2 I_{1.45} /\left(9 I_{5.12}\right)=13.5$, and $M_{\mathrm{n}, \mathrm{NMR}}=33200 \mathrm{Da} .{ }^{1} \mathrm{H} \mathrm{NMR}\left(\mathrm{CDCl}_{3}\right): \delta 6.0-7.5(\mathrm{~m}$, $1017 \mathrm{ArH}), 5.12\left(\mathrm{~s}, \mathrm{ArCH}_{2} \mathrm{O}\right), 4.63$ (m, CONHCH of MTL unit), 3.95-4.40 (m, $\left.\mathrm{COOCH}_{2}\right), 3.66$ (m, $1018 \mathrm{CH}_{2} \mathrm{OH}$ ), 3.32 (m, $\mathrm{CHCHCO}$ and $\mathrm{CH}_{2} \mathrm{~S}$ of MTL unit), 2.88 (m, $\left.\mathrm{OCH}_{2} \mathrm{CH}_{2} \mathrm{~S}\right), 0.6-2.7$ (m, other $1019 \mathrm{CH}, \mathrm{CH}_{2}$ and $\mathrm{CH}_{3}$ from polymer backbone, $\mathrm{CH}_{2} \mathrm{CH}_{2} \mathrm{~S}$ of MTL unit, linking and end groups, and 1020 PtBA grafts). FT-IR (ATR): 3283, 2976, 2934, 2876, 2737, 2675, 2491, 1718, 1437, 1457, 1393, 
1021

1022

1023

1024

1025

1026

1027

1028

1029

1030

1031

1032

1033

1034

1035

1036

1037

1038

1039

1040

1041

1042

1043

1044

1045

1046

1047

1048

1049

1050

1051

$1366,1255,1144,1011,844 \mathrm{~cm}^{-1}$.

Thirdly, tandem amine-thiol-telomerization reactions were adopted to generate poly $((\mathrm{St}-\mathrm{P} t \mathrm{BA})-$ co-(MI-PNIPAM)) (S3). In a typical run, S2 (0.332 g, $0.14 \mathrm{mmol}$ of thiolactone unit) was dissolved in $2.0 \mathrm{~mL}$ of dry DMF with a magnetic stirring bar under nitrogen for $20 \mathrm{~min}$, and then ethanolamine $(17.1 \mathrm{mg}, 0.28 \mathrm{mmol})$ was added to perform aminolysis. After stirring at room temperature for $20 \mathrm{~h}$, the DMF solution $(2.0 \mathrm{~mL})$ containing NIPAM $(0.475 \mathrm{~g}, 4.2 \mathrm{mmol})$ and AIBN $(0.23 \mathrm{mg}, 1.4 \mu \mathrm{mol})$ were injected into the solution under nitrogen. The reaction was conducted at $65{ }^{\circ} \mathrm{C}$ for $24 \mathrm{~h}$, and S2 with monomer conversion of $84.8 \%$ was obtained after purification. GPC analysis: $M_{\mathrm{n}, \mathrm{GPC}}=31200 \mathrm{Da}, \emptyset=1.86$. Based on ${ }^{1} \mathrm{H}$ NMR analysis, DPPNIPAM $=I_{1.14} /\left(3 I_{5.12}\right)=25.0$, and $M_{\mathrm{n}, \mathrm{NMR}}=72800 \mathrm{Da} .{ }^{1} \mathrm{H} \mathrm{NMR}\left(\mathrm{CDCl}_{3}\right): \delta 6.0-7.5(\mathrm{~m}, \mathrm{Ar} H$ and $\mathrm{CON} H$ of PNIPAM), 5.42 (m, NCHCO), 5.14 (m, $\mathrm{ArCH}_{2} \mathrm{O}$ ), 3.85-4.40 (m, $\mathrm{COOCH}_{2}$ and $\mathrm{CHNH}$ of PNIPAM), 3.75 (m, $\left.\mathrm{NHCH}_{2} \mathrm{CH}_{2} \mathrm{OH}\right), 3.66$ (m, $\left.\mathrm{CCH}_{2} \mathrm{OH}\right), 3.15-3.50$ (m, $\mathrm{NHCH}_{2} \mathrm{CH}_{2} \mathrm{OH}$, $\mathrm{CH}_{2} \mathrm{CHS}$, and $\mathrm{CHCHCO}$ ), 2.5-3.1 (m, $\left.\mathrm{CH}_{2} \mathrm{~S}\right), 0.6-2.4$ (m, other $\mathrm{CH}, \mathrm{CH}_{2}$ and $\mathrm{CH}_{3}$ from polymer backbone, linking and end groups, and PtBA and PNIPAM grafts). FT-IR (ATR): 3277, 3075, 2973, $2933,1771,1725,1636,1542,1457,1388,1366,1255,1148,1064,1015,926,843 \mathrm{~cm}^{-1}$.

Fourthly, three examples of poly((St-PtBA/PCL)-co-(MI-PNIPAM/PCL)) (S4-S6) copolymers with different chain lengths of PCL grafts were synthesized by ring-opening polymerization (ROP) using S3 as a macroinitiator. In a typical run, $\mathrm{S} 3(0.208 \mathrm{~g}, 0.040 \mathrm{mmol}$ of $\mathrm{OH}), \mathrm{Sn}(\mathrm{Oct})_{2}(3.2 \mathrm{mg}$, $0.008 \mathrm{mmol})$, and CL (68.5 mg, $0.60 \mathrm{mmol})$ were added to a Schlenk tube under nitrogen, followed by addition of dry toluene to reach a total volume of $3.0 \mathrm{~mL}$. After degassing, the contents were subjected to polymerization at $100{ }^{\circ} \mathrm{C}$ for $20 \mathrm{~h}$. The product was purified by precipitation into hexane, and S4 was obtained in $75.0 \%$ of monomer conversion. GPC analysis: $M_{\mathrm{n}, \mathrm{GPC}}=35600 \mathrm{Da}$, $Ð=1.90$. Based on ${ }^{1} \mathrm{H}$ NMR analysis, $\mathrm{DP}_{\mathrm{PCL}}=I_{2.31} /\left(I_{4.48} \times \mathrm{DP}_{\text {backbone }}\right)=11$, and $M_{\mathrm{n}, \mathrm{NMR}}=108 \mathrm{kDa}$. According to similar procedures, S5 and S6 with longer chain length of PCL segment were prepared. ${ }^{1} \mathrm{H}$ NMR $\left(\mathrm{CDCl}_{3}\right): \delta$ 5.6-7.5 (m, $\mathrm{ArH}$ and $\mathrm{CONH}$ of PNIPAM), $5.10\left(\mathrm{~m}, \mathrm{ArCH} \mathrm{H}_{2} \mathrm{O}\right), 4.48$ (m, $\mathrm{OCH}_{2} \mathrm{CH}_{2} \mathrm{~S}$ ), 3.9-4.4 (m, $\mathrm{COOCH}_{2}, \mathrm{CHNH}$ of PNIPAM, and $\mathrm{CH}_{2} \mathrm{O}$ of PCL), 3.1-3.8 (m, CHCHCO, terminal $\mathrm{CH}_{2} \mathrm{OH}, \mathrm{NHCH}_{2} \mathrm{CH}_{2} \mathrm{O}$, and $\mathrm{CH}_{2} \mathrm{CHS}$ ), 0.6-3.0 (m, other $\mathrm{CH}, \mathrm{CH}_{2}$ and $\mathrm{CH}_{3}$ from polymer backbone, linking and end groups, and PtBA, PNIPAM and PCL grafts). FT-IR (ATR): 3285, 3074, 2970, 2935, 2869, 1724, 1644, 1543, 1458, 1389, 1366, 1243, 1151, 1104, $1048,844,732 \mathrm{~cm}^{-1}$.

Lastly, three examples of amphiphilic poly((St-PAA/PCL)-co-(MI-PNIPAM/PCL)) (H4-H6) 
1052 copolymers were generated by selective hydrolysis reaction of $\mathrm{P} t \mathrm{BA}$ segments. In a typical

1053 experiment, S4 $(200 \mathrm{mg}), \mathrm{DCM}(2.0 \mathrm{~mL})$, and trifluoroacetic acid $(120 \mathrm{mg})$ were successively 1054 added to a glass tube with a magnetic stirring bar, and the mixture was stirred at room temperature 1055 for $24 \mathrm{~h}$. After concentration and precipitation into hexane, $\mathrm{H} 4$ was obtained in $98.0 \%$ of yield. 1056 Starting from S5 and S6, H5 and H6 were synthesized using similar procedures. FT-IR (ATR): $10573365,2937,2875,1781,1708,1636,1547,1456,1391,1368,1207,1146,805,775,694 \mathrm{~cm}^{-1}$. 1058

1059 Characterization. Apparent molar mass $\left(M_{\mathrm{n}, \mathrm{GPC}}\right)$ and dispersity ( $\Theta$ or $\left.M_{\mathrm{w}} / M_{\mathrm{n}}\right)$ of various polymers 1060 were measured on a Waters 1515 gel permeation chromatography (GPC) using three MZ-Gel 1061 SDplus columns at $40{ }^{\circ} \mathrm{C}$, where the eluent and standard samples were DMF and PMMA, 1062 respectively. ${ }^{1} \mathrm{H}$ NMR spectra $(400 \mathrm{MHz})$ were measured on a Varian spectrometer at $25^{\circ} \mathrm{C}$ using $1063 \mathrm{CDCl}_{3}$. Fourier Transform Infrared (FT-IR) spectra of normal samples were measured on a Bruker 1064 Vertex 70 spectrometer. To determine the cloud point of various solutions, turbidity analysis was 1065 performed at $500 \mathrm{~nm}$ on a Shimadzu UV-3150 UV-vis spectrophotometer equipped with a 1066 thermoregulator.

1067 


\section{3. Supplementary References}

1069 1. Li, S., et al. Synthesis and properties of monocleavable amphiphilic comblike copolymers with 1070 alternating PEG and PCL grafts. J. Polym. Sci., Part A: Polym. Chem. 50, 3135-3148 (2012).

1071 2. Dai, W., et al. Temperature and solvent isotope dependent hierarchical self-assembly of a 1072 heterografted block copolymer. Chem. Commun. 55, 5709-5712 (2019).

1073 3. Rudolph, T., et al. Poly(thiolactone) homo- and copolymers from maleimide thiolactone: 1074 synthesis and functionalization. Polym. Chem. 6, 4240-4251 (2015). 


\section{Figures}

a

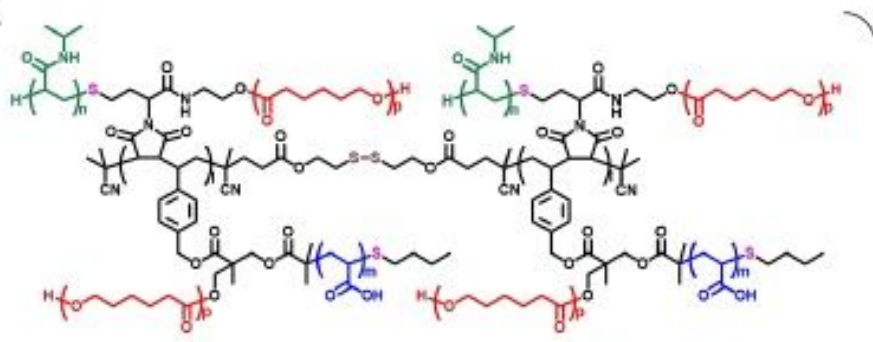

poly((St-PAA/PCL)-co-(MI-PNIPAM/PCL))

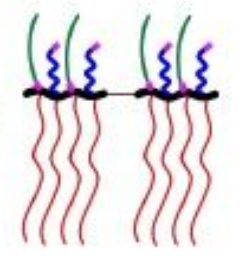

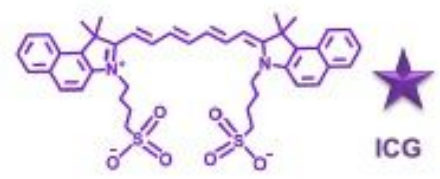

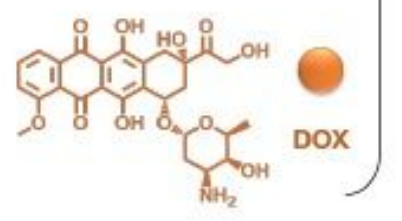

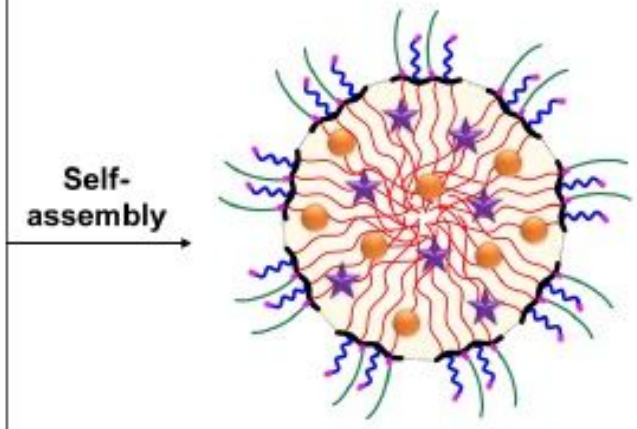

I/D-NAs

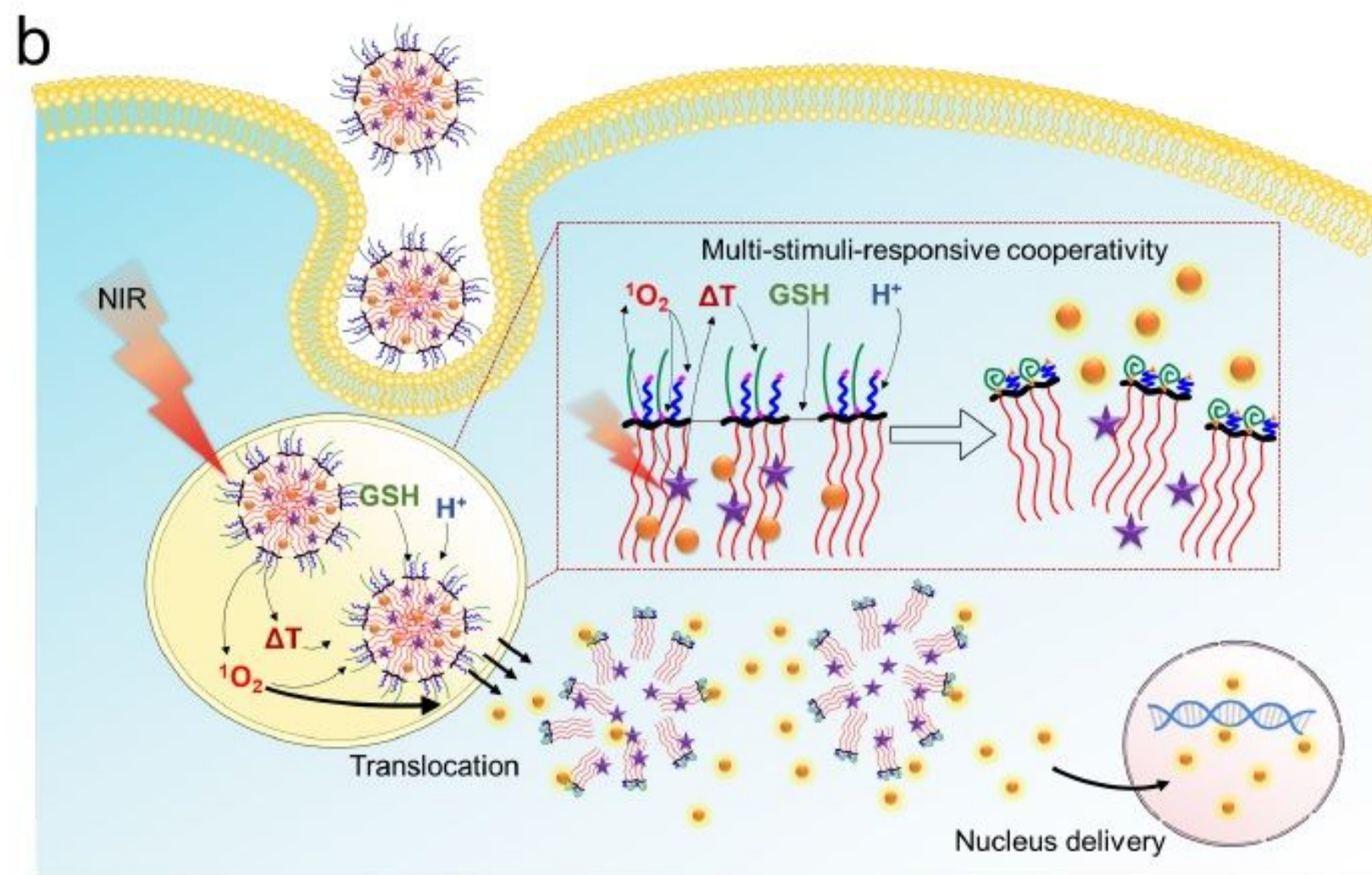

Figure 1

Schematic illustration of nanoassembly for anticancer therapy. a. Construction of $\mathrm{H}$ shaped miktobrush copolymer nanoassemblies with $\mathrm{pH}$ /reduction/temperature/ROS-responsive properties for coencapsulating representative antitumour compounds such as indocyanine green (ICG) and doxorubicin 
(DOX). b. Synergistic photo-chemotherapeutic mechanism against highly aggressive TNBC tumours through multi-stimuli-responsive drug release and ultrafast nucleus delivery.

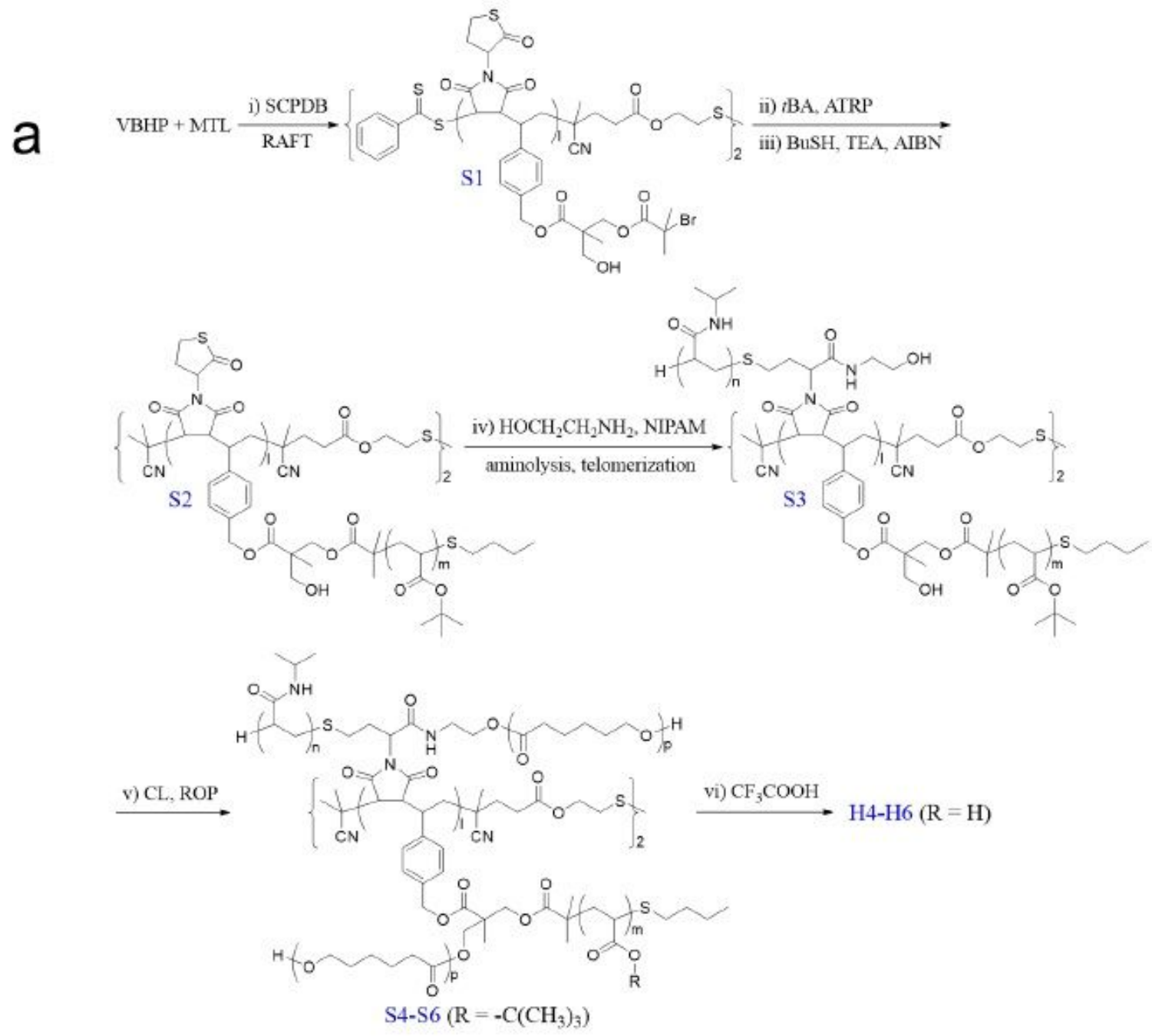

b

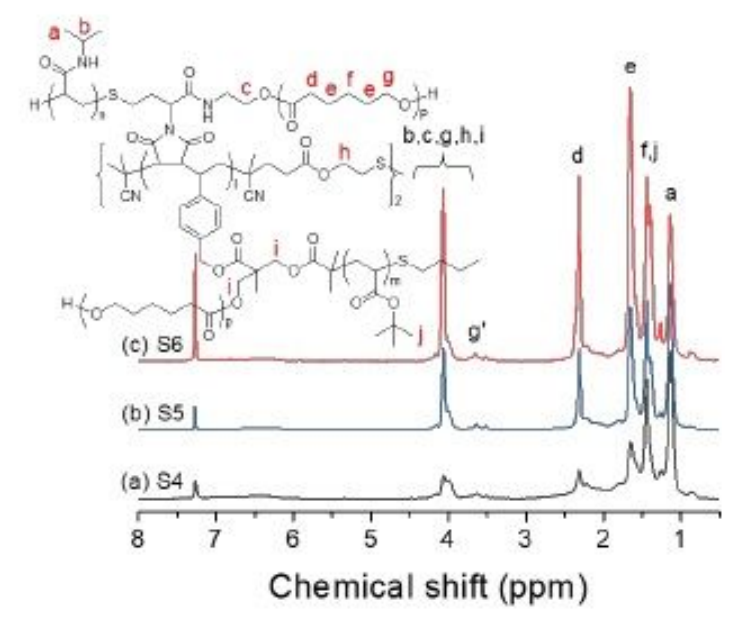

C

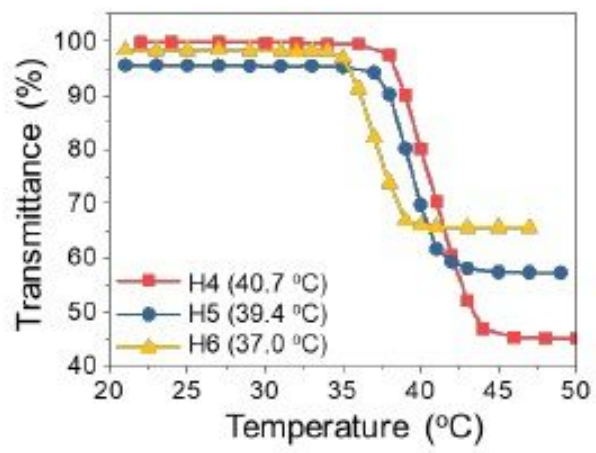

Figure 2

Synthesis and characterization of brush-like copolymers. a. Synthesis of disulfide bridged $\mathrm{pH} /$ reduction/temperature/ROS-responsive poly((St-PtBA/PCL)-co-(MI-PNIPAM/PCL) copolymers (H4-H6) by the combination of controlled polymerization, amine-734 thiol-telomerization and selective hydrolysis, 
in which $\mathrm{I} \approx 7, \mathrm{~m} \approx 14, \mathrm{n} \approx 25$, and $\mathrm{p} \approx 11(\mathrm{~S} 4, \mathrm{H} 4), 18(\mathrm{~S} 5, \mathrm{H} 5)$ or $35(\mathrm{~S} 6, \mathrm{H} 6)$. b. $1 \mathrm{H}$ NMR spectra of poly((St-PtBA/PCL)-co-(MI-PNIPAM/PCL)) copolymers recorded in $\mathrm{CDCl} 3$ at $25^{\circ} \mathrm{C}$, where g' denotes terminal $\mathrm{CH} 2 \mathrm{OH}$ of PCL grafts. c. Influence of temperature and chain length of PCL on transmittances of copolymer nanoassemblies ( $c p=1.0 \mathrm{mg} \mathrm{mL}-1)$ formed from disulfide-bridged poly((St-PAA/PCL)-co-(MIPNIPAM/PCL)) in aqueous solution.
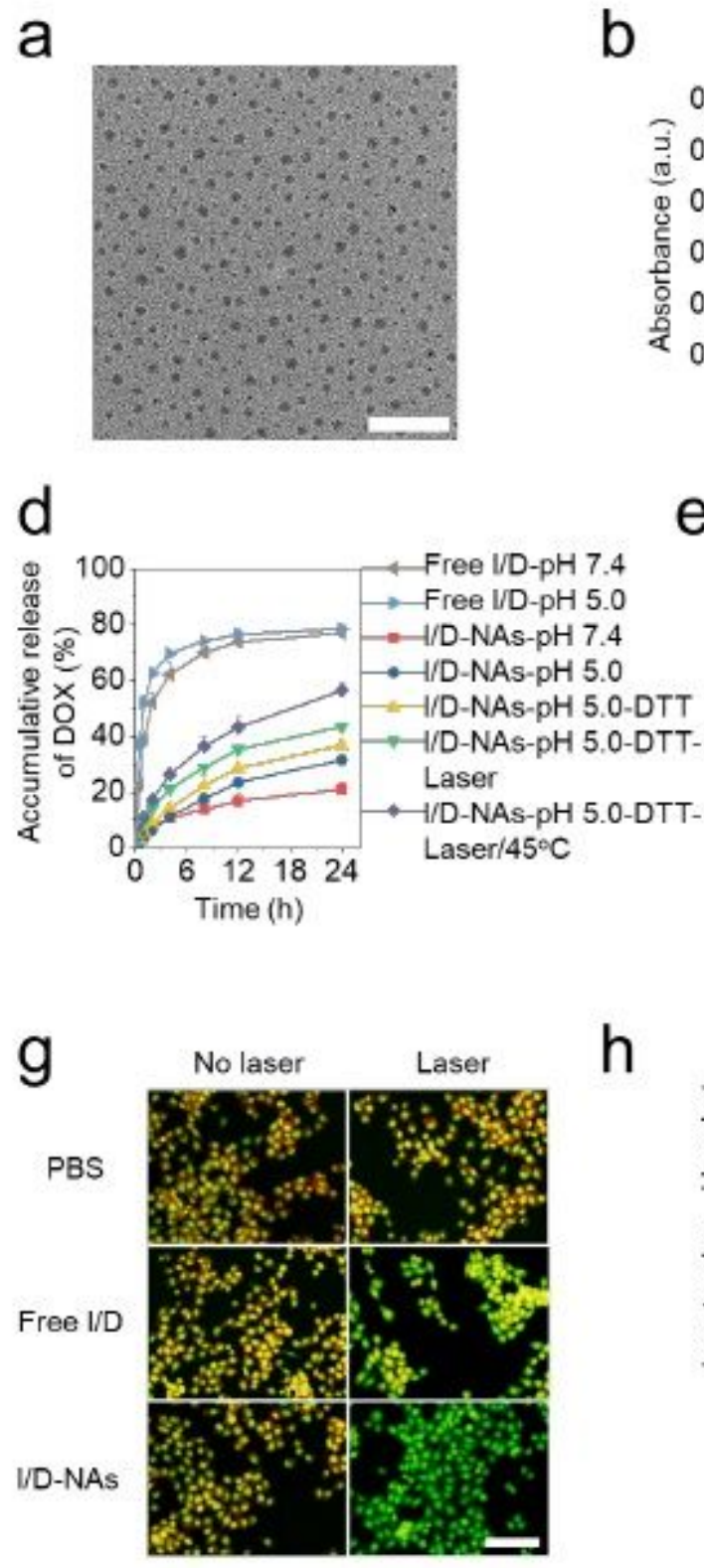

b

$\mathrm{h}$

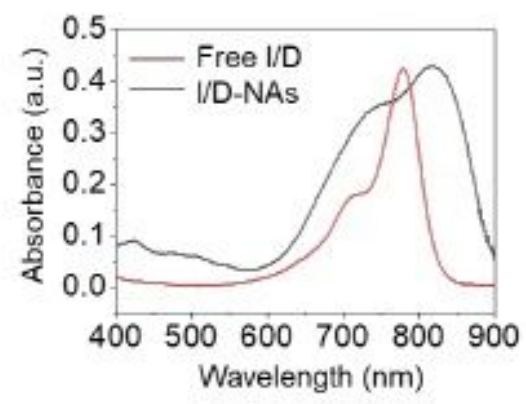

e
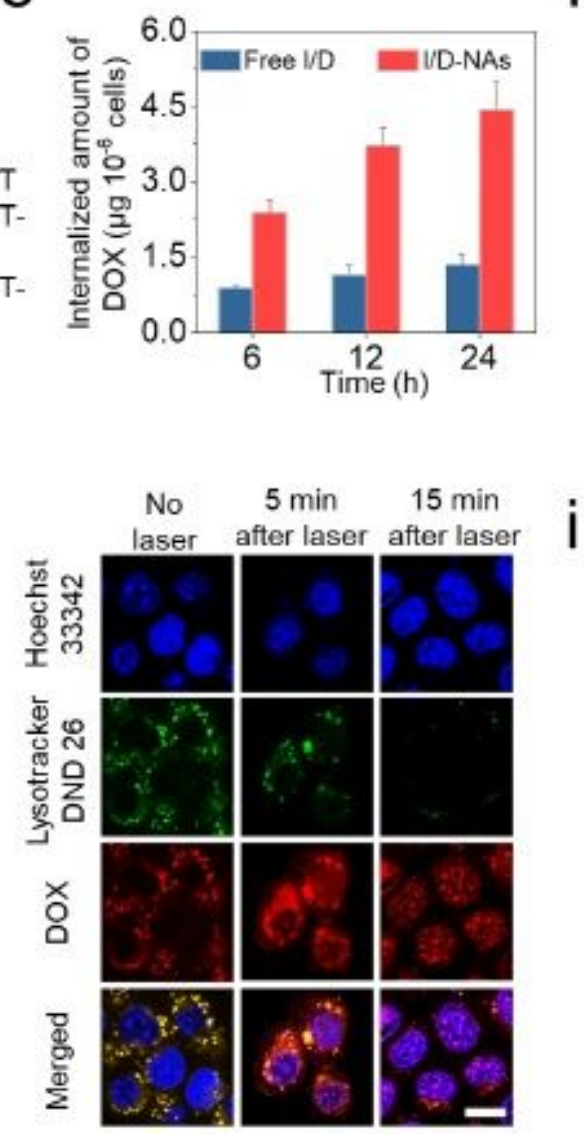

C

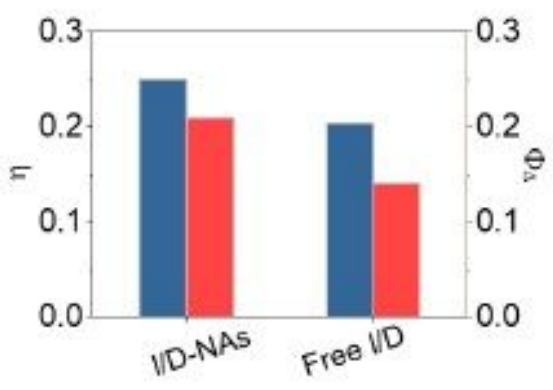

f
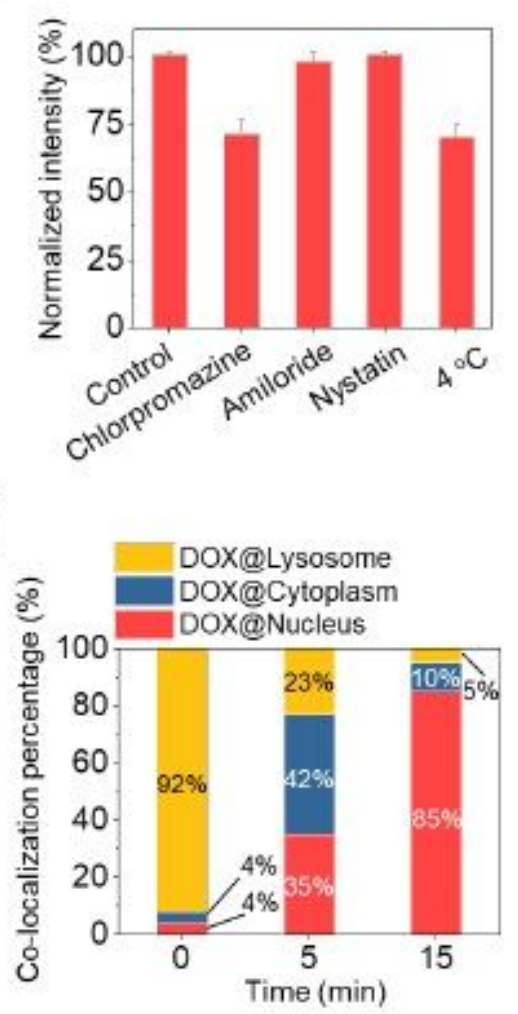

\section{Figure 3}

Enhanced lysosome-cytoplasma translocation and rapid nucleus delivery of I/D-NAs by multi-stimuliresponsive cooperativity. a. TEM image of I/D-NAs (scale bar: $500 \mathrm{~nm}$ ). b. UV-vis absorption of Free I/D and I/D-NAs. c. Photothermal conversion efficiency $(h)$ and Singlet oxygen quantum yield (FD) of Free I/D and I/D-NAs under $785 \mathrm{~nm}$ laser irradiation. d. Accumulative releases of DOX from Free I/D and I/D-NAs 
in various solutions. e. Internalized amounts of DOX in 4T1 cells treated by Free I/D and I/D-NAs at the dose of $10.0 \mu \mathrm{g} \mathrm{mL}-1 \mathrm{ICG}$ after 6,12 and $24 \mathrm{~h}$ incubation. $\mathrm{f}$. Cellular uptake by $4 \mathrm{~T} 1$ cells treated with various inhibitors. g.Lysosomal disruption of 4T1 cells treated with Free I/D and I/D-NAs 750 under irradiation or not (scale bar: $20 \mu \mathrm{m}$ ). h. Confocal laser scanning microscopy images (scale bar: $20 \mu \mathrm{m}$ ) of 4T1 cells stained with Lysotracker Green DND-26 and Hoechst 33342 after $2 \mathrm{~h}$ incubation with I/D-NAs at the dose of $10.0 \mu \mathrm{g} \mathrm{ml-1}$ ICG before irradiation, as well as 5 and $15 \mathrm{~min}$ post-irradiation ( $3 \mathrm{~min}, 1.5 \mathrm{~W}$ $\mathrm{cm}-2$ ), respectively. i. Co-localization percentage of DOX within different organelles as indicated in $\mathrm{h}$.
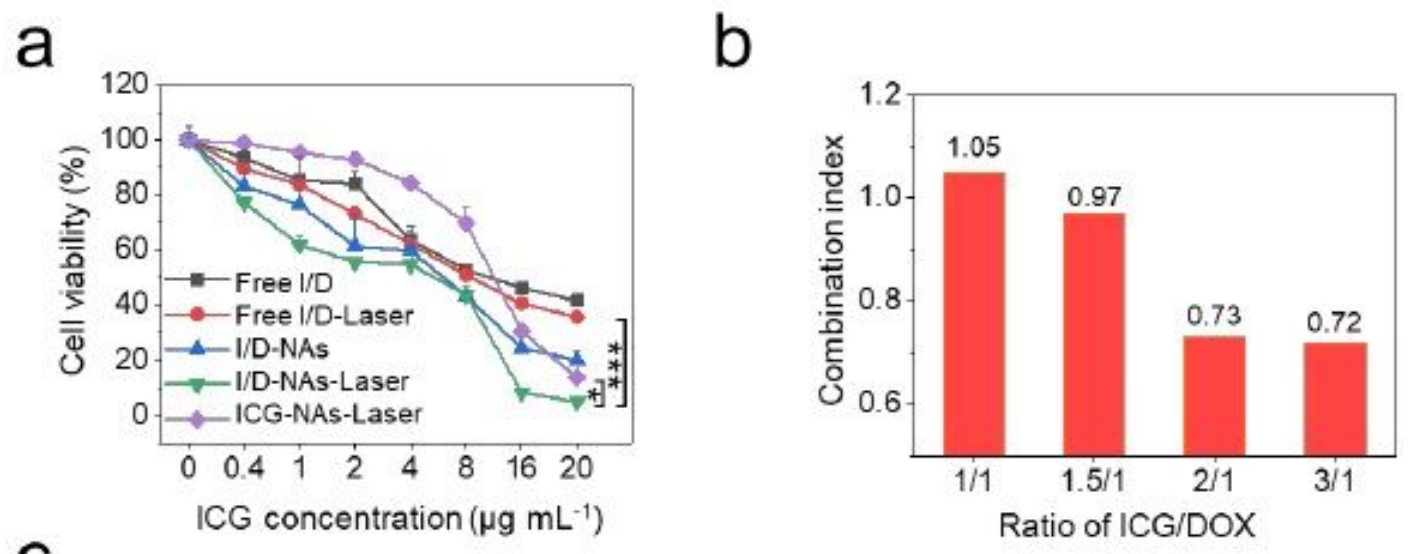

C
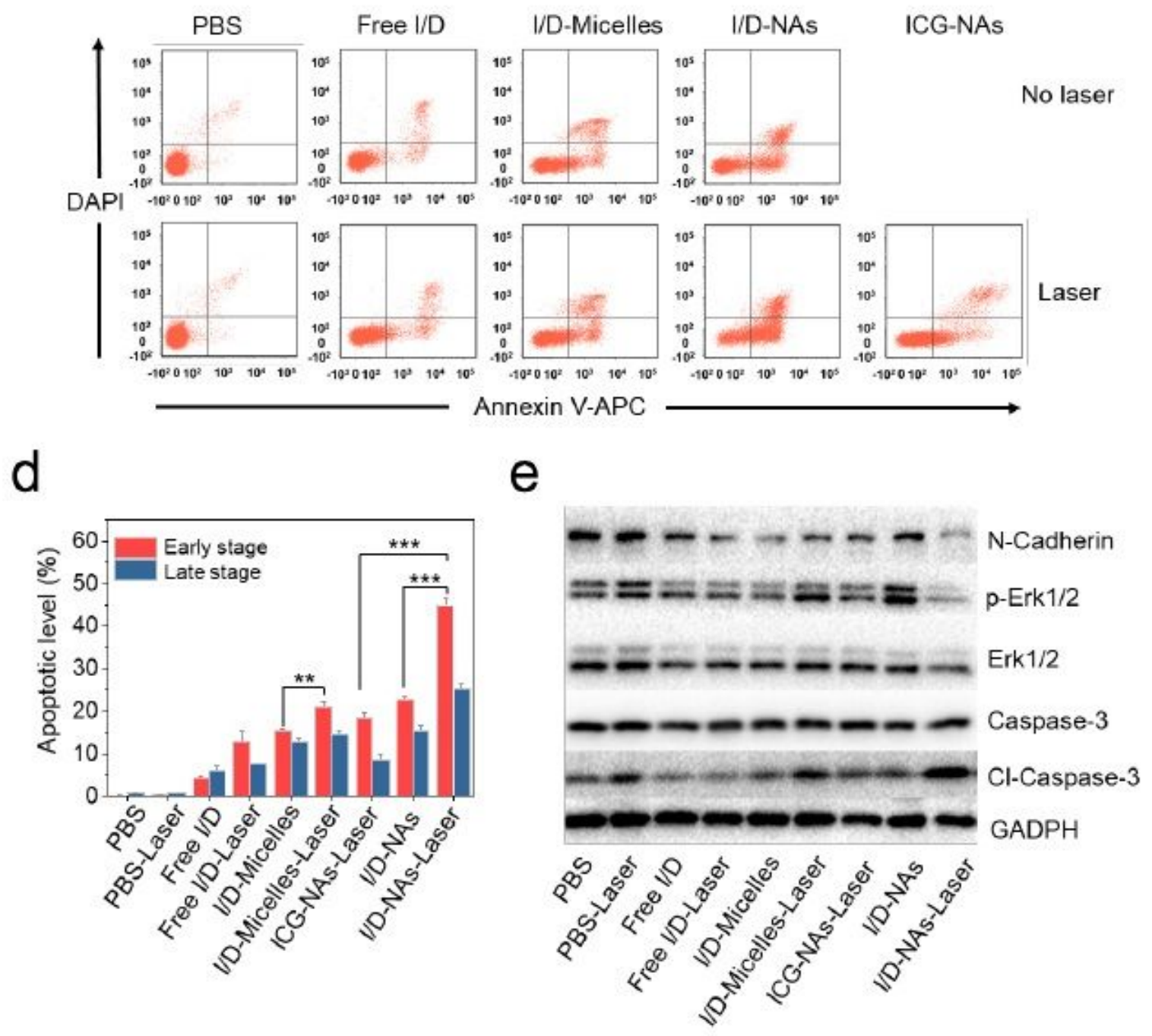


\section{Figure 4}

Cooperative cytotoxicity, antitumour and antimetastatic mechanisms of I/D-NAs. a. Cell viability of 4T1 cells treated with I/D-NAs and Free I/D under $1.5 \mathrm{~W} \mathrm{~cm}-2$ irradiation (785 nm, $3 \mathrm{~min}$ ) or not. b. Combination index of I/D-NAs at various ratios of ICG:DOX. c. Apoptotic levels of 4T1 cells stained by Annexin V-APC/DAPI Apoptosis Detection $761 \mathrm{Kit}$ after $24 \mathrm{~h}$ incubation with PBS, Free I/D, I/D-Micelles and I/D-NAs at the dose of $10.0 \mu \mathrm{g} \mathrm{mL}-1$ ICG under 3 min irradiation $(785 \mathrm{~nm}, 1.5 \mathrm{~W} \mathrm{~cm}-2)$ or not and (d) their apoptotic percentages of early stage and late stage. e. Western blotting of HSP 70, N-cad, p-ERK and Cl-caspase-3 levels at $6 \mathrm{~h}$ post-irradiation in 4T1 cells after incubation with PBS, Free I/D, I/D-Micelles and I/D-NAs at the dose of $10.0 \mu \mathrm{g} \mathrm{mL}-1$ ICG under 3 min irradiation $(785 \mathrm{~nm}, 1.5 \mathrm{~W} \mathrm{~cm}-2)$ or not. Statistical analysis was performed using two-sided student's t-test, ${ }^{*} p<0.05$, ${ }^{* *} p<0.01$, and ${ }^{* * *} p<0.001$. 
a

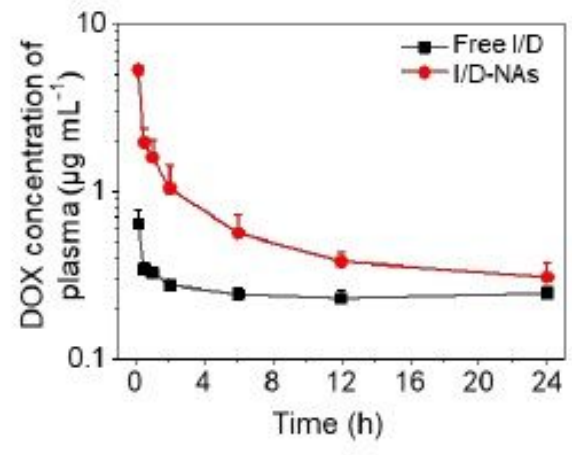

C

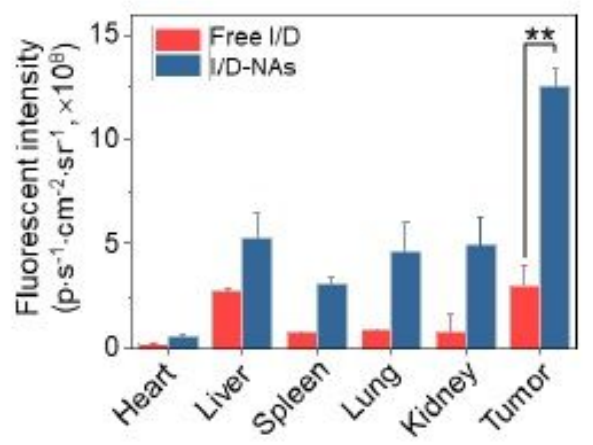

e

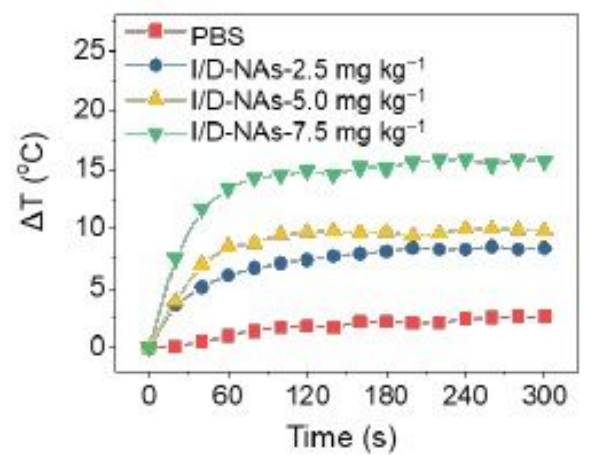

g

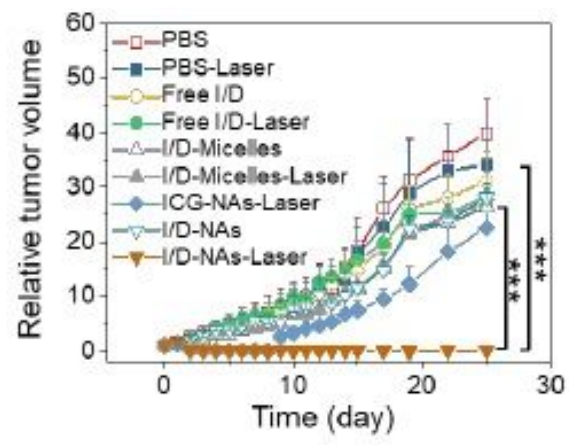

b

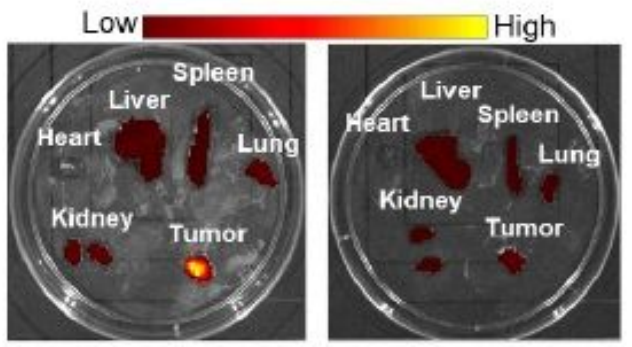

I/D-NAs

Free 1/D

d

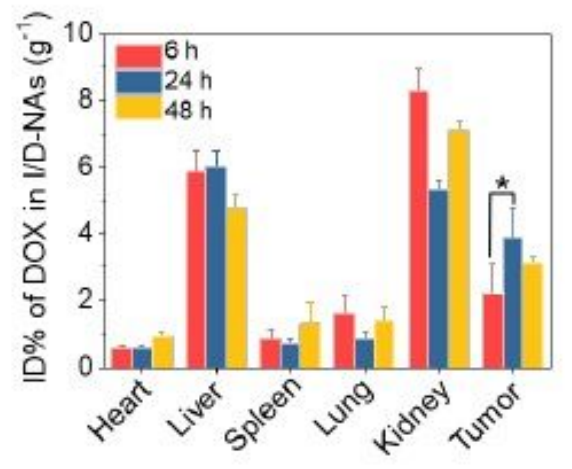

DHE
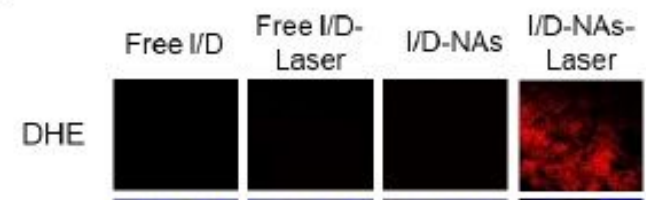

Hoechst

33342
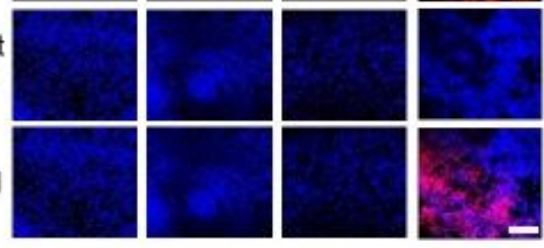

$\mathrm{h}$

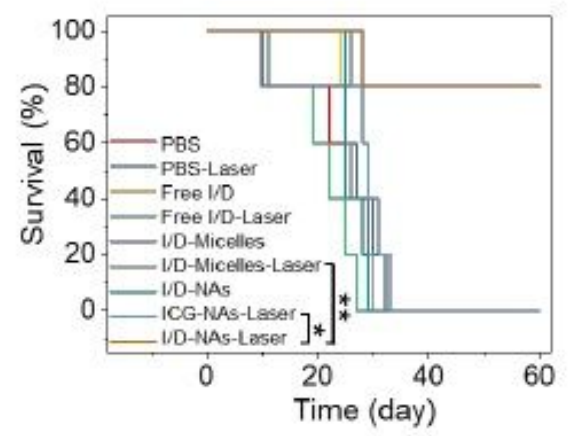

\section{Figure 5}

Photochemotherapeutic synergy of I/D-NAs against subcutaneous TNBC tumour models caused by multi-stimuli responsiveness. a. DOX concentrations in plasma from Free I/Dand I/D-NAs at the dose of $7.5 \mathrm{mg} \mathrm{kg}-1$ ICG. b. Ex vivo NIRF images of heart, 774 liver, spleen, lung, kidney, and tumour extracted from the mice bearing 4T1 cells treated with Free I/D and I/D-NAs at the dose of $7.5 \mathrm{mg} \mathrm{kg}-1$ ICG and (c) their NIRF intensities. d. Biodistribution of DOX in heart, liver, spleen, lung, kidney, and tumour of the mice 
bearing 4T1 subcutaneous tumours treated with I/D-NAs at different time post-injection at the dose of 7.5 mg kg-1 ICG. e. Temperature elevations of the mice bearing 4T1 cells treated with I/D-NAs at different doses of ICG under $785 \mathrm{~nm}$ irradiation at $1.5 \mathrm{~W} \mathrm{~cm}-2$. f. DHE staining of tumour sections treated with Free I/D and I/D-NAs at the dose of $7.5 \mathrm{mg} \mathrm{kg}-1$ ICG under $3 \mathrm{~min}$ irradiation or not (scale bar: $100 \mu \mathrm{m}$ ). $\mathrm{g}$.

Tumour growth profiles of the mice bearing 4T1 subcutaneous tumours with various treatments at the dose of $7.5 \mathrm{mg} \mathrm{kg}-1$ ICG, and (h) their Kaplan-Meier survival plots of the mice. The statistical analysis was determined by two-sided student's t-test, ${ }^{*} \mathrm{p}<0.01$ and ${ }^{* *} \mathrm{p}<0.001$. The statistical analysis was carried out using two-sided log-rank (Mantel-Cox) test for $h,{ }^{\star} p<0.05$ and ${ }^{*} \mathrm{p}<0.01$. 


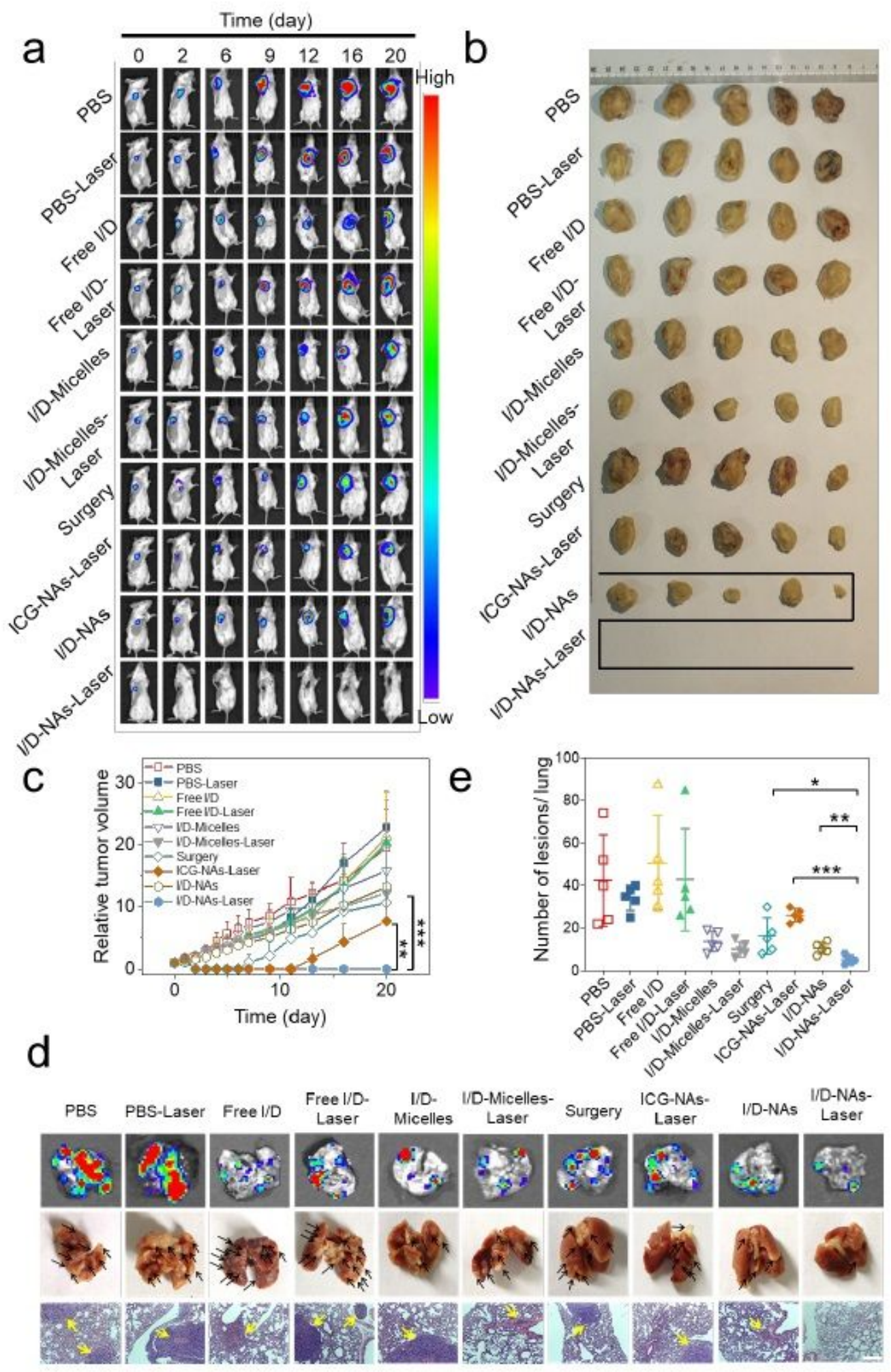

\section{Figure 6}

Photochemotherapeutic synergy of I/D-NAs against orthotopic and metastatic TNBC tumour models. a. In vivo bioluminescence images to track the tumour growth of the mice bearing orthotopic 4T1 breast tumours after various treatments. b. The photographs 790 of tumours extracted from the mice at 20 days post-injection as indicated in a. c. Tumour growth profiles of mice with various treatments as indicated in a. d. Exo vivo bioluminescence images, optical photographs and H\&E staining images (scale bar: 200 
$\mu \mathrm{m})$ of the lungs from the mice at 20 days post-injection as indicated in a. e. Counts of metastatic nodules in the lungs of the mice at 20 days post-injection as indicated in a. Statistical analysis was determined by two-sided student's t-test, ${ }^{\star} p<0.05,{ }^{* \star} \mathrm{p}<0.01$ and ${ }^{* \star *} \mathrm{p}<0.001$. 